\title{
Multi-modality imaging for treatment response evaluation in rectal cancer
}

Citation for published version (APA):

Janssen, M. H. (2011). Multi-modality imaging for treatment response evaluation in rectal cancer. [Doctoral Thesis, Maastricht University]. Datawyse / Universitaire Pers Maastricht. https://doi.org/10.26481/dis.20110914mj

Document status and date:

Published: 01/01/2011

DOI:

10.26481/dis.20110914mj

Document Version:

Publisher's PDF, also known as Version of record

\section{Please check the document version of this publication:}

- A submitted manuscript is the version of the article upon submission and before peer-review. There can be important differences between the submitted version and the official published version of record.

People interested in the research are advised to contact the author for the final version of the publication, or visit the DOI to the publisher's website.

- The final author version and the galley proof are versions of the publication after peer review.

- The final published version features the final layout of the paper including the volume, issue and page numbers.

Link to publication

\footnotetext{
General rights rights.

- You may freely distribute the URL identifying the publication in the public portal. please follow below link for the End User Agreement:

www.umlib.nl/taverne-license

Take down policy

If you believe that this document breaches copyright please contact us at:

repository@maastrichtuniversity.nl

providing details and we will investigate your claim.
}

Copyright and moral rights for the publications made accessible in the public portal are retained by the authors and/or other copyright owners and it is a condition of accessing publications that users recognise and abide by the legal requirements associated with these

- Users may download and print one copy of any publication from the public portal for the purpose of private study or research.

- You may not further distribute the material or use it for any profit-making activity or commercial gain

If the publication is distributed under the terms of Article $25 \mathrm{fa}$ of the Dutch Copyright Act, indicated by the "Taverne" license above, 


\section{Multi-modality imaging for treatment response evaluation in rectal cancer}




\section{Cover}

FDG-PET-CT images of a rectal cancer patient before, during and after pre-operative treatment with chemoradiotherapy. From left to right: prior to the start of treatment, one and two weeks after the onset of preoperative treatment and prior to surgery. From the FDG-PET-CT images, tumor shrinkage can be observed. As can be seen from the FDG-PET-CT images performed prior to surgery, no PET-positive residual tumor volume is present. Therefore, this patient was classified as a metabolic complete responder.

\section{Production}

Printing: Datawyse | Universitaire Pers Maastricht

ISBN 9789461590756

(C) Copyright M.H.M. Janssen, Maastricht 2011

The work presented in this thesis was made possible by technical and financial support of Siemens MI as well as by financial support from the Netherlands Organization for Health Research and Development (ZonMW; fellowship awarded to Guido Lammering). 


\title{
Multi-modality imaging for treatment response evaluation in rectal cancer
}

\author{
Proefschrift \\ ter verkrijging van de graad van doctor aan de Universiteit Maastricht, op gezag van de \\ Rector Magnificus prof. mr. G.P.M.F. Mols, volgens het besluit van het College van \\ Decanen, in het openbaar te verdedigen op woensdag 14 september 2011 door
}

Marco Henricus Maria Janssen

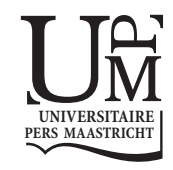




\section{Promotor}

Prof. dr. P. Lambin

\section{Copromotoren}

Dr. G. Lammering

Dr. M. Öllers

\section{Beoordelingscommissie}

Prof. dr. F. Mottaghy (voorzitter)

Prof. dr. R. Beets-Tan

Dr. R. Boellaard

Prof. dr. K. Haustermans 


\section{Contents}

Introduction

Chapter 1 Introduction and outline of the thesis

\section{Part I: FDG-PET-CT based treatment response evaluation and prediction}

Chapter 2 Evaluation of early metabolic responses in rectal cancer during combined chemo-radiotherapy or radiotherapy alone: sequen tial FDG-PET-CT findings

Chapter 3 Accurate prediction of pathological rectal tumor response after 2 weeks of pre-operative chemo-radiotherapy using FDG-PET-

CT imaging

Chapter 4 Blood glucose level normalization and accurate timing im proves the accuracy of PET-based treatment response predict tions in rectal cancer

Chapter 5 PET-based treatment response evaluation in rectal cancer: prediction and validation

\section{Part II: Perfusion-CT imaging during short course hypofractionated} radiotherapy

Chapter 6 Tumor perfusion increases during hypofractionated short course radiotherapy in rectal cancer: sequential perfusion-CT findings

Chapter 7 Repeated PET-CT and perfusion-CT imaging in rectal cancer: FDG uptake corresponds with tumor perfusion

\section{Part III: Validation of automatic PET-based tumor delineation}

Chapter 8 Volumetric pathological validation of FDG-PET based automatic tumor delineation of rectal cancer

\section{Discussion}

Chapter 9 General discussion and future perspectives

Summary

Samenvatting

Dankwoord

Curriculum Vitae

Publications 



\section{Introduction}





\section{Chapter}

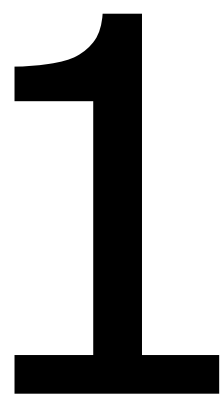

Introduction and outline of the thesis 


\subsection{Rectal cancer: epidemiology \& prognosis}

Rectal cancer is the malignant tumor growth from the inner wall of the final part of the large intestine. Risk factors for rectal cancer include heredity, polyps and chronic ulcerative colitis. Rectal cancer can be divided into two categories: non-locally advanced rectal cancer (NLARC) and locally advanced rectal cancer (LARC). For the patients with a clear margin between the tumor and the mesorectal fascia (MRF), observed from pre-treatment magnetic resonance (MR) images, the tumor is classified as NLARC, whereas if from the MR-images an invasion of the tumor into the MRF or involvement of the tumor into regional lymph nodes is observed, the tumor is classified as LARC.

According to the TNM classification, rectal cancer is divided into four stages on basis of the anatomical extent of the tumor ${ }^{1}$. The TNM classification is used to define the extend of disease with respect to the primary tumor $(T)$, involvement of regional lymph nodes $(\mathrm{N})$ and the presence of distant metastases (M) ${ }^{1}$.

\section{$\underline{T-P r i m a r y}$ Tumor}

T1 Tumor invades submucosa

T2 Tumor invades muscularis submucosa

T3 Tumor invades subserosa or into non-peritonealized pericolic or perirectal tissue

T4 Tumor directly invades other organs or structures and/or perforates visceral peritoneum

\section{$\underline{N-R e g i o n a l ~ L y m p h ~ N o d e s ~}$}

N0 No regional lymph node metastasis

N1 Metastasis in 1-3 regional lymph nodes

N2 Metastasis in 4 or more regional lymph nodes

\section{M-Distant Metastasis}

M0 No distant metastasis

M1 Distant metastasis

Within the Netherlands, the incidence and prevalence of rectal cancer increased over the last 10 years (Table 1.1) ${ }^{2}$. The prognosis of rectal cancer is related to the degree of penetration of the tumor through the rectal wall, the possible involvement of the tumor into regional lymph nodes and the presence of distant metastasis ${ }^{2}$. Also, the circumferential resection margin (CRM) after surgery is prognostic for locoregional tumor control ${ }^{3}$. A positive CRM, with the CRM involved by tumor or a distance of less than $1 \mathrm{~mm}$ between tumor and the CRM, is an important predictor of local and distant recurrence and patient survival ${ }^{3}$. The absolute number of rectal cancer related deaths slightly increased over the last 10 years ${ }^{2}$. 
However, taking into account the strong increase of the incidence and prevalence of rectal cancer, the relative number of rectal cancer related deaths decreased over the last decade. Also, the 5 year survival probability significantly increased over the last 30 years due to changes and standardization of the surgical approach and pre- and/or post-operative treatment with chemo- and radiotherapy (Table 1.1) ${ }^{2}$.

Table 1.1: Overview of the incidence, prevalence, number of related deaths and 5 year survival probability of rectal cancer within the Netherlands.

\begin{tabular}{||c|c|c|c|c||}
\cline { 3 - 5 } \multicolumn{1}{c|}{} & $\mathbf{2 0 0 0}$ & $\mathbf{2 0 0 5}$ & $\mathbf{2 0 1 0}$ \\
\hline \hline Incidence & Male & 1848 & 2206 & 2633 \\
\hline \hline & Female & 1292 & 1438 & 1543 \\
\hline \hline Prevalence & Male & 10874 & 13713 & 17049 \\
\hline \hline & Female & 8428 & 9860 & 11274 \\
\hline \hline & Male & 495 & 542 & 577 \\
\hline & Female & 390 & 398 & 387 \\
\hline
\end{tabular}

\begin{tabular}{|c|c|c|c|c|c||}
\cline { 3 - 6 } \multicolumn{2}{c|}{} & 1975 & 1985 & 1995 & $\mathbf{2 0 0 0}$ \\
\hline \multirow{2}{*}{5 year survival } & Male & $39 \%$ & $41 \%$ & $57 \%$ & $58 \%$ \\
\hline & Female & $41 \%$ & $42 \%$ & $50 \%$ & $58 \%$ \\
\hline
\end{tabular}

\subsection{Treatment Modalities}

For the treatment of rectal cancer, three treatment modalities can be used: radiotherapy, chemotherapy and surgery. Each of the treatment modalities can be used alone, but often, modalities are combined based on patient and tumor characteristics.

\section{Radiotherapy}

Radiotherapy (RT) is the medical use of ionizing radiation to treat malignancies. The patient referred to RT treatment is exposed to highly energetic megavoltage photon beams generated with a linear accelerator. With dedicated treatment planning software, the shape and direction of the treatment beams are positioned in such a way that they result in a sufficient dose to the tumor while limiting the radiation burden to the healthy tissue of organs surrounding the tumor. For patients diagnosed with rectal cancer, RT alone or in combination with chemotherapy, is most often the treatment of choice. In Maastricht, the Netherlands, patients diagnosed with NLARC are treated with hypofractionated RT ( 5 fractions of 5 Gy on 5 consecutive working days), followed by surgery. For NLARC, pre-operative RT is primarily used to lower the risk of local failure ${ }^{4-7}$. More advanced rectal tumors (LARC), with invasion of the tumor into the mesorectal fascia or involvement of the tumor in regional lymph nodes, are treated with long course RT ( 28 fractions, each of $1.8 \mathrm{~Gy}$ ) combined with chemotherapy. Preoperative treatment with long course combined chemo-radiotherapy (CRT) results in significant downsizing, downstaging and regression of the primary tumor ${ }^{8-12}$. 


\section{Chemotherapy}

Chemotherapy refers to the treatment of cancer with antineoplastic drugs by impairing the (mitotic part of the) cell cycle. The antineoplastic drugs kill cells that divide rapidly, which is one of the main properties of malignant cells. However, chemotherapy treatment also affects non-malignant cells that divide rapidly, for example cells in the bone marrow, the digestive tract and hair follicles. In Maastricht, The Netherlands, patients diagnosed with LARC are most often treated with long course RT (28 fractions of 1.8 Gy) combined with concomitant chemotherapy. In Europe, 5-fluorouracil (5-FU) is used for chemotherapy treatment of rectal cancer. 5-FU irreversible inhibits the enzyme thymidylate synthetase, ultimately blocking the synthesis of pyrimidine thymidine, a nucleotide required for DNA replication ${ }^{13}$. For the patients included in the studies within this thesis, capecitabine (Xeloda) was orally administered to the patients as a chemotherapeutic agent. Capecitabine is a pro-drug of 5-FU, which is enzymatically converted to $5-\mathrm{FU}^{13}$.

\section{Surgery}

Nowadays, for patients diagnosed with rectal cancer, surgery is the major component of treatment. However, conventional surgery, without pre-operative treatment with chemo- and/or radiotherapy, resulted in a high probability of local recurrence (average: $22.5 \%$, range: $9-36 \%)^{14}$. Over the last years, in Europe, the total mesorectal excision (TME) has been standardized for the treatment of rectal cancer. The introduction of the standardized TME surgical approach has led to a significant decrease of the number of local recurrences and an increased probability of disease free survival when compared to the conventional, non-standardized surgical approach used prior to the introduction of the $\mathrm{TME}^{7,15}$. Also, training of the surgeons performing the TME has been proven to result in an improved outcome for the patients ${ }^{16,17}$. However, limiting the performance of rectal cancer surgery to highly specialized surgeons or to only those surgeons who perform more than a certain volume is impractical in view of the prevalence of rectal cancer in Western Europe ${ }^{17}$.

Pre-operative treatment with short course hypofractionated RT, followed by a TME was found to decrease the probability of local recurrence even more, whereas pre-operative treatment with long course CRT resulted in a significant downsizing and downstaging of the primary tumor ${ }^{5,7-12}$. In $15-30 \%$ of the patients even complete regression of the primary tumor was observed after pre-operative treatment with CRT.

In Maastricht, The Netherlands, pre-operative treatment with either short course hypofractionated RT (NLARC) or combined long course CRT (LARC) is always followed by a TME. However, performing a TME is rather invasive with a significant risk on complications. For patients with relatively low seated tumors, a TME could result in loss of the sphincter, which would have serious negative impact on the quality of life for the patient. Patients with a strong or even complete pathological response to preoperative treatment could be referred to a less invasive surgical approach or even delayed or cancelled surgery. For patients with a small residual tumor volume with no tumor invasion in the MRF or involvement of the tumor into the regional lymph nodes, a less invasive surgical approach like a transanal endoscopic microsurgical approach could be used ${ }^{18}$. It would therefore be desirable to differentiate pathological responding from non-responding patients as early as possible during pre-operative treatment. 
Within this thesis, we tried to differentiate pathological responders from nonresponders during/after pre-operative treatment with the help of advanced multimodality medical imaging.

\subsection{Medical Imaging Modalities}

Prior to cancer treatment, (multi-modality) medical imaging is often used to obtain information about the location, size and stage of the malignancy. Anatomical imaging (computed tomography (CT) or magnetic resonance imaging (MRI)), provides insight in the location, size and stage of the tumor, whereas functional imaging with positron emission tomography (PET), dynamic contrast enhanced MRI (DCE-MRI) or perfusion$C T(p C T)$, gives insight in functional processes within the imaged malignancy. Functional imaging is used to measure or quantify changes in the metabolism, blood flow or chemical composition of the imaged tissue by studying the spatial distribution of an administered tracer or contrast agent. Within radation oncology, medical images are used for pre-treatment staging of the tumor and for radiotherapy treatment planning. However, over the last years, medical imaging, especially functional imaging, has been used more and more to evaluate treatment related responses during and after preoperative treatment.

\section{Computed Tomography}

Computed tomography (CT) is a medical imaging modality which generates a three dimensional (3D) image of an object from a series of two dimensional (2D) crosssectional $\mathrm{X}$-ray images using ionizing radiation. The $\mathrm{X}$-ray images are generated using an X-ray source that rotates around the object and an array of detectors positioned on the opposite side of the X-ray source. Within the field of radiation oncology, CT-images are used to plan the highly energetic photon beams of a linear accelerator to treat the patient with radiotherapy, ensuring sufficient radiation dose to the tumor while limiting the radiation burden to the surrounding healthy tissues and possible organs at risk.

\section{Magnetic Resonance Imaging}

Magnetic Resonance Imaging (MRI) makes use of magnetism and radio waves to produce 3D images of an imaged object. A circular magnet placed around the scanned object creates a strong magnetic field which aligns the protons of hydrogen atoms within the object. Exposure of the scanned object to radio waves spins the protons within the scanned object, producing a signal that is detected by a receiver coil of the MRI scanner. Relative to CT-imaging, MRI provides with a good spatial resolution and a good contrast between different soft tissues within the human body. MR-imaging makes no use of ionizing radiation, whereas for CT-imaging an X-ray source is used exposing ionizing radiation.

\section{Positron Emission Tomography}

Positron emission tomography (PET) is a medical imaging modality which produces a $3 D$ representation of functional processes within a scanned object. For this imaging modality, a positron emitting radionuclide is injected into the bloodstream of the 
patient. The emitted positron interacts with an electron, producing a pair of annihilation photons of $511 \mathrm{keV}$ moving in opposite directions. The photons are detected by the scintillator crystal detectors of the PET-scanner. In oncology, the most common tracer for PET-imaging is fluorodeoxyglucose (FDG), a glucose analog with the positron emitting radioactive isotope fluorine-18 $\left[{ }^{18} \mathrm{~F}\right]$ substituted for the normal hydroxyl group at the $2^{\prime}$ position of the glucose molecule. Similar to glucose, FDG is avidly consumed by cells in the brain, kidney and cancer cells. After uptake within the cell, phosphorylation of the FDG by hexokinase prevents it from being released again from the cell ${ }^{19}$. Furthermore, the fluorine atom prevents it from further metabolization, and hereby, FDG is metabolically trapped inside the cell ${ }^{19}$.

\section{Dynamic Contrast Enhanced Magnetic Resonance Imaging}

Dynamic contrast enhanced magnetic resonance imaging (DCE-MRI) makes use of repeated MR-imaging to evaluate and quantify the distribution of a contrast agent over time. An intravenously injected contrast agent circulates through the body and diffuses over time from the bloodstream into the extravascular and extracellular space. As the concentration of the administered contrast agent within the tissue increases, the signal intensity of the voxel within the MR-image representing this tissue also increases. A two compartment kinetic model (Kety-model) can be used to quantify tissue perfusion based on the measured relative signal changes ${ }^{20}$.

\section{Perfusion-CT Imaging}

As DCE-MRI, perfusion-CT (pCT) imaging is a method of dynamic imaging by which perfusion of a tissue is measured, studying the distribution of an intravenously administered (iodinated) contrast agent over time. From the contrast uptake curves of a blood vessel and the studied tumor tissue, tumor perfusion can be quantified using kinetic modeling techniques.

For primary staging of malignancies, MRI was presented as the most accurate imaging modality for the determination of the margin between the tumor and the mesorectal fascia, whereas this margin could not be accurately determined from FDG-PET images $12,21,22$. Also, MRI generally presents better soft-tissue contrast compared to CT imaging. For the evaluation of the lymph node status of the patient, MR imaging is superior to CT and PET imaging because of the the high spatial resolution and soft-tissue contrast of MRI. Contrast enhanced MR-imaging even significantly improves the sensitivity and specificity of nodal staging and restaging in rectal cancer when compared to standard MRI ${ }^{23}$. Therefore, in Maastricht, The Netherlands, MRI is used for pimary staging of rectal tumors prior to treatment.

For the detection of distant metastases and the evaluation of tumor response during or after treatment, functional imaging with FDG-PET-imaging is most often used. Repeated FDG-PET-imaging at multiple time-points during and/or after treatment was found to result in an accurate prediction of the pathological treatment response ${ }^{10-12 \text {, }}$ 24-35. A prediction of the pathological treatment response of a malignancy already early during pre-operative treatment enables modifications of the treatment and/or planned surgical approach. 
Also, the FDG uptake within malignancies, assessed with PET-imaging, can be used to automatically delineate the tumor using standardized uptake value (SUV) thresholding 36. Automatic tumor contouring is largely user independent, whereas manual tumor delineation is subject to the clinical interpretation of the medical images by the radiation oncologist delineating the tumor ${ }^{37-39}$.

However, there are two major limitations to the use of FDG-PET imaging in the treatment of cancer patients. First, a non-tumor specific increased FDG uptake is observed for different organs, for example kidney, liver, brain and bladder. Also, (peritumoral) inflammatory reactions, due to anti-cancer treatment, show an enhanced FDG uptake ${ }^{40,41}$. When the tumor is located within or in the direct neighborhood of an organ presenting with non-tumor specific increased FDG uptake or the patient presents with a peritumoral inflammatory response, it is difficult to quantify the FDG uptake level within the tumor.

The second drawback of FDG-PET-imaging is the limited spatial resolution of the current available PET-scanners. Accurate and reliable imaging of small malignancies is still difficult due to the partial volume effect (PVE) ${ }^{42}$. The PVE can result in an underestimation of the FDG uptake within the imaged tissues. For most patients preoperatively treated with CRT, the tumor volume decreases over time as an effect of cancer-treatment. Based on FDG-PET-imaging, it is very difficult to conclude whether there is residual (metabolic active) malignant tissue present after pre-operative treatment for patients with a strong (metabolic) treatment response. Besides these two drawbacks, it is known that when quantifying the FDG uptake within malignancies from sequentially performed PET-images or PET-images coming from different hospitals, standardization of the imaging-protocol is required ${ }^{43-45}$. This, since the FDG uptake within tumors is known to be dependent on many factors, for example the blood glucose level (BGL) of the patient at the time of PET-imaging, the FDG uptake period as well as reconstruction parameters ${ }^{45-53}$.

For the non-invasive evaluation of tissue perfusion, functional imaging with DCE-MRI is most often used ${ }^{25,54,55}$. However, it has been shown that PCT imaging is comparable to DCE-MRI for the quantification of perfusion of rectal tumors ${ }^{56}$. The pre-treatment perfusion level as well as changes in tumor perfusion during of after (chemo-) radiotherapy treatment, assessed with either CT or MR imaging, have successfully been correlated to the pathological treatment response ${ }^{25,57-64}$. In radiotherapy departments, the access to MR-scanners is limited compared to the availability of CTscanners ${ }^{56}$. Also, pCT scans can be performed directly after the (PET-)CT scan performed for treatment planning on the same CT-scanner, ensuring an identical patient orientation for both scans. $\mathrm{pCT}$ imaging results in an additional radiation burden for the patient, whereas DCE-MRI makes does not make use of ionizing radiation ${ }^{65}$. However, the additional radiation dose due to a pCT-scan is rather low compared to the total radiation dose of the $\mathrm{RT}$ treatment. 


\subsection{Objectives and outline of the thesis}

The main objective of this thesis was to study treatment related changes in the FDG uptake within and perfusion of rectal tumors during pre-operative treatment with RT alone or combined CRT and to investigate the correlation between changes in these functional processes within the tumor and the pathological treatment response of the tumor. An accurate prediction of the pathological treatment response early during preoperative treatment would enable response guided modifications of the treatment protocol, which could ultimately result in individualized (chemo-)radiotherapy and surgical approaches.

\section{Part I: FDG-PET-CT based treatment response evaluation and prediction}

In Chapter $\mathbf{2}$ we investigated the early effects of hypofractionated RT and combined CRT on the metabolic activity of rectal tumors. The metabolic treatment response to either short course hypofractionated RT or long course RT combined with concomitant chemotherapy was studied using sequential FDG-PET-CT imaging.

The metabolic treatment responses of rectal tumors pre-operatively treated with CRT were correlated to the pathological treatment responses in Chapter $\mathbf{3}$ of this thesis. An accurate prediction of the pathological treatment response already early during preoperative treatment would ultimately enable PET-based modifications of the treatment protocol.

Quantification of PET-scans is known to be dependent upon the imaging and reconstruction protocol as well as on fluctuations in the patient conditions. Therefore, in Chapter 4, the time sensitivity of FDG uptake within rectal tumors was studied during the first hours after FDG injection. Also, the influence of fluctuations in the blood glucose level of the patient on the accuracy of PET-based response predictions was investigated.

To prove the applicability of PET-based predictions of the pathological treatment response early during pre-operative treatment for patients not included in the patient group used to develop the predictive model, the PET-based predictive model presented in Chapter 3 was applied to a group of newly included patients in Chapter $\mathbf{5}$ of this thesis.

\section{Part II: Perfusion-CT imaging during short course hypofractionated radiotherapy}

Since no metabolic treatment response was found in Chapter 2 for the patients treated with short course hypofractionated RT, in Chapter 6 of this thesis, tumor perfusion was studied prior to and after pre-operative treatment with hypofractionated RT to investigate possible early treatment related changes of tumor perfusion.

In Chapter 7, quantification of the FDG uptake within rectal tumors was correlated to tumor perfusion assessed with perfusion-CT imaging to study the correspondence between FDG uptake and tumor perfusion on both inter- and intra-tumor level. 


\section{Part III: Validation of automatic PET-based tumor delineation}

For modern RT techniques, an accurate delineation of the tumor volume is required. Since manual tumor delineation on medical images is known to be subject to clinical interpretation, there is a growing interest in automatic techniques for tumor contouring. In Chapter 8 of this thesis, the volume of the tumor contour resulting from SUV thresholding was validated with pathology for patients diagnosed with NLARC.

In Chapter 9, a summary and a general discussion with future perspectives are presented. 


\subsection{References}

[1] Sobin L, G.M., Wittekind C., TNM Classification of malignant tumours, 7th edition. 7 ed. 2009 : Wiley-Blackwell.

[2] Signaleringscommissie kanker, Kanker in Nederland: trends, prognoses en implicaties voor zorgvraag. 2004: Drukkerij van den Bogaard, Oisterwijk B.V.

[3] Hermanek, P. and T. Junginger, The circumferential resection margin in rectal carcinoma surgery. Tech Coloproctol 2005;9:193-9; discussion 199-200.

[4] Improved survival with preoperative radiotherapy in resectable rectal cancer. Swedish Rectal Cancer Trial. N Engl J Med 1997;336:980-7.

[5] Kapiteijn, E., et al., Preoperative radiotherapy combined with total mesorectal excision for resectable rectal cancer. N Engl J Med 2001;345:638-46.

[6] Peeters, K.C., et al., The TME trial after a median follow-up of 6 years: increased local control but no survival benefit in irradiated patients with resectable rectal carcinoma. Ann Surg 2007;246:693-701.

[7] Peeters, K.C., E. Kapiteijn, and C.J. van de Velde, Managing rectal cancer: the Dutch experience. Colorectal Dis 2003;5:423-6.

[8] Valentini, V., et al., Ten years of preoperative chemoradiation for extraperitoneal T3 rectal cancer: acute toxicity, tumor response, and sphincter preservation in three consecutive studies. Int J Radiat Oncol Biol Phys 2001;51:371-83.

[9] Bosset, J.F., et al., Enhanced tumorocidal effect of chemotherapy with preoperative radiotherapy for rectal cancer: preliminary results--EORTC 22921. J Clin Oncol 2005;23:5620-7.

[10] Capirci, C., et al., Sequential FDG-PET/CT reliably predicts response of locally advanced rectal cancer to neo-adjuvant chemo-radiation therapy. Eur J Nucl Med Mol Imaging 2007;34:1583-93.

[11] Capirci, C., et al., Long-term prognostic value of 18F-FDG PET in patients with locally advanced rectal cancer previously treated with neoadjuvant radiochemotherapy. AJR Am J Roentgenol 2006;187:202-8.

[12] Vliegen, R.F., et al., Can an FDG-PET/CT predict tumor clearance of the mesorectal fascia after preoperative chemoradiation of locally advanced rectal cancer? Strahlenther Onkol 2008;184:457-64.

[13] Patel, P.A., Evolution of 5-fluorouracil-based chemoradiation in the management of rectal cancer. Anticancer Drugs 2011;22:311-6.

[14] Kapiteijn, E., et al., Local recurrence in patients with rectal cancer diagnosed between 1988 and 1992: a population-based study in the west Netherlands. Eur J Surg Oncol 1998;24:528-35.

[15] Kapiteijn, E. and C.J. van de Velde, The role of total mesorectal excision in the management of rectal cancer. Surg Clin North Am 2002;82:995-1007.

[16] Kapiteijn, E., H. Putter, and C.J. van de Velde, Impact of the introduction and training of total mesorectal excision on recurrence and survival in rectal cancer in The Netherlands. Br J Surg 2002;89:1142-9.

[17] Kapiteijn, E. and C.J. van de Velde, Developments and quality assurance in rectal cancer surgery. Eur J Cancer 2002;38:919-36.

[18] Paci, M., et al., [Transanal endocopic microsurgery (TEM) in advanced rectal cancer disease treatment]. Ann Ital Chir 2010;81:269-74; discussion 283.

[19] Phelps, M.E., PET Molecular Imaging and Its Biological Applications. 2003, New York: Springer.

[20] Tofts, P.S., et al., Estimating kinetic parameters from dynamic contrast-enhanced T(1)-weighted MRI of a diffusable tracer: standardized quantities and symbols. J Magn Reson Imaging 1999;10:223-32.

[21] Diagnostic accuracy of preoperative magnetic resonance imaging in predicting curative resection of rectal cancer: prospective observational study. BMJ 2006;333:779.

[22] Beets-Tan, R.G., et al., Accuracy of magnetic resonance imaging in prediction of tumour-free resection margin in rectal cancer surgery. Lancet 2001;357: 497-504.

[23] Lambregts, D.M., et al., Accuracy of gadofosveset-enhanced MRI for nodal staging and restaging in rectal cancer. Ann Surg 2011;253:539-45. 
[24] Capirci, C., et al., The role of dual-time combined 18-fluorodeoxyglucose positron emission tomography and computed tomography in the staging and restaging workup of locally advanced rectal cancer, treated with preoperative chemoradiation therapy and radical surgery. Int J Radiat Oncol Biol Phys 2009; 74:1461-9.

[25] Lambrecht, M., et al., The use of FDG-PET/CT and diffusion-weighted magnetic resonance imaging for response prediction before, during and after preoperative chemoradiotherapy for rectal cancer. Acta Oncol 2010;49:956-63.

[26] Mak, D., et al., The use of PET in assessing tumor response after neoadjuvant chemoradiation for rectal cancer. Radiother Oncol 2010;97:205-11.

[27] Kalff, V., et al., Findings on 18F-FDG PET scans after neoadjuvant chemoradiation provides prognostic stratification in patients with locally advanced rectal carcinoma subsequently treated by radical surgery. J Nucl Med 2006;47:14-22.

[28] Amthauer, H., et al., Response prediction by FDG-PET after neoadjuvant radiochemotherapy and combined regional hyperthermia of rectal cancer: correlation with endorectal ultrasound and histopathology. Eur J Nucl Med Mol Imaging 2004;31:811-9.

[29] Denecke, T., et al., Comparison of CT, MRI and FDG-PET in response prediction of patients with locally advanced rectal cancer after multimodal preoperative therapy: is there a benefit in using functional imaging? Eur Radiol 2005;15: 1658-66.

[30] Guillem, J.G., et al., Sequential preoperative fluorodeoxyglucose-positron emission tomography assessment of response to preoperative chemoradiation: a means for determining longterm outcomes of rectal cancer. J Am Coll Surg 2004;199:1-7.

[31] Melton, G.B., et al., Efficacy of preoperative combined 18-fluorodeoxyglucose positron emission tomography and computed tomography for assessing primary rectal cancer response to neoadjuvant therapy. J Gastrointest Surg 2007;11:961-9; discussion 969.

[32] Cascini, G.L., et al., 18F-FDG PET is an early predictor of pathologic tumor response to preoperative radiochemotherapy in locally advanced rectal cancer. J Nucl Med 2006;47:1241-8.

[33] Rosenberg, R., et al., The predictive value of metabolic response to preoperative radiochemotherapy in locally advanced rectal cancer measured by PET/CT. Int J Colorectal Dis 2009;24:191-200.

[34] Hindie, E., C. Hennequin, and J.L. Moretti, Predicting response to chemoradiotherapy in rectal and oesophageal cancer with 18F-FDG: prognostic value and possible role in patient management. Eur J Nucl Med Mol Imaging 2007; 34: 1576-82.

[35] de Geus-Oei, L.F., et al., Monitoring and predicting response to therapy with 18F-FDG PET in colorectal cancer: a systematic review. J Nucl Med 2009;50 Suppl 1:43S-54S.

[36] Daisne, J.F., et al., Tri-dimensional automatic segmentation of PET volumes based on measured source-to-background ratios: influence of reconstruction algorithms. Radiother Oncol 2003;69:247-50.

[37] van Baardwijk, A., et al., PET-CT-based auto-contouring in non-small-cell lung cancer correlates with pathology and reduces interobserver variability in the delineation of the primary tumor and involved nodal volumes. Int J Radiat Oncol Biol Phys 2007;68:771-8.

[38] Leunens, G., et al., Quality assessment of medical decision making in radiation oncology: variability in target volume delineation for brain tumours. Radiother Oncol 1993;29:169-75.

[39] Patel, D.A., et al., Impact of integrated PET/CT on variability of target volume delineation in rectal cancer. Technol Cancer Res Treat 2007;6:31-6.

[40] Kubota, R., et al., Intratumoral distribution of fluorine-18-fluorodeoxyglucose in vivo: high accumulation in macrophages and granulation tissues studied by microautoradiography. J Nucl Med 1992;33:1972-80.

[41] Larson, S.M., Cancer or inflammation? A Holy Grail for nuclear medicine. J Nucl Med 1994;35:1653-5.

[42] Soret, M., S.L. Bacharach, and I. Buvat, Partial-volume effect in PET tumor imaging. J Nucl Med 2007;48:932-45.

[43] Boellaard, R., et al., FDG PET and PET/CT: EANM procedure guidelines for tumour PET imaging: version 1.0. Eur J Nucl Med Mol Imaging 2010;37:181-200.

[44] Boellaard, R., et al., The Netherlands protocol for standardisation and quantification of FDG whole body PET studies in multi-centre trials. Eur J Nucl Med Mol Imaging 2008;35:2320-33. 
[45] Shankar, L.K., et al., Consensus recommendations for the use of 18F-FDG PET as an indicator of therapeutic response in patients in National Cancer Institute Trials. J Nucl Med 2006;47:1059-66.

[46] Beaulieu, S., et al., SUV varies with time after injection in (18)F-FDG PET of breast cancer: characterization and method to adjust for time differences. J Nucl Med 2003;44:1044-50.

[47] Keyes, J.W., Jr., SUV: standard uptake or silly useless value? J Nucl Med 1995; 36:1836-9.

[48] Visser, E.P., O.C. Boerman, and W.J. Oyen, SUV: from silly useless value to smart uptake value. J Nucl Med 2010;51:173-5.

[49] Vriens, D., et al., Methodological considerations in quantification of oncological FDG PET studies. Eur J Nucl Med Mol Imaging 2010;37:1408-25.

[50] Lindholm, P., et al., Influence of the blood glucose concentration on FDG uptake in cancer--a PET study. J Nucl Med 1993;34:1-6.

[51] Langen, K.J., et al., The influence of plasma glucose levels on fluorine-18-fluorodeoxyglucose uptake in bronchial carcinomas. J Nucl Med 1993;34:355-9.

[52] Adams, M.C., et al., A systematic review of the factors affecting accuracy of SUV measurements. AJR Am J Roentgenol 2010;195:310-20.

[53] Boellaard, R., Standards for PET image acquisition and quantitative data analysis. J Nucl Med 2009;50 Suppl 1:11S-20S.

[54] Goh, V., A.R. Padhani, and S. Rasheed, Functional imaging of colorectal cancer angiogenesis. Lancet Oncol 2007;8:245-55.

[55] Padhani, A.R., Functional MRI for anticancer therapy assessment. Eur J Cancer 2002;38:2116-27.

[56] Kierkels, R.G., et al., Comparison between perfusion computed tomography and dynamic contrast-enhanced magnetic resonance imaging in rectal cancer. Int J Radiat Oncol Biol Phys 2010;77:400-8.

[57] de Lussanet, Q.G., et al., Dynamic contrast-enhanced magnetic resonance imaging of radiation therapy-induced microcirculation changes in rectal cancer. Int J Radiat Oncol Biol Phys 2005;63:1309-15.

[58] Lambrecht, M., et al., Value of Diffusion-Weighted Magnetic Resonance Imaging for Prediction and Early Assessment of Response to Neoadjuvant Radiochemotherapy in Rectal Cancer: Preliminary Results. Int J Radiat Oncol Biol Phys, 2011.

[59] Lambregts, D.M., et al., Diffusion-Weighted MRI for Selection of Complete Responders After Chemoradiation for Locally Advanced Rectal Cancer: A Multicenter Study. Ann Surg Oncol, 2011.

[60] Sahani, D.V., et al., Assessing tumor perfusion and treatment response in rectal cancer with multisection CT: initial observations. Radiology 2005; 234: 785-92.

[61] Bellomi, M., et al., CT perfusion for the monitoring of neoadjuvant chemotherapy and radiation therapy in rectal carcinoma: initial experience. Radiology 2007;244:486-93.

[62] Park, M.S., et al., Perfusion CT: noninvasive surrogate marker for stratification of pancreatic cancer response to concurrent chemo- and radiation therapy. Radiology 2009;250:110-7.

[63] Wang, J., et al., Tumor response in patients with advanced non-small cell lung cancer: perfusion CT evaluation of chemotherapy and radiation therapy. AJR Am J Roentgenol 2009;193:1090-6.

[64] Zima, A., et al., Can pretreatment CT perfusion predict response of advanced squamous cell carcinoma of the upper aerodigestive tract treated with induction chemotherapy? AJNR Am J Neuroradiol 2007;28:328-34.

[65] Goh, V., et al., Radiation dose from volumetric helical perfusion CT of the thorax, abdomen or pelvis. Eur Radiol, 2010. 


\section{Part I:}

FDG-PET-CT based treatme nt response evaluation and prediction. 



\title{
Chapter
}

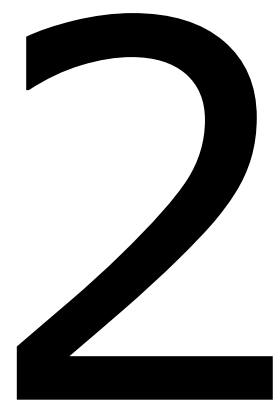

\section{Evaluation of early metabolic responses in rectal cancer during combined chemo-radiotherapy or radiotherapy alone: sequential FDG-PET-CT findings.}

\author{
Marco Janssen \\ Michel Öllers \\ Ruud van Stiphout \\ Jeroen Buijsen \\ Jørgen van den Bogaard \\ Dirk de Ruysscher \\ Philippe Lambin \\ Guido Lammering
}

Radiotherapy \& Oncology, 2010; 94(2): 151-155 


\section{Abstract \\ Purpose}

The purpose of this study was to prospectively investigate metabolic changes of rectal tumors after one week of treatment with either chemo-radiotherapy (28x1.8Gy + Capecitabine)(CRT) or hypofractionated radiotherapy (5x5Gy) alone (RT).

\section{Materials \& Methods}

Fourty-six rectal cancer patients, 25 CRT- and 21 RT-patients, were included in this study. Sequential FDG-PET-CT scans were performed for each of the included patients prior to treatment and after the first week of treatment. Consecutively, the metabolic treatment response of the tumor was evaluated.

\section{Results}

For the patients referred for pre-operative CRT, significant reductions of $\mathrm{SUV}_{\text {mean }}$ $(p<0.001)$ and SUV $_{\max }(p<0.001)$ within the tumor were found already after the first week of treatment ( $8 \mathrm{~Gy}$ biological equivalent dose (BED). In contrast, one week of treatment with RT alone did not result in significant changes in the metabolic activity of the tumor ( $p=0.767, p=0.434)$, despite the higher applied RT dose of 38.7Gy BED.

\section{Conclusions}

Chemo-radiotherapy of rectal cancer leads to significant early changes in the metabolic activity of the tumor, which was not the case early after hypofractionated radiotherapy alone, despite the higher radiotherapy dose given. This indicates that the chemotherapeutic agent Capecitabine might be responsible for the early metabolic treatment responses during chemo-radiotherapy in rectal cancer. 


\subsection{Introduction}

During the last years, sequential ${ }^{18} \mathrm{~F}$-fluorodeoxyglucose positron-emissiontomography computed tomography (FDG-PET-CT) imaging has been increasingly studied to monitor the metabolic response of the tumor to multimodality treatment of rectal cancer ${ }^{1-12}$. For rectal cancer, pre-operative short course radiotherapy (RT) was shown to result in improved local control, whereas pre-operative treatment with chemo-radiotherapy (CRT) was found to result in a significant downsizing and downstaging of the tumor, increasing the rate of complete surgical resection ${ }^{2-4,9,12}$. In 15$30 \%$ of the patients being pre-operatively treated with CRT, even complete tumor regression was observed 6 to 8 weeks after finishing the pre-operative treatment ${ }^{3,4,12 \text {, }}$ ${ }^{13}$. Over the years, many studies have been published reporting metabolic treatment response determinations of rectal carcinomas using dual time PET-imaging both before and after therapy, presenting a significant reduction of FDG uptake due to preoperative treatment with neo-adjuvant CRT ${ }^{1,3-7,10,12,14,15}$. However, in contrast to response evaluations based on PET-imaging before and after treatment, monitoring the tumor response early during pre-operative treatment enables response-guided modifications of the treatment protocol on the basis of early changes of FDG uptake, possibly strengthened by additional clinical or biological factors.

A significant reduction of the FDG uptake within rectal carcinomas was observed already after two weeks of pre-operative CRT, with the reduction of the FDG uptake being a good predictor of pathological treatment response ${ }^{2,8,11}$. However, not much is known about the possible cause of early changes in the metabolic activity of rectal tumors undergoing pre-operative CRT. Therefore, in order to better understand the early metabolic changes within the tumor during CRT, we thought it might be helpful to compare the metabolic response early during CRT with the metabolic response occurring after treatment with RT alone. This could lead to a better understanding of the biological basis for early changes and could help to improve the percentage of early responding tumors, thereby also possibly improving the prognosis of patients with rectal cancer. To our knowledge, very few direct comparative studies have been performed so far ${ }^{9}$. Thus, a prospective study was initiated to compare early metabolic treatment response in rectal cancer undergoing either concomitant CRT or RT alone.

\subsection{Materials \& Methods}

\section{Patient Characteristics}

Fourty-six patients diagnosed with rectal cancer were included in this study, from which the clinical TN staging was obtained from a pre-treatment MR-scan according to the TNM classification of malignant tumors (Edition 6). The included patients were treated according to the national and regional guidelines. According to these guidelines, patients with a T2N0-1 or a mid or high rectal T3NO-1 tumor with a predicted circumferential resection margin (CRM) on MR of $>2 \mathrm{~mm}$ were pre-operatively treated with short course hypofractionated RT ( 5 fractions of 5 Gy on consecutive working days). 
Patients with N2 disease, with low seated T3 tumors and patients with a tumor close to or invading the mesorectal fascia (CRM $<2 \mathrm{~mm}$ ) were treated with neoadjuvant CRT ( 28 fractions of $1.8 \mathrm{~Gy}$ daily; Capecitabine $825 \mathrm{mg} / \mathrm{m}^{2} \mathrm{BID}$ ). Twenty-five of the included patients were pre-operatively treated with CRT, whereas twenty-one patients were pre-operatively treated with RT alone. For the patients treated with hypofractionated short course RT, a TME was planned within 3 days after the last RT-fraction, whereas for the patients pre-operatively treated with CRT, the TME was planned approximately 3 months after the first RT fraction. The Medical Ethics Committee according to the Dutch law approved the trial and all patients gave written informed consent before entering the study.

\section{A: Chemoradiotherapy (CRT)}

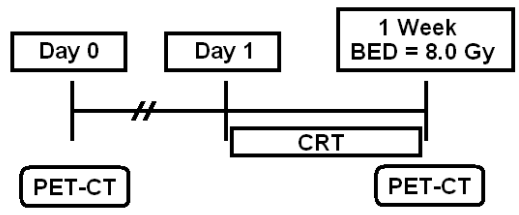

B: Radiotherapy (RT)

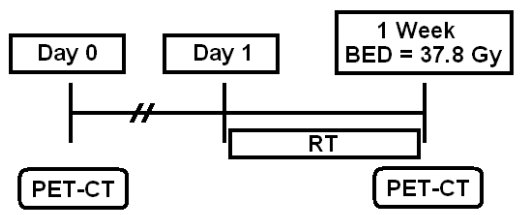

Figure 2.1: PET-CT study scheme for the assessment of the early metabolic treatment response during preoperative treatment of rectal cancer; A) study scheme for the patients treated with chemo-radiotherapy (CRT) B) study scheme for the patients treated with short course hypofractionated radiotherapy (RT).

\section{PET-CT Imaging and Processing}

Sequential FDG-PET-CT scans, both static and dynamic, were performed for each of the included patients at two different time points. The patients treated with CRT were imaged prior to CRT and one week after the onset of CRT (10.8 Gy, which corresponds to a biological effective dose (BED) of $8.0 \mathrm{~Gy}$ ) (Fig. $2.1 \mathrm{~A})^{16}$. Patients treated with RT were imaged prior to the start of RT and at the day of the fifth RT fraction (total dose of $25 \mathrm{~Gy}$, corresponding to a BED of $38.7 \mathrm{~Gy}$ ) (Fig. 2.1B) ${ }^{16}$. All patients were instructed to fast for at least 6 hours prior to FDG injection. All PET-CT scans were performed by use of a dedicated Siemens Biograph 40 TruePoint PET-CT scanner (Siemens Medical, Erlangen, Germany) with an axial field of $16.2 \mathrm{~cm}$, slice thickness of $3 \mathrm{~mm}$ and a pixel spacing of $5.3466 \mathrm{~mm}$ in both directions. The scanner is equipped with ulta-fast detector electronics (Pico 3D) and has a spatial resolution of approximately $6 \mathrm{~mm}$ full-widthat-half-maximum (FWHM). PET data acquisition was done 3 dimensional (3D), requiring a proper scatter correction. CT-based attenuation and decay correction was performed. PET-images were reconstructed from the acquired list-mode (LM) data using Fourier-rebinning(FORE) and ordered-subset-expectation-maximization-reconstruction (OSEM 2D) with 4 iterations and 8 subsets. The patient was positioned on a flat tabletop using a movable laser alignment system in a "head-first supine" position with the arms crossed above the chest, identical to the treatment position. For each scan, first a topogram was made from which the radiation oncologist manually selected the region of interest (ROI) for the dynamic PET-scan. Next, a CT-scan of the ROI was acquired, after which the FDG, with the activity depending on the weight of the patient (weight $\left.[\mathrm{kg}]^{*} 4+20[\mathrm{MBq}]\right)$, was injected intravenously followed by injection of physiologic- 
saline $(10 \mathrm{ml})$. Acquisition of the dynamic PET-scan over 60 minutes was started directly after FDG administration. The list-mode PET-data were rebinned and reconstructed using 28 time-frames (10x30 sec., $5 \times 60 \mathrm{sec}$., $5 \times 120 \mathrm{sec}$. and $8 \times 300 \mathrm{sec}$.) ${ }^{17}$. An additional static PET-CT scan, with an acquisition time of 5 minutes per bedposition, was acquired after finishing the dynamic PET-CT scan. Prior to each FDG injection, the patients blood glucose level (BGL) was measured using an automatic device (LifeScan One Touch Ultra, LifeScan Inc., Milpitas, USA). All acquired PET-data were normalized for the BGL using the following equation:

$S U V_{\text {normalized }}=S U V \cdot \frac{[G l u]}{100}$, with [Glu] the measured BGL $[\mathrm{mg} / \mathrm{dl}]{ }^{18,19}$.

Also, all dynamic PET-data were corrected for tumor motion during dynamic imaging, using the Image-Fusion-toolbox of the PMOD software package (PMOD Technologies Ltd., Zurich, Switzerland).

\section{PET analysis}

For each PET-CT scan, both static and dynamic, a tumor contour was generated using automated standardized-uptake-value (SUV) thresholding with the threshold depending on the tumor-to-background signal ratio with the gluteus muscle selected as a relevant background ${ }^{20,21}$. From the static PET-data, SUV $V_{\text {mean }}$ and SUV $V_{\text {max }}$ within the tumor were calculated using dedicated software (TrueD VC50, Siemens MI, Erlangen, Germany). For the dynamic PET-CT scans, a tumor contour was obtained from the last time frame of the dynamic PET-scan using PMOD (version 2.9, PMOD Technologies Ltd., Zurich, Switzerland). From the mean and maximum time activity curves (TACS) within the tumor, the FDG uptake rates ( $\triangle \mathrm{SUV} / \mathrm{min}$.) were calculated over the last 8 time frames of the dynamic PET-data. Subsequently, changes in the metabolic activity of the tumor, SUVs and FDG uptake rates, were quantified by calculation of the response indices (RIs), representing the percentage reduction relative to the pretreatment measured value.

\section{Statistical Analysis}

Statistical analyses were performed using SPSS (version 15.0; SPSS Inc., Chicago, IL, USA). All quantitative values were expressed as mean \pm standard deviation (SD) and range (min. to max.). Comparisons of related measurements were performed using a Wilcoxon-signed rank test, whereas a Mann-Whitney $U$ test was used in case of independent samples.

\subsection{Results}

\section{Metabolic treatment response during CRT}

A significant decrease of the metabolic activity within the tumor was observed already early during pre-operative CRT (Fig. 2.2A). SUV mean and SUV $V_{\text {max }}$ decreased from respectively $8.5 \pm 2.8$ (range: 4.0 to 15.1 ) and $16.4 \pm 5.8$ (range: 7.0 to 28.1 ) measured prior to the onset of CRT to $6.9 \pm 2.2$ (range: 4.3 to 12.7$)(p<0.001)$ and $13.0 \pm 4.8$ (range: 7.6 to $27.4)(p<0.001)$ after the first week of CRT. Comparable time-trends were found for the 
mean and maximum FDG uptake rates within the tumor, with RIs of respectively $21.0 \pm 31.3 \%$ (range: -40.6 to $65.2 \%)(p=0.003)$ and $11.4 \pm 41.5 \%$ (range: -73.1 to $60.7 \%$ ) $(p=0.062)$ after the first week of CRT (Fig. 2.2A).

\section{Metabolic treatment response during RT}

In contrast to CRT, for the patients pre-operatively treated with RT, no significant reduction of the tumors metabolic activity $\left(S U V_{\text {mean }} \& S U V_{\text {max }}\right)$ was observed early during therapy (Fig. 2.2B). Average values of respectively $8.3 \pm 2.9$ (range: 3.4 to 15.1 ) and $15.3 \pm 5.7$ (range: 5.7 to 29.3 ) were found for the pre-treatment PET-CT scan and $8.2 \pm 2.7$ (range: 4.4 to 14.3$)(p=0.767)$ and $14.7 \pm 5.2$ (range: 6.9 to 27.5$)(p=0.434)$ for the follow-up PET-data. For the mean and maximum FDG uptake rate of the tumor, RIs of respectively $-9.9 \pm 30.5 \%$ (range: -52.6 to $44.9 \%)(p=0.334)$ and $-6.7 \pm 22.4 \%$ (range: 41.2 to $33.0 \%)(p=0.293$ ) were found (Fig. 2.2B).

\section{A: Chemoradiotherapy (CRT)}

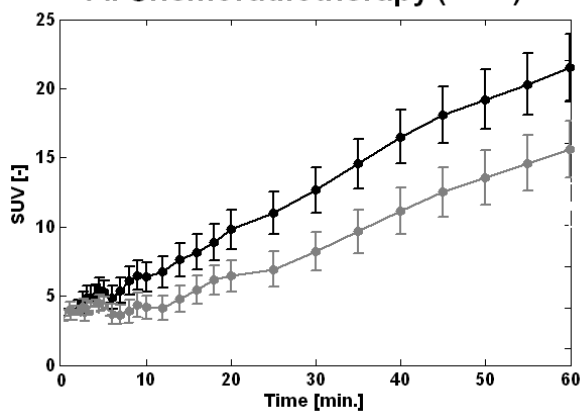

B: Radiotherapy (RT)

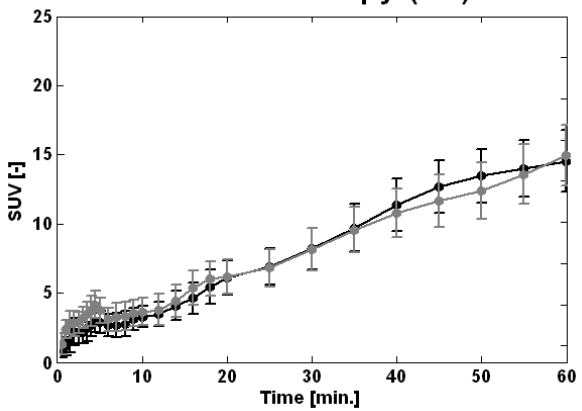

Figure 2.2: Mean time activity curves (TACS) of the tumor, indicating the amount and rate of FDG uptake over time. (A) Mean TACs at the two imaging time-points for the patients treated with chemo-radiotherapy, respectively pre-treatment (black) and one week (grey) after the onset of treatment, indicating a significant reduction of the metabolic activity of the tumor already during the week of treatment. (B) Mean TACs at both time-points for the patients treated with short course hypofractionated radiotherapy, respectively pretreatment (black) and after one week of treatment (grey), presenting stable FDG uptake levels within the tumor during radiotherapy treatment.

\section{CRT versus RT}

When comparing the metabolic treatment response of the two different treatment schemes after one week of treatment, a significant higher metabolic response was found for the patients treated with CRT when compared to pre-operative treatment with RT alone (Fig. 2.3). The average reduction of SUV $\mathrm{V}_{\text {mean }}$ of $16.4 \pm 18.2 \%$ (range: -20.9 to $47.6 \%$ ) after the first week of CRT was found to be statistically significantly higher when compared to the average reduction of only $0.1 \pm 14.7 \%$ (range: -29.4 to $23.5 \%$ ) for the patients treated with RT alone ( $\mathrm{P}=0.004)$ (Fig. 2.3). Also, the percent reduction of SUV $_{\max }$ of $17.6 \pm 20.5 \%$ (range: -21.8 to $50.3 \%$ ) after one week of CRT was found to be statistically significant higher than the average reduction of SUV $_{\max }$ of $1.8 \pm 15.4 \%$ (range: -21.1 to $27.1 \%$ ) after one week of RT alone ( $P=0.009)$ (Fig. 2.3). 


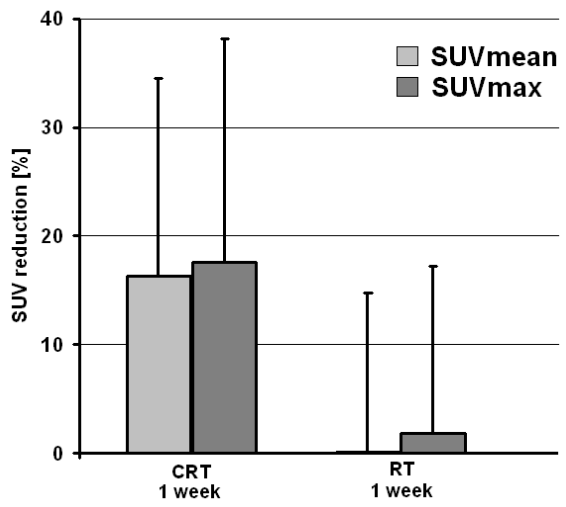

Figure 2.3: Average reductions of $S U V_{\text {mean }}$ and $S U V_{\max }$ within the tumor after one week of treatment for the patients treated with respectively chemo-radiotherapy (CRT) and short course hypofractionated radiotherapy (RT).

\subsection{Discussion}

In the present report we provide first evidence that the significant reduction of the metabolic activity of the tumor, as seen early during pre-operative CRT might be induced by the chemotherapeutic agent Capecitabine, since no such changes in the metabolic activity of the tumor could be determined after one week of treatment with RT alone, despite the much higher applied BED. When looking at only the dose levels of RT, a higher metabolic treatment response was expected for the patients treated with hypofractionated RT at the time of the follow-up PET-CT scan as these patients received a higher $B E D$ when compared to the patients treated with CRT. However, this study presents with opposite findings. These findings imply that the addition of the chemotherapeutic agent Capecitabine to RT-treatment can induce the early metabolic decrease in the FDG-uptake, which is more and more used as a predictor of the pathological treatment response. A comparative study, investigating the metabolic activity of the tumor early during chemotherapy alone was unfortunately not feasible, because initial chemotherapy alone is not standard care in our region. However, earlier clinical studies have already indicated important and even prognostic significant differences in FDG uptake as early as one to three weeks after the first cycle of chemotherapy in various cancer types ${ }^{22-26}$. For chemotherapy with 5-FU, a comparable chemotherapeutic drug to Capecitabine, a consistent decrease in FDG uptake by $50 \%$ was already present as early as 3 days after the start of the chemotherapy ${ }^{27}$. In contrast, chemotherapeutic agents like doxorubicin or paclitaxel increased FDG uptake ${ }^{27}$. The early reduction in FDG uptake under 5-FU treatment might be related to a decreased activity of either the glucose transporter Glut-1 or the phosphorylation enzyme hexokinase ${ }^{27}$. Also for rectal cancer patients, a significant reduction of FDG uptake was observed when applying chemotherapy alone without additional RT ${ }^{28-30}$. Sharma et al. presented data demonstrating a significant reduction of the hexokinase activity in colorectal tumor cells after treatment with chemotherapy ${ }^{31}$. Hexokinase is an enzyme known 
to phosphorylate six-carbon sugars, including FDG, making it unable to move or be transported out of the cell ${ }^{31}$. A reduction of the hexokinase concentration leads to a decreased amount of FDG trapped within the cells resulting in decreased SUVs ${ }^{31}$. The degree of chemotherapy-induced changes in metabolic activity of colorectal tumors was shown to be highly predictive for patient outcome ${ }^{30}$. Such FDG uptake measurements provide a valuable surrogate for the intratumoral biodistribution of the drug within solid tumors and thereby also for the intratumoral effectiveness. A homogeneous intratumoral biodistribution of the drug Capecitabine is an important prerequisite for its effectiveness as a radiosensitizing cancer cell agent during $\mathrm{RT}^{32}$.

In contrast to chemotherapeutic agents, RT alone on cancer cells does not lead to early changes in its glucose transport or cellular hexokinase activity ${ }^{32}$. Instead, RT induces changes on the cellular cell cycle, the DNA repair and apoptosis, all of which do probably not lead to early changes in the FDG uptake of cancer cells, as seen in our study $^{32}$. Thus, the metabolic changes in PET images after the first week of CRT in rectal cancer might be more seen as activity changes in the cells ability to incorporate glucose under the influence of the chemotherapeutic drug rather than as RT-induced cytotoxicity.

This is the first study presenting significant different reaction patterns of rectal tumors to pre-operative treatment with either combined CRT or RT alone already early during treatment. Knowledge about early reaction patterns within malignancies during pre-operative treatment is important for further evaluation of PET-based response predictions which are the first elements of individualized treatment schemes in the near future.

One published study presented with contradicting results as they found a significant reduction in the FDG uptake after a short course hypofractionated RT ${ }^{9}$. One of the differences between the study of Siegel et al. and our study was the later timepoint of 2 days of the follow-up PET-CT scan ${ }^{9}$. However, the average difference of only two days is very unlikely to explain such a dramatic reduction of more than $35 \%$ for the SUV $_{\max }$. Instead, another explanation for the discrepancy of the results might be the reproducibility of SUV determination ${ }^{12,18,20,33-36}$. The patients BGL at the time of PETimaging and the time interval between FDG injection and the start of PET-imaging are known to influence the SUVs resulting from PET-analysis ${ }^{12,18,33-36}$. The study of Siegel et al. did not perform a normalization of the sequential PET-data for the patient's BGL at the time of the PET-imaging ${ }^{9}$. A lack of BGL-normalization however could in cases of large intra-patient BGL fluctuations lead to misinterpretations of the SUV time-trends 12, 33-36. Also, Siegel et al. performed only static PET-acquisition approximately 60 minutes after intravenous injection of FDG. The major drawback of static PET-imaging however is the time-dependency of SUV determination because of continuous FDG uptake within the tumor for several hours after FDG injection ${ }^{12,33}$. The use of an identical time-interval between FDG injection and the start of PET-imaging is essential when performing sequential FDG-PET-CT-scans for metabolic response evaluations ${ }^{12}$. To overcome this time dependency, we additionally performed dynamic PET-imaging in the majority of the study patients. By looking at the average FDG uptake rate within the tumor, before reaching a plateau, instead of only at a SUV at a single time point, the time dependency of SUV determination no longer influences the time-trends of FDG uptake during therapy. Another important confounder in the use of PET-imaging is 
a peritumoral inflammatory reaction, as inflammatory cells are known to avidly consume FDG ${ }^{11,37}$. An increased FDG uptake by inflammatory cells in the direct neighborhood of the tumor can lead to an underestimation of the SUV decrease within the tumor $^{8,11}$. To ensure a reliable comparison of the metabolic treatment response of the two treatment schemes without bias due to inflammatory reactions, only patients without a visually observable peritumoral inflammatory reaction were included in this study. As described, the patients included in this study were treated according to the national guidelines with either short course hypofractionated RT or CRT, based on their clinical TNM stage and predicted CRM determined from MR-imaging. So, the included patients were not enrolled in a randomized two-arm trial. However, this was not expected to result in a bias of the results of this study. The patients diagnosed with locally advanced rectal cancer presented with higher tumor volumes compared to the patients diagnosed with non-locally advanced rectal cancer. Larger tumors are more likely to have hypoxic regions, being less sensitive to radiotherapy treatment. So, a possible bias would have more likely resulted in a higher metabolic treatment response in the smaller non-locally advanced rectal tumors treated with a high BED when compared to the larger locally advanced rectal tumors treated with a relatively low BED, which is the opposite of our findings.

In conclusion, already after the first week of chemo-radiotherapy, the metabolic activity of the tumor was found to decrease significantly, which was not detectable early after treatment with radiotherapy alone, suggesting the chemotherapeutic agent Capecitabine as the primary initiator of the observed reduction of the tumors metabolic activity. 


\subsection{References}

[1] Kalff, V, Duong, C, Drummond, EG, Matthews, JP, Hicks, RJ. Findings on 18F-FDG PET scans after neoadjuvant chemoradiation provides prognostic stratification in patients with locally advanced rectal carcinoma subsequently treated by radical surgery. J Nucl Med 2006;47:14-22.

[2] Cascini, GL, Avallone, A, Delrio, P, et al. 18F-FDG PET is an early predictor of pathologic tumor response to preoperative radiochemotherapy in locally advanced rectal cancer. J Nucl Med 2006;47:1241-1248.

[3] Capirci, C, Rampin, L, Erba, PA, et al. Sequential FDG-PET/CT reliably predicts response of locally advanced rectal cancer to neo-adjuvant chemo-radiation therapy. Eur J Nucl Med Mol Imaging 2007;34:1583-1593.

[4] Capirci, C, Rubello, D, Chierichetti, F, et al. Long-term prognostic value of 18F-FDG PET in patients with locally advanced rectal cancer previously treated with neoadjuvant radiochemotherapy. AJR Am J Roentgenol 2006;187:W202-208.

[5] Amthauer, H, Denecke, T, Rau, B, et al. Response prediction by FDG-PET after neoadjuvant radiochemotherapy and combined regional hyperthermia of rectal cancer: correlation with endorectal ultrasound and histopathology. Eur J Nucl Med Mol Imaging 2004;31:811-819.

[6] Denecke, T, Rau, B, Hoffmann, KT, et al. Comparison of CT, MRI and FDG-PET in response prediction of patients with locally advanced rectal cancer after multimodal preoperative therapy: is there a benefit in using functional imaging? Eur Radiol 2005;15:1658-1666.

[7] Guillem, JG, Moore, HG, Akhurst, T, et al. Sequential preoperative fluorodeoxyglucose-positron emission tomography assessment of response to preoperative chemoradiation: a means for determining longterm outcomes of rectal cancer. J Am Coll Surg 2004;199:1-7.

[8] Rosenberg, R, Herrmann, K, Gertler, R, et al. The predictive value of metabolic response to preoperative radiochemotherapy in locally advanced rectal cancer measured by PET/CT. Int J Colorectal Dis 2009;24:191-200.

[9] Siegel, R, Dresel, S, Koswig, S, et al. Response to preoperative short course radiotherapy in locally advanced rectal cancer: value of f-fluorodeoxyglucose positron emission tomography. Onkologie 2008;31:166-172.

[10] Vliegen, RF, Beets-Tan, RG, Vanhauten, B, et al. Can an FDG-PET/CT Predict Tumor Clearance of the Mesorectal Fascia after Preoperative Chemoradiation of Locally Advanced Rectal Cancer? Strahlenther Onkol 2008;184:457-464.

[11] Janssen, $\mathrm{MH}$, Ollers, MC, Riedl, RG, et al. Accurate prediction of pathological rectal tumor response after 2 weeks of pre-operative radiochemotherapy using FDG-PET-CT imaging. Int J Radiat Oncol Biol Phys 2010; 77: 392-399.

[12] Hindie, E, Hennequin, C, Moretti, JL. Predicting response to chemoradiotherapy in rectal and oesophageal cancer with 18F-FDG: prognostic value and possible role in patient management. Eur J Nucl Med Mol Imaging 2007;34:1576-1582.

[13] Valentini, V, Coco, C, Cellini, N, et al. Ten years of preoperative chemoradiation for extraperitoneal T3 rectal cancer: acute toxicity, tumor response, and sphincter preservation in three consecutive studies. Int J Radiat Oncol Biol Phys 2001;51:371-383.

[14] Roedl, JB, Colen, RR, Holalkere, NS, Fischman, AJ, Choi, NC, Blake, MA. Adenocarcinomas of the esophagus: response to chemoradiotherapy is associated with decrease of metabolic tumor volume as measured on PET-CT. Comparison to histopathologic and clinical response evaluation. Radiother Oncol 2008;89:278-286.

[15] Petit, SF, Aerts, HJ, van Loon, JG, et al. Metabolic control probability in tumour subvolumes or how to guide tumour dose redistribution in non-small cell lung cancer (NSCLC): an exploratory clinical study. Radiother Oncol 2009;91:393-398.

[16] Glimelius, B, Gronberg, H, Jarhult, J, Wallgren, A, Cavallin-Stahl, E. A systematic overview of radiation therapy effects in rectal cancer. Acta Oncol 2003;42:476-492.

[17] Janssen, MH, Aerts, HJ, Ollers, MC, et al. Tumor Delineation Based on Time-Activity Curve Differences Assessed with Dynamic Fluorodeoxyglucose Positron Emission TomographyComputed Tomography in Rectal Cancer Patients. Int J Radiat Oncol Biol Phys 2009; 73: 456-465. 
[18] Beaulieu, S, Kinahan, P, Tseng, J, et al. SUV varies with time after injection in (18)F-FDG PET of breast cancer: characterization and method to adjust for time differences. J Nucl Med 2003;44:1044-1050.

[19] Eary, JF, Mankoff, DA. Tumor metabolic rates in sarcoma using FDG PET. J Nucl Med 1998;39:250254.

[20] Ollers, M, Bosmans, G, van Baardwijk, A, et al. The integration of PET-CT scans from different hospitals into radiotherapy treatment planning. Radiother Oncol 2008;87:142-146.

[21] Daisne, JF, Sibomana, M, Bol, A, Doumont, T, Lonneux, M, Gregoire, V. Tri-dimensional automatic segmentation of PET volumes based on measured source-to-background ratios: influence of reconstruction algorithms. Radiother Oncol 2003;69:247-250.

[22] Kostakoglu, L, Coleman, M, Leonard, JP, Kuji, I, Zoe, H, Goldsmith, SJ. PET predicts prognosis after 1 cycle of chemotherapy in aggressive lymphoma and Hodgkin's disease. J Nucl Med 2002;43:1018-1027.

[23] Juweid, ME, Cheson, BD. Positron-emission tomography and assessment of cancer therapy. N Engl J Med 2006;354:496-507.

[24] Ott, K, Fink, U, Becker, K, et al. Prediction of response to preoperative chemotherapy in gastric carcinoma by metabolic imaging: results of a prospective trial. J Clin Oncol 2003;21:4604-4610.

[25] Weber, WA, Ott, K, Becker, K, et al. Prediction of response to preoperative chemotherapy in adenocarcinomas of the esophagogastric junction by metabolic imaging. J Clin Oncol 2001;19:3058-3065.

[26] Weber, WA, Petersen, V, Schmidt, B, et al. Positron emission tomography in non-small-cell lung cancer: prediction of response to chemotherapy by quantitative assessment of glucose use. J Clin Oncol 2003;21:2651-2657.

[27] Direcks, WG, Berndsen, SC, Proost, N, et al. [18F]FDG and [18F]FLT uptake in human breast cancer cells in relation to the effects of chemotherapy: an in vitro study. Br J Cancer 2008;99:481-487.

[28] Dimitrakopoulou-Strauss, A, Strauss, LG, Burger, C, et al. Prognostic aspects of 18F-FDG PET kinetics in patients with metastatic colorectal carcinoma receiving FOLFOX chemotherapy. J Nucl Med 2004;45:1480-1487.

[29] Dimitrakopoulou-Strauss, A, Strauss, LG, Rudi, J. PET-FDG as predictor of therapy response in patients with colorectal carcinoma. Q J Nucl Med 2003;47:8-13.

[30] de Geus-Oei, LF, van Laarhoven, HW, Visser, EP, et al. Chemotherapy response evaluation with FDG-PET in patients with colorectal cancer. Ann Oncol 2008;19:348-352.

[31] Sharma, RI, Smith, TA. Colorectal tumor cells treated with 5-FU, oxaliplatin, irinotecan, and cetuximab exhibit changes in 18F-FDG incorporation corresponding to hexokinase activity and glucose transport. J Nucl Med 2008;49:1386-1394.

[32] Schoder, H, Ong, SC. Fundamentals of molecular imaging: rationale and applications with relevance for radiation oncology. Semin Nucl Med 2008;38:119-128.

[33] Keyes, JW, Jr. SUV: standard uptake or silly useless value? J Nucl Med 1995;36:1836-1839.

[34] Langen, KJ, Braun, U, Rota Kops, E, et al. The influence of plasma glucose levels on fluorine-18fluorodeoxyglucose uptake in bronchial carcinomas. J Nucl Med 1993;34:355-359.

[35] Lindholm, P, Minn, H, Leskinen-Kallio, S, Bergman, J, Ruotsalainen, U, Joensuu, H. Influence of the blood glucose concentration on FDG uptake in cancer--a PET study. J Nucl Med 1993;34:1-6.

[36] Crippa, F, Gavazzi, C, Bozzetti, F, et al. The influence of blood glucose levels on [18F]fluorodeoxyglucose (FDG) uptake in cancer: a PET study in liver metastases from colorectal carcinomas. Tumori 1997;83:748-752.

[37] Kao, PF, Chou, YH, Lai, CW. Diffuse FDG uptake in acute prostatitis. Clin Nucl Med 2008;33:308310. 



\title{
Chapter
}

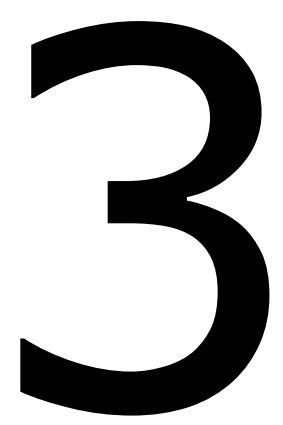

\section{Accurate prediction of pathological rectal tumor response after 2 weeks of pre-operative chemo- radiotherapy using FDG-PET-CT imaging.}

\author{
Marco Janssen \\ Michel Öllers \\ Robert Riedl \\ Jørgen van den Bogaard \\ Jeroen Buijsen \\ Ruud van Stiphout \\ Hugo Aerts \\ Philippe Lambin \\ Guido Lammering
}

International Journal of Radiation Oncolology * Biology * Physics, 2010; 77(2): 392-399 


\section{Abstract \\ Purpose}

To study the optimal time point for repeated FDG-PET-CT imaging during pre-operative chemo-radiotherapy (CRT) as well as the best predictive factor for the prediction of pathological treatment response in locally advanced rectal cancer.

\section{Materials \& Methods}

In total, thirty patients, referred for pre-operative CRT treatment, were included in this prospective study. All patients underwent sequential FDG-PET-CT imaging at four time points: prior to therapy, at day 8 and 15 during CRT and shortly before surgery. The tumors metabolic treatment response was correlated with the pathological response by evaluation of the tumor regression grade (TRG) and the ypT-stage of the resected specimen.

\section{Results}

Based on the TRG, thirteen patients were classified as pathological responders (TRG 12), whereas seventeen patients were classified as pathological non-responders (TRG 3$5)$. The response-index (RI) for the maximum standardized uptake value $\left(S_{U} V_{\max }\right)$ on day 15 of CRT was found to be the best predictive factor for the pathological response ( $A U C=0.87$ ) compared to the RI on day 8 ( $A U C=0.78$ ) or the RI of pre-surgical PETimaging ( $A \cup C=0.66$ ). A cut-off value of $43 \%$ for the reduction of $S U V_{\max }$ resulted in a sensitivity of $77 \%$ and a specificity of $93 \%$.

\section{Conclusions}

The SUV $\mathrm{Vax}_{\text {max }}$ based RI calculated after the first two weeks of CRT provided the best predictor of pathological treatment response reaching AUC's of 0.87 and 0.84 for the TRG and the ypT-stage, respectively. However, a few patients presented with peritumoral inflammatory reactions, which led to mispredictions. Excluding these patients further enhanced the predictive accuracy of PET-imaging to AUCs of 0.97 and 0.89 for TRG and ypT, respectively. 


\subsection{Introduction}

In patients diagnosed with locally-advanced-rectal-cancer (LARC), pre-operative chemo-radiotherapy (CRT) has become an established standard procedure ${ }^{1-3}$. Importantly, however, during the last years, pre-operative CRT has been shown to not only reduce the risk for local recurrence, but to also induce a significant tumor downsizing and downstaging ${ }^{4-6}$. In $15-30 \%$ of the patients, even a pathological complete tumor regression has been observed ${ }^{4-7}$.

Interestingly, first correlations between the reduction of the fluorodeoxyglucose (FDG) uptake within the tumor and the pathological tumor response after CRT have been reported by some groups ${ }^{5-14}$. Most of these studies performed positronemission-tomography computed tomography (PET-CT) scans before the start and after the finish of the pre-operative $\mathrm{CRT}$, correlating semi-quantitative measurements of FDG uptake with the tumor-regression-grade (TRG) ${ }^{5-12}$.

For the clinical practice, however, an earlier prediction of the pathological tumor response would be even more attractive, because it could enable response-guided modifications of the treatment protocol, based on FDG uptake changes, possibly strengthened by clinical or biological factors ${ }^{13-15}$. Until now, only two studies have reported on an early prediction of the pathological tumor response based on PET-CT imaging during pre-operative CRT ${ }^{13-14}$. Cascini et al. showed that early changes in the metabolic activity of the tumor, measured 12 days after the start of pre-operative treatment with CRT, were predictive for the pathological treatment response in rectal cancer ${ }^{13}$. Rosenberg et al. presented a correlation of both the early metabolic response evaluation as well as the late metabolic response evaluation with the histopathological tumor response, with the accuracy of the late metabolic response being marginally superior ${ }^{14}$. Both studies used a protocol, in which the second PET-CT scan was performed at the end of the second week during CRT ${ }^{13-14}$.

However, no studies have yet examined other time points of PET-imaging during pre-operative CRT in order to define the best prediction of pathological tumor response as advised by Hindie et al. ${ }^{15}$. Thus, we initiated this study, in which we performed PET-CT scans at two different time points during pre-operative CRT as well as a pre-surgical PET-CT scan, in order to investigate the optimal time point of PET-imaging during pre-operative CRT as well as to define the best PET-criteria resulting in the best prediction of pathological tumor response.

\subsection{Materials \& Methods}

\section{Patient Characteristics}

Thirty patients diagnosed with non-metastasized LARC were included in this study, from which the clinical TN staging was evaluated on a pre-treatment magnetic resonance (MR) scan (Table 3.1). All patients were pre-operatively treated with radiotherapy (28 fractions of $1.8 \mathrm{~Gy}, 5$ fractions/week) and concomitant chemotherapy (Capecitabine $825 \mathrm{mg} / \mathrm{m}^{2} \mathrm{BID}$ ), followed by a total mesorectal excision. As a part of the study, all patients also underwent sequential FDG-PET-CT imaging at four different time points: prior to therapy, on day 8 and day 15 of CRT and once shortly before surgery. 
Due to technical problems or patient incompliance, not all PET-CTs could be performed as planned. Three patients refused PET-CT imaging on day 15 and for one patient no FDG could be injected on the second PET-CT scan. For seven patients, no PET-CT scan could be performed prior to surgery. According to the Dutch law, the Medical Ethics Committee approved the trial. All patients gave written informed consent before entering the study.

\section{PET-CT Imaging and Processing}

All PET-CT scans were performed by use of a dedicated Siemens Biograph 40 TruePoint PET-CT simulator (Siemens Medical, Erlangen, Germany) with an axial field of view of $16.2 \mathrm{~cm}$, slice thickness of $3 \mathrm{~mm}$ and a pixel spacing of $5.3456 \mathrm{~mm}$ in both directions. The scanner is equipped with ultra-fast detector electronics (Pico3D) and has a spatial resolution of approximately $6 \mathrm{~mm}$ full-width-at-half-maximum (FWHM). PET-imaging was done in 3D, requiring a proper scatter correction. CT-based attenuation and decay correction was performed. PET images were reconstructed from the acquired listmode (LM) data using Fourier-rebinning (FORE) and ordered-subset-expectationmaximization-reconstruction (OSEM 2D) with 4 iterations and 8 subsets. After a fasting period of at least 6 hours prior to FDG injection, FDG was injected intravenously, with the activity normalized for the weight of the patient (weight $[\mathrm{kg}]^{*} 4+20[\mathrm{MBq}]$ ), followed by injection of physiologic-saline $(10 \mathrm{ml})$. After an uptake period of 60 minutes, the patient was positioned on a flat tabletop using a movable laser alignment system in a "head-first supine" position with the arms crossed above the chest. A PET-CT-scan of the abdominal region was performed using an acquisition time of 5 minutes per bed position. Additionally, all PET-data were normalized for the blood glucose level measured shortly before FDG administration ${ }^{16}$.

\section{PET Analysis}

For each of the PET-scans, a tumor contour was generated using automated standardized-uptake-value (SUV) thresholding with the threshold (percentage of SUV $\mathrm{Vax}_{\text {mithin }}$ the tumor) depending on the tumor-to-background signal ratio with the gluteus muscle selected as relevant background ${ }^{17,18}$. Dedicated software (TrueD, Siemens Medical, Erlangen, Germany) was used to calculate the SUV $V_{\text {mean }}$ and SUV $V_{\max }$ within the tumor. Subsequently, the response indices (RIs), indicating the percentage reduction relative to the pre-treatment measured value, were calculated and correlated to the pathological tumor response. If no residual metabolic activity was present on the presurgical PET-CT scan, the patient was classified as a metabolic complete responder (mCR) (Fig. 3.1A) and the SUV was defined as being zero and the RI was set as $100 \%$ for the pre-surgical PET-scan.

\section{Pathological Tumor Response}

For each tumor, the pathological tumor response was evaluated by determining the tumor-regression-grade (TRG) as proposed by Mandard ${ }^{19}$. All tumors were retrospectively classified by an experienced pathologist (RR), who was blinded for the PET-data, as follows: TRG1, complete tumor response; TRG2, residual cancer cells scattered through fibrosis; TRG3, an increased number of residual cancer cells, with predominant fibrosis; TRG4, residual cancer outgrowing fibrosis; TRG5, no regressive changes within 
the tumor. Based on the TRGs, the tumors were grouped into responders (TRG1, 2) and non-responders (TRG 3-5). Furthermore, the pathological TN -classification (ypTN) was collected from the pathological reports. Subsequently, the patients were also subdivided into a group with ypT0, yрT1 and ypT2 stages and a group with a ypT3 or a ypT4 stage.

\section{Statistical Analysis}

Statistical analyses were performed using SPSS (version 15.0; SPSS Inc., Chicago, IL, USA). All quantitative values were expressed as mean \pm standard deviation (SD) and range ( $\mathrm{min}$. to max.). Comparisons of related measurements were performed using a Wilcoxon-signed rank test, whereas a Mann-Whitney $U$ test was used in case of independent samples. Receiver operating characteristics (ROC) analysis was performed to evaluate the optimal cut-off value used for the prediction of the pathologic treatment response.

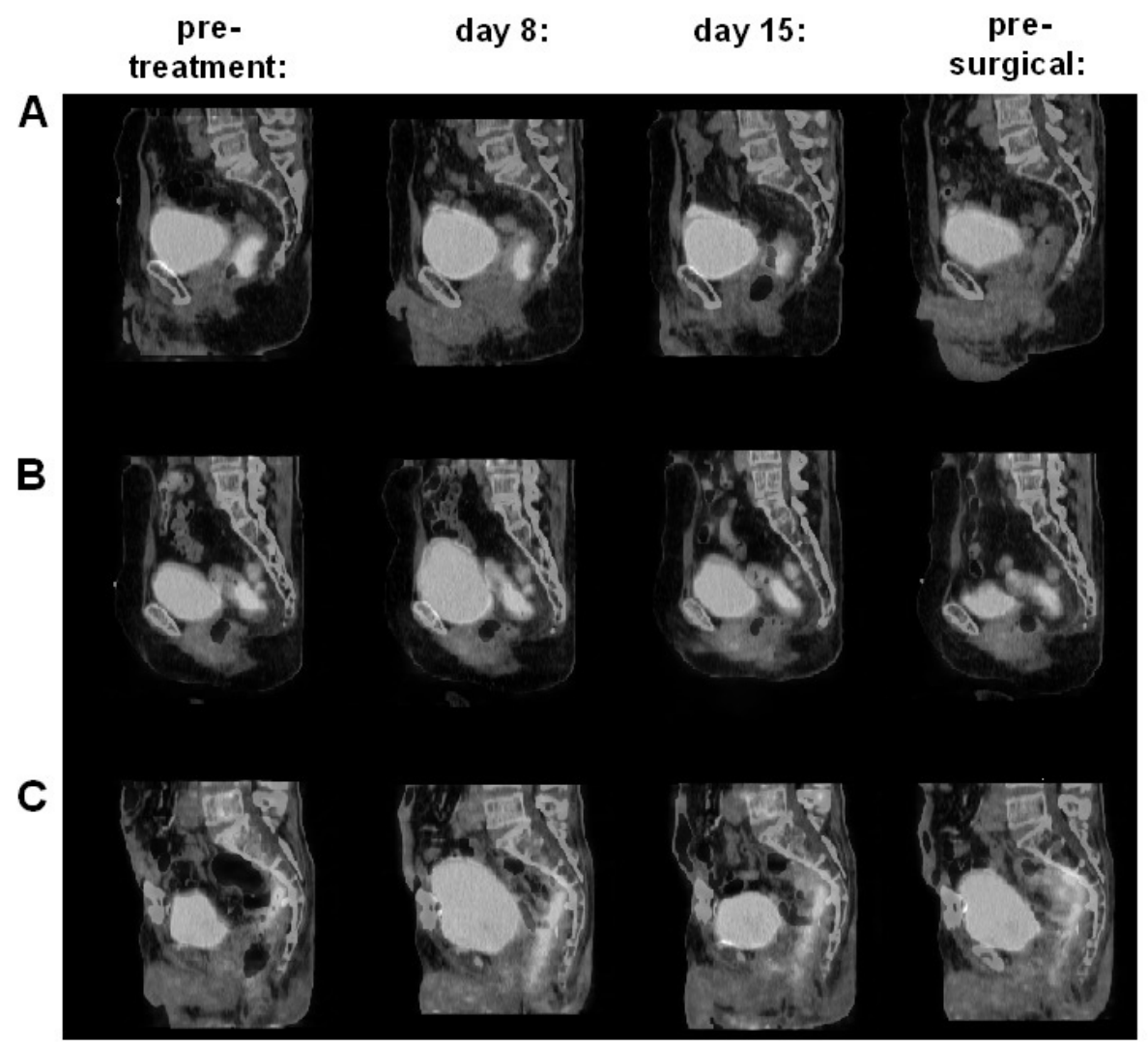

Figure 3.1: Representative FDG-PET-CT images at all four time points for a metabolic complete responder (A) a metabolic partial responder (B) and a patient with a pathological reported peritumoral inflammatory reaction (C). 


\subsection{Results}

\section{Metabolic Response Evaluation}

In general, the highest FDG uptake value was detected on the pre-treatment PET-CT scan, followed by a statistically significant reduction of the FDG uptake during preoperative CRT $(P<0.001)$ (Fig. 3.2A). Six of the included patients $(20 \%)$ were classified as $\mathrm{mCR}$ (Fig. 3.1A), whereas four patients (13\%) presented with an increased FDG uptake during CRT in the peritumoral tissues, indicating an inflammatory reaction (Fig. 3.1C). For these four patients, the histopathological reports also described a inflammatory reaction in the resected tumor specimen. Overall, an average $S_{\text {SU }}$ mean $8.3 \pm 2.8$ (range: 4.3 to 15.5 ) was calculated on the pre-treatment PET-scan. During preoperative CRT, the SUV mean within the tumor decreased to average values of $7.1 \pm 2.3$ (range: 4.2 to 12.7$)$ on day $8(P=0.002)$, to $5.7 \pm 1.7$ (range: 3.5 to 9.9$)$ on day 15 $(P<0.001)$ to $2.6 \pm 1.8$ (range: 0.0 to 5.1 ) on the pre-surgical scan $(P=0.001)$ (Fig. 3.2A). $\mathrm{SUV}_{\text {max }}$ showed a time trend comparable to SUV $\mathrm{V}_{\text {mean, }}$ with average values starting at 16.3 \pm 5.9 (range: 7.4 to 28.1 ) on day 0 , decreasing to $13.4 \pm 4.7$ (range: 8.1 to 27.4 ) on day 8 ( $P<0.001), 10.4 \pm 3.5$ (range: 6.0 to 18.9$)$ on day $15(P<0.001)$ to $5.4 \pm 3.8$ (range: 0.0 to 12.1 ) on the pre-surgical PET-scan $(P<0.001)$ (Fig. 3.2A).

\section{Pathologic response evaluation}

Thirteen of the included patients (43\%) were classified as pathological responders (4 TRG 1, 9 TRG 2), and seventeen patients (57\%) were classified as pathological nonresponders (11 TRG 3, 6 TRG 4) (Table 3.1). Based on the pathological reports, three specimens were classified as ypT0, one as ypT1, six as ypT2, twelve as ypT3 and two as ypT4 (Table 3.1).

\section{Correlation between the metabolic and pathological treatment response}

The following calculated RIs were found to be significantly different with respect to the

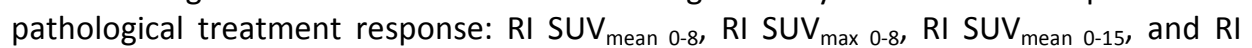
SUV $_{\max 0-15}\left(\right.$ Table 3.2). ROC-curve analysis for the response indices of both SUV $V_{\text {mean }}$ and SUV $_{\text {max }}$ at day 8 of CRT, revealed no cut-off value for the differentiation between pathological responders and non-responders due to a substantial overlap of the response indices relative to the TRG. However, the $\mathrm{RI}^{\prime} \mathrm{S}$ of $\mathrm{SUV}_{\max }$ based on the PETscan performed on day 15 of CRT were found to accurately differentiate between histo-pathological responders and non-responders, because less overlap was observed between the response indices relative to the TRG (Fig. 3.3). Using ROC-curve analysis, a cut-off value of $43 \% \mathrm{SUV}_{\max }$ reduction on day 15 resulted in a sensitivity of $77 \%$, a specificity of $93 \%$, a negative predictive value (NPV) of $82 \%$ and a positive predictive value (PPV) of $91 \%$. 
A

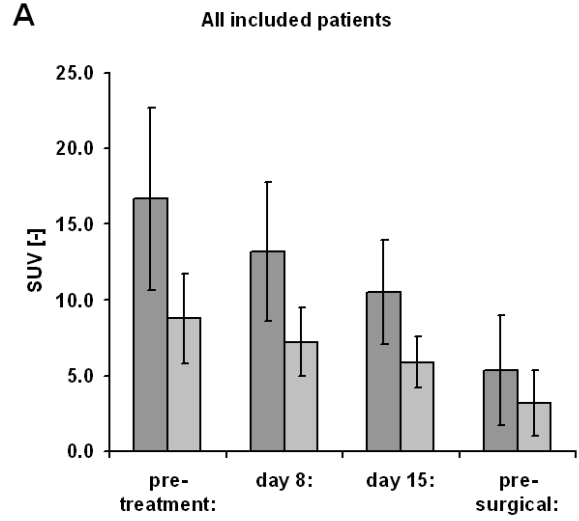

C

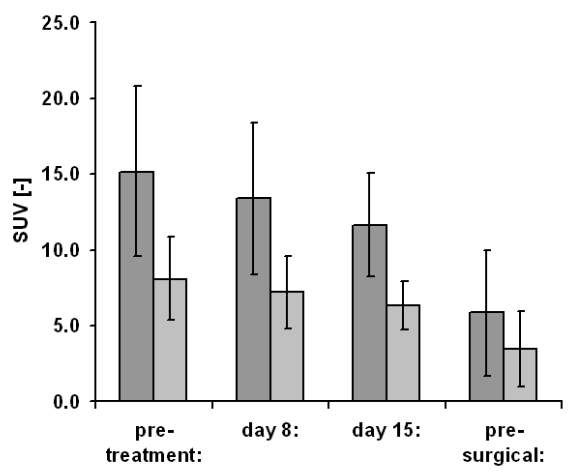

B Pathological Responders (TRG 1,2)

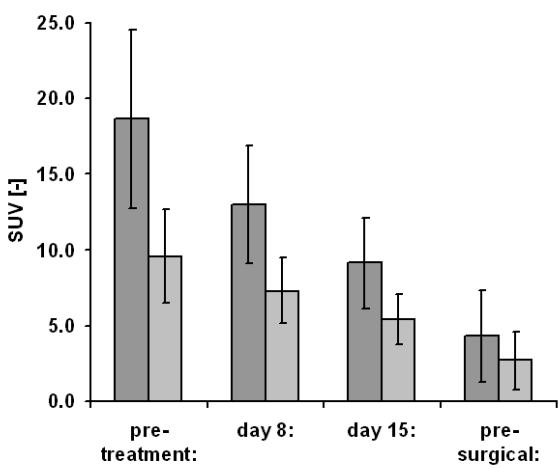

Figure 3.2: Overview of the $S U V_{\max }$ (dark boxes) and $S U V_{\text {mean }}$ (light boxes) time trends during pre-operative chemo-radiotherapy. (A) average time trends for all patients included in this study (B) average time trend for the pathological responding patients (TRG 1-2), indicating a strong reduction of the FDG uptake during the first two weeks of pre-operative treatment (C) average time trends of the pathological non-responding patients (TRG 3-5), showing less reduction of the FDG uptake during pre-operative treatment with chemoradiotherapy when compared to the pathological responding patients.

Correlating the PET-criteria and response indices with the ypT- stage revealed significant different values for three of the response indices: RI SUV $\max 0-8, R_{\text {I SUV }}$ mean 0-15 and

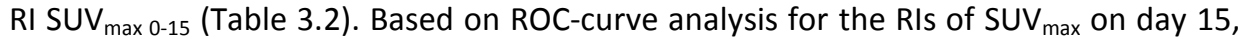
an optimal cut-off value of $43 \%$ was defined, resulting in a sensitivity of $57 \%$, a specificity of $85 \%$, a NPV of $65 \%$ and a PPV of $80 \%$ (Fig. 3.4). By excluding the patients with a perceived peritumoral inflammatory response from further analysis, the used models for the prediction of both the TRG and the ypT-classification improved from an area under the curve (AUC) of respectively $0.87 \pm 0.07$ and $0.84 \pm 0.08$ to AUCs of $0.97 \pm 0.04$ and $0.89 \pm 0.08$ (Fig. 3.3A \& 3.4A). 
Table 3.1: Overview of the clinical (c) and pathological (yp) tumor staging (TN(M)), the tumor regression grade (TRG) and the response index (RI) of SUV $\mathrm{max}_{\max }$ during the first 15 days of pre-operative treatment with chemo-radiotherapy.

\begin{tabular}{|c|c|c|c|c|}
\hline Patient & cTNM & ypTNM & TRG & RI SUV $\max 0-15$ \\
\hline 1 & T3N1M0 & T3NO & 3 & \\
\hline 2 & T2N1M0 & TONO & 1 & 51.9 \\
\hline 3 & T3N2M0 & T3N1 & 3 & 41.7 \\
\hline 4 & T3N2M0 & T2NO & 2 & 69.4 \\
\hline 5 & T3N1M0 & T3NO & 3 & \\
\hline 6 & T4N2M0 & T3NO & 2 & 38.9 \\
\hline 7 & T3N1M0 & T2NO & 2 & 64.8 \\
\hline 8 & T3N2M0 & T2NO & 2 & -2.4 \\
\hline 9 & T3N2M0 & T2NO & 3 & 31.5 \\
\hline 10 & T3N2MO & T4NO & 3 & -11.8 \\
\hline 11 & T3N2M0 & T3NO & 3 & 47.6 \\
\hline 12 & T3N2M0 & T3N1 & 4 & 14.4 \\
\hline 13 & T3N1M0 & TONO & 1 & 70.4 \\
\hline 14 & T3N2M0 & T3NO & 4 & 28.8 \\
\hline 15 & T3N1M0 & T2NO & 3 & 39.7 \\
\hline 16 & T3NOMO & T2NO & 3 & 5.1 \\
\hline 17 & T3N2M0 & T3N1 & 3 & 35.9 \\
\hline 18 & T3N1M0 & T1N0 & 2 & 9.7 \\
\hline 19 & T3N2M0 & T3N2 & 3 & 33.4 \\
\hline 20 & T3N2M0 & T3N2 & 4 & 28.6 \\
\hline 21 & T3N2MO & TONO & 1 & 54.6 \\
\hline 22 & T3N2M0 & T3N1 & 2 & 45.5 \\
\hline 23 & T3NOMO & T3N1 & 4 & 5.2 \\
\hline 24 & T3N1M0 & T3N2 & 3 & -8.2 \\
\hline 25 & T4N1M0 & T4NO & 4 & -15.7 \\
\hline 26 & T3N1M0 & T2NO & 2 & 48.6 \\
\hline 27 & T3NOMO & TONO & 1 & 68.4 \\
\hline 28 & T3N2M0 & $\mathrm{T} 2 \mathrm{~N} 2$ & 2 & 45.6 \\
\hline 29 & T3N2M0 & T2NO & 2 & 46.7 \\
\hline 30 & T3N1M0 & T3N1 & 4 & -7.1 \\
\hline
\end{tabular}


The prediction of TRG improved to a sensitivity of $90 \%$, a specificity of $93 \%$, a NPV of 93\% and a PPV of $90 \%$, whereas the prediction of the ypT-classification improved to a sensitivity of $80 \%$, a specificity of $85 \%$, a NPV of $80 \%$ and PPV of $85 \%$. The tumors with a $\mathrm{mCR}$ after CRT did not all correlate with a pathological complete response (pCR). Only one out of six patients demonstrating a $\mathrm{MCR}$ on the pre-surgical performed PETCT scan completely responded on pathology (TRG 1). The other five tumors turned out to be pathological partial responding malignancies (1 TRG 2 and 4 TRG 3).

A

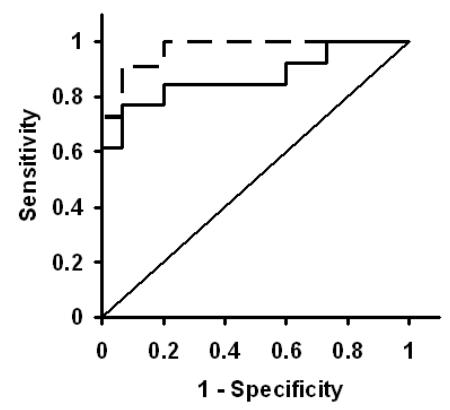

B

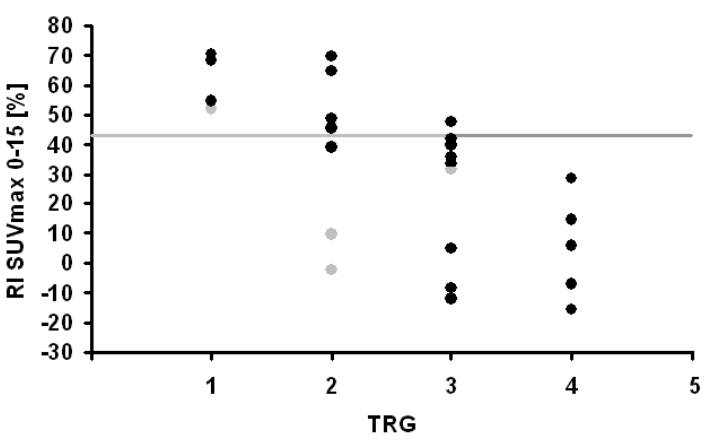

Figure 3.3: A) ROC-curves of the SUV $_{\max }$ response-index on day 15 of the chemo-radiotherapy relative to the TRG before (solid, AUC $=0.87$ ) and after (dashed, AUC $=0.97$ ) exclusion of the patients with a reported peritumoral inflammatory response. B) Response indices of $S U V_{\max }$ on day 15 relative to the TRG-stage. The grey horizontal line indicates the ROC-curve analysis based the cut-off value of $43 \%$ differentiating pathological responders from non-responders. The grey dots highlight the four patients with a reported peritumoral inflammatory response.
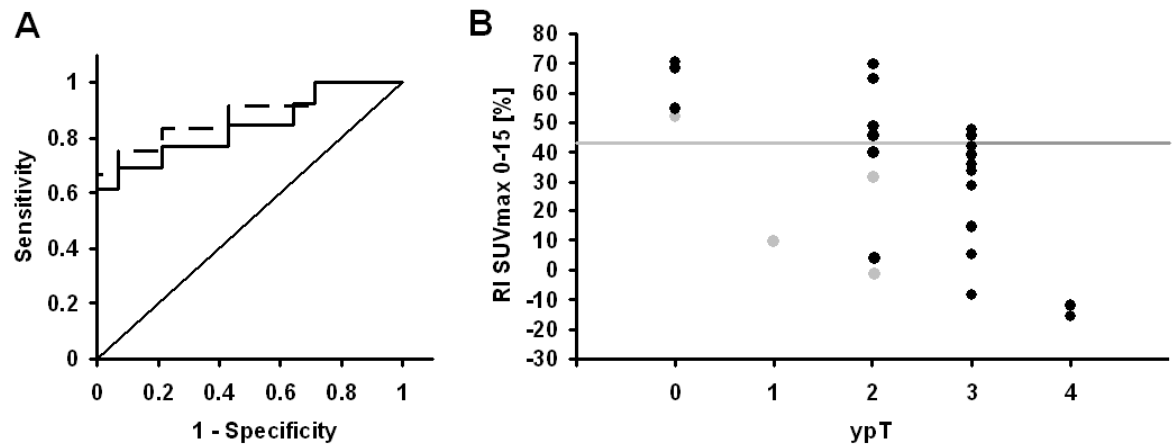

Figure 3.4: A) ROC-curves of the $S_{U} V_{\max }$ response-index on day 15 of the chemo-radiotherapy relative to the ypT-stage before (solid, AUC $=0.84$ ) and after (dashed, $A U C=0.89$ ) exlusion of the patients with a peritumoral inflammatory response. B) Response indices of the SUV $\mathrm{max}_{\max }$ on day 15 relative to the ypT-stage. The grey horizontal line indicates the ROC-curve analysis based cut-off value of $43 \%$ differentiating pathological responders from non-responders. The grey dots highlight the four patients with a peritumoral inflammatory response. 
Table 3.2: Average response index (RI) of the mean and maximum standardized uptake value (SUV) for pathological responders and non-responders, statistical significance and ROC-curve analysis for all PETcriteria revealing statistically significant different response indices for the two groups.

\begin{tabular}{|c|c|c|c|c|}
\hline & $\begin{array}{l}\text { Path. Resp. } \\
\text { (TRG 1, 2) }\end{array}$ & $\begin{array}{c}\text { Path. Non-Resp. } \\
\text { (TRG 3-5) }\end{array}$ & P-value & $\begin{array}{c}\text { ROC-curve } \\
\text { Area } \pm \text { Std. Error }\end{array}$ \\
\hline RI SUV mean 0-8 & $23.3 \pm 16.1 \%$ & $9.0 \pm 16.4 \%$ & 0.026 & $0.75 \pm 0.10$ \\
\hline RI SUV max 0-8 $_{\text {m }}$ & $29.5 \pm 17.7 \%$ & $9.4 \pm 18.0 \%$ & 0.013 & $0.78 \pm 0.09$ \\
\hline RI SUV mean 0-15 & $40.3 \pm 19.2 \%$ & $17.4 \pm 19.4 \%$ & 0.005 & $0.82 \pm 0.08$ \\
\hline RI SUV max 0-15 $_{\text {. }}$ & $47.2 \pm 22.0 \%$ & $17.9 \pm 21.6 \%$ & 0.001 & $0.87 \pm 0.07$ \\
\hline
\end{tabular}

\begin{tabular}{|c|c|c|c|c|}
\cline { 2 - 5 } \multicolumn{1}{c|}{} & $\begin{array}{c}\text { Path. Resp. } \\
\text { (ypT0, 1, 2) }\end{array}$ & $\begin{array}{c}\text { Path. Non-Resp. } \\
\text { (ypT3 ,4) }\end{array}$ & P-value & $\begin{array}{c}\text { ROC-curve } \\
\text { Area } \pm \text { Std. Error }\end{array}$ \\
\hline \hline RI SUV & $26.8 \pm 21.9 \%$ & $11.7 \pm 17.7 \%$ & 0.037 & $0.73 \pm 0.10$ \\
\hline \hline RI SUV & 26.8 & $21.4 \pm 18.9 \%$ & 0.007 & $0.81 \pm 0.09$ \\
\hline \hline RI SUV & $39.7 \pm 19.2 \%$ & $21.9 \pm 22.6 \%$ & 0.003 & $0.84 \pm 0.08$ \\
\hline
\end{tabular}

\subsection{Discussion}

This prospective study revealed a significant correlation between the metabolic tumor response, assessed with repeated FDG-PET-CT-imaging during CRT and the pathological tumor response. The best predictor of pathological tumor response turned out to be the RI of the SUV $V_{\max }$ calculated on day 15 of pre-operative CRT. Pre-surgical PETimaging, however did not reveal significantly different values for the evaluated PETcriteria when comparing pathological responding and non-responding malignancies.

Interestingly, four of the included patients presented with a (histopathologically reported) peritumoral inflammatory response which could be visually observed on the repeated PET-images. The increased FDG uptake associated with these inflammatory reactions resulted in mispredictions. Exclusion of the patients with a visually perceived peritumoral inflammatory response being apparent already early during CRT, from further analysis, improved the accuracy of used models for the prediction of both the TRG and the ypT-classification.

Previous reports on sequential PET-CT-imaging during pre-operative CRT for rectal cancer suggested further investigations on the best time point for the sequentially performed PET-CT image in order to find the the best predictive model for the pathological tumor response ${ }^{13,15}$. This study presents the first prospective study for LARC, in which multiple PET-CT scans during pre-operative CRT were undertaken in order to define the best optimal time-point for PET-imaging during therapy. In line with our findings, Cascini et al. also reported that early pathological response predictions, assessed from PET-imaging 12 days after the start of therapy, were superior to presurgical PET-based response predictions ${ }^{13}$. Interestingly, Wieder et al. showed comparable results for esophageal squamous cell carcinomas in two studies, also demonstrating that pre-surgical PET-measurements were less predictive for the pathological response compared to mid-treatment measurements ${ }^{20,21}$. In contrast to our findings, however Rosenberg et al. presented a study showing that pre-surgical PET-data were 
slightly superior compared to PET-imaging on day 14 of CRT for the prediction of the pathological treatment response in rectal cancer ${ }^{14}$.

Pre-surgical PET-images after a long course of CRT treatment however, mostly image smaller malignancies due to the significant downsizing, which can result in an underestimation of the FDG uptake within the malignancy due to the partial volume effect (PVE) ${ }^{22}$. Most of the patients included in our study demonstrated with only a small residual PET-positive tumor volume on the pre-surgical PET-CT scan due to a significant downsizing of the tumor, which was not the case early during CRT. Thus, for LARC, PET-images performed early during CRT are less influenced by the effects of PVE when compared to pre-surgical PET-imaging. The underestimation of the residual presurgical FDG uptake within the tumor due to the PVE might also explain the lack of correlation between $\mathrm{mCR}$ and $\mathrm{pCR}$ as found in our study.

Another important finding of this study and the study of Rosenberg et al. was the increased number of patients false negatively classified as pathological non-responders due to the accumulation of inflammatory cells in areas where tumor cells underwent necrosis ${ }^{14}$. Thus, an important confounder in the use of PET-imaging as a response predictor is a peritumoral inflammatory reaction. Inflammatory cells are known to avidly consume FDG ${ }^{23}$. An increased FDG uptake by inflammatory cells in the direct neighborhood of the tumor can lead to an underestimation of the decrease of FDG uptake within pathological responding malignancies, which could result in false negative classifications ${ }^{14}$. When applying sequential PET-imaging for the prediction of the pathological treatment response, tumors presenting with an increased peritumoral FDG uptake should be handled with care or even excluded from further response predictions. It would thus be helpful to be able to distinguish malignant lesions from inflammatory responses, which might help to further increase the accuracy of response predictions based on sequential PET-imaging. However, with the static PETimages performed in this study, no further differentiation was feasible. Dynamic and dual time point PET-imaging however might well be able to distinguish malignant from inflammatory tissue ${ }^{24,25}$.

Reliable FDG uptake quantifications should be ensured when using sequential PET$\mathrm{CT}$ imaging for the prediction of the treatment response ${ }^{15}$. Hindie et al. discussed the absolute necessity of imaging a malignancy at exactly the same time interval after FDG injection when using sequential PET-imaging because of the continuous FDG uptake during the first hours after FDG injection ${ }^{15}$. In contrast, however, most studies published involving the prediction of pathological treatment response with sequential PETimaging in rectal cancer based their analysis on FDG uptake times varying between 40 and 60 minutes $5,6,8,13,14$. Furthermore, strong fluctuations of the blood glucose level of the patient at the time of PET-imaging exist and might further influence the time-trend of the FDG uptake on sequential PET-images of the same patient ${ }^{15,16,26-28}$. None of the studies using sequential PET-imaging in rectal cancer presented to date performed a normalization of the PET-data for the measured blood glucose level. In our study, however all PET-data were normalized for the blood glucose level measured shortly before the FDG injection in order to minimize the possible influence of the fluctuating blood glucose level on the time trend of FDG uptake. Also, a strict time management with an exact time-interval of 60 minutes between FDG-administration and PETimaging was followed. 
An accurate prediction of the pathological tumor response already early during pre-operative treatment would enable for more individualized treatment regimens with as goal further improvement of the tumor response or a modified surgical approach. A reliable prediction of the final T-stage at the time of surgery with the help of the day 15 PET-CT would allow the surgeon to adapt the surgical approach to a less invasive technique, sparing the sphincter or even allow a TEM surgery with a laparatomy, which would significantly reduce morbidity, providing that other imaging modalities like MRI can assure an accurate prediction of a ypNO stage. In general, in attempts to define the right cut-off value for the FDG uptake decrease as a predictor for the pathological response, a high positive predictive value and a high specificity of the resulting prediction model should be preferred over a high negative predictive value and a high sensitivity in order to at least avoid a possible under-treatment rather than an over-treatment. However, the presented findings need to be validated in an independent dataset, before final conclusions can be drawn for the clinic.

In conclusion, the reduction of the SUV $\mathrm{max}_{\max }$ after 2 weeks of pre-operative CRT was found to be the best predictor for both the pathological tumor response (TRG) and the pathological T-stage (ypT). Thus, our data suggest that an accurate prediction of the TRG and the ypT is feasible already early during CRT with an AUC of 0.87 and 0.84 , respectively. 


\subsection{References}

[1] Kapiteijn E, Marijnen CA, Nagtegaal ID, et al. Preoperative radiotherapy combined with total mesorectal excision for resectable rectal cancer. N Engl J Med 2001;345:638-646.

[2] Sauer R, Becker H, Hohenberger W, et al. Preoperative versus postoperative chemoradiotherapy for rectal cancer. N Engl J Med 2004;351:1731-1740.

[3] Bosset JF, Collette L, Calais G, et al. Chemotherapy with preoperative radiotherapy in rectal cancer. N Engl J Med 2006;355:1114-1123.

[4] Valentini V, Coco C, Cellini N, et al. Ten years of preoperative chemoradiation for extraperitoneal T3 rectal cancer: acute toxicity, tumor response, and sphincter preservation in three consecutive studies. Int J Radiat Oncol Biol Phys 2001;51:371-383.

[5] Capirci C, Rampin L, Erba PA, et al. Sequential FDG-PET/CT reliably predicts response of locally advanced rectal cancer to neo-adjuvant chemo-radiation therapy. Eur J Nucl Med Mol Imaging 2007;34:1583-1593.

[6] Capirci C, Rubello D, Chierichetti F, et al. Long-term prognostic value of 18F-FDG PET in patients with locally advanced rectal cancer previously treated with neoadjuvant radiochemotherapy. AJR Am J Roentgenol 2006;187:W202-208.

[7] Vliegen RF, Beets-Tan RG, Vanhauten B, et al. Can an FDG-PET/CT Predict Tumor Clearance of the Mesorectal Fascia after Preoperative Chemoradiation of Locally Advanced Rectal Cancer? Strahlenther Onkol 2008;184:457-464.

[8] Kalff V, Duong C, Drummond EG, et al. Findings on 18F-FDG PET scans after neoadjuvant chemoradiation provides prognostic stratification in patients with locally advanced rectal carcinoma subsequently treated by radical surgery. J Nucl Med 2006;47:14-22.

[9] Amthauer H, Denecke T, Rau B, et al. Response prediction by FDG-PET after neoadjuvant radiochemotherapy and combined regional hyperthermia of rectal cancer: correlation with endorectal ultrasound and histopathology. Eur J Nucl Med Mol Imaging 2004;31:811-819.

[10] Denecke T, Rau B, Hoffmann KT, et al. Comparison of CT, MRI and FDG-PET in response prediction of patients with locally advanced rectal cancer after multimodal preoperative therapy: is there a benefit in using functional imaging? Eur Radiol 2005;15:1658-1666.

[11] Guillem JG, Moore HG, Akhurst T, et al. Sequential preoperative fluorodeoxyglucose-positron emission tomography assessment of response to preoperative chemoradiation: a means for determining longterm outcomes of rectal cancer. J Am Coll Surg 2004;199:1-7.

[12] Melton GB, Lavely WC, Jacene HA, et al. Efficacy of preoperative combined 18fluorodeoxyglucose positron emission tomography and computed tomography for assessing primary rectal cancer response to neoadjuvant therapy. J Gastrointest Surg 2007;11:961-969; discussion 969.

[13] Cascini GL, Avallone A, Delrio P, et al. 18F-FDG PET is an early predictor of pathologic tumor response to preoperative radiochemotherapy in locally advanced rectal cancer. J Nucl Med 2006;47:1241-1248.

[14] Rosenberg R, Herrmann K, Gertler R, et al. The predictive value of metabolic response to preoperative radiochemotherapy in locally advanced rectal cancer measured by PET/CT. Int J Colorectal Dis 2009;24:191-200.

[15] Hindie E, Hennequin C, Moretti JL. Predicting response to chemoradiotherapy in rectal and oesophageal cancer with 18F-FDG: prognostic value and possible role in patient management. Eur J Nucl Med Mol Imaging 2007;34:1576-1582.

[16] Beaulieu S, Kinahan P, Tseng J, et al. SUV varies with time after injection in (18)F-FDG PET of breast cancer: characterization and method to adjust for time differences. J Nucl Med 2003;44:1044-1050.

[17] Ollers M, Bosmans G, van Baardwijk A, et al. The integration of PET-CT scans from different hospitals into radiotherapy treatment planning. Radiother Oncol 2008;87:142-146.

[18] Daisne JF, Sibomana M, Bol A, et al. Tri-dimensional automatic segmentation of PET volumes based on measured source-to-background ratios: influence of reconstruction algorithms. Radiother Oncol 2003;69:247-250. 
[19] Mandard AM, Dalibard F, Mandard JC, et al. Pathologic assessment of tumor regression after preoperative chemoradiotherapy of esophageal carcinoma. Clinicopathologic correlations. Cancer 1994;73:2680-2686.

[20] Wieder HA, Brucher BL, Zimmermann F, et al. Time course of tumor metabolic activity during chemoradiotherapy of esophageal squamous cell carcinoma and response to treatment. J Clin Oncol 2004;22:900-908.

[21] Wieder HA, Ott K, Lordick F, et al. Prediction of tumor response by FDG-PET: comparison of the accuracy of single and sequential studies in patients with adenocarcinomas of the esophagogastric junction. Eur J Nucl Med Mol Imaging 2007;34:1925-1932.

[22] Soret M, Bacharach SL, Buvat I. Partial-volume effect in PET tumor imaging. J Nucl Med 2007;48:932-945.

[23] Kao PF, Chou YH, Lai CW. Diffuse FDG uptake in acute prostatitis. Clin Nucl Med 2008;33:308-310.

[24] Park H-H, Roh D-W, Lyu KY, et al. Usefulness of dynamic 18F-FDG PET scan in lung cancer and inflammation disease. J NUCL MED MEETING ABSTRACTS 2007;48:458P.

[25] Zhuang $\mathrm{H}$, Pourdehnad M, Lambright ES, et al. Dual time point 18F-FDG PET imaging for differentiating malignant from inflammatory processes. J Nucl Med 2001;42:1412-1417.

[26] Keyes JW, Jr. SUV: standard uptake or silly useless value? J Nucl Med 1995;36:1836-1839.

[27] Lindholm P, Minn H, Leskinen-Kallio S, et al. Influence of the blood glucose concentration on FDG uptake in cancer--a PET study. J Nucl Med 1993;34:1-6.

[28] Crippa F, Gavazzi C, Bozzetti F, et al. The influence of blood glucose levels on [18F]fluorodeoxyglucose (FDG) uptake in cancer: a PET study in liver metastases from colorectal carcinomas. Tumori 1997;83:748-752. 


\title{
Chapter
}

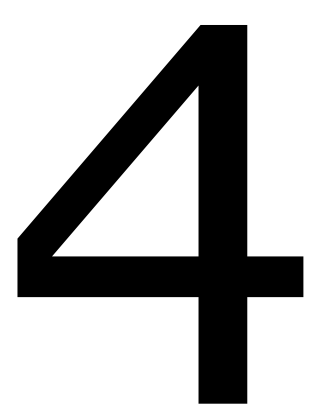

\section{Blood glucose level normalization and accurate timing improves the accuracy of PET-based treatment response predictions in rectal cancer.}

\author{
Marco Janssen \\ Michel Öllers \\ Ruud van Stiphout \\ Robert Riedl \\ Jørgen van den Bogaard \\ Jeroen Buijsen \\ Philippe Lambin \\ Guido Lammering
}

Radiotherapy \& Oncology, 2010; 95(2): 203-208 


\section{Abstract \\ Purpose}

To quantify the influence of fluctuating blood glucose level (BGLs) and the timing of FDG-PET acquisition on PET-based predictions of the pathological treatment response in rectal cancer.

\section{Materials \& Methods}

Thirty patients, diagnosed with locally-advanced-rectal-cancer (LARC), were included in this prospective study. Sequential FDG-PET-CT investigations were performed at four time points during and after pre-operative chemo-radiotherapy (CRT). All PET-data were normalized for the BGL measured shortly before FDG injection. The metabolic treatment response of the tumor was correlated with the pathological treatment response.

\section{Results}

During CRT, strong intra-patient BGL-fluctuations were observed, ranging from -38.7 to 95.6\%. BGL-normalization of the SUVs revealed differences ranging from -54.7 to $34.7 \%(p<0.001)$. Also, a SUV $\max$ time-dependency of $1.30 \pm 0.66$ every $10 \mathrm{~min}$. (range: 0.39 to 2.58 ) was found during the first $60 \mathrm{~min}$. of acquisition. When correlating the percent reduction of $\mathrm{SUV}_{\max }$ after 2 weeks of CRT with the pathological treatment response, a significant increase $(p=0.027)$ in the area under the curve of ROC-curve analysis was found when normalizing the PET-data for the measured BGLs, indicating an increase of the predictive strength.

\section{Conclusions}

This study strongly underlines the necessity of BGL-normalization of PET-data and a precise time-management between FDG-injection and the start of PET-acquisition when using sequential FDG-PET-CT imaging for the prediction of pathological treatment response. 


\subsection{Introduction}

Sequential fluorodeoxyglucose positron-emission-tomography computed-tomography (FDG-PET-CT) imaging has emerged as a promising method for the prediction of treatment response during or after neo-adjuvant chemo-radiotherapy (CRT) in an increasing number of malignancies ${ }^{1-17}$. For rectal cancer, the (percent) reduction of the maximum standardized-uptake-value $\left(\mathrm{SUV}_{\max }\right)$ within the tumor has been shown to predict the histo-pathological tumor response to pre-operative CRT ${ }^{1-6,9,12,14,15}$. An early prediction of the pathological treatment response of the tumor might in the future enable response-guided treatment algorithms on the basis of early changes of FDG uptake within the tumor $2,5,11,12,15$.

However, a diversity of factors is known to influence the results of PET-imaging ${ }^{5}$ 18-23. For PET-based predictions of the pathologic tumor response to pre-operative treatment, it is important to make sure that no or as little as possible fluctuations occur for the factors known to influence the results of sequential PET-imaging ${ }^{5}$. Especially the time interval between FDG injection and the start of PET acquisition as well as the patient's blood glucose level (BGL) at the time of PET-imaging strongly influence the SUVs ${ }^{5,18-23}$. Despite the known advantage of delayed FDG-PET-imaging for the detection of malignant lesions, the majority of studies published involving sequential PET-imaging in rectal cancer started the PET acquisition within the first 60 min after FDG injection ${ }^{1-4,12}$. Hamberg et al. stated that if SUV measurements are performed at the plateau, more robust predictions of treatment response could be achieved $^{20}$.

Moreover, it was found that the BGL of the patient at the time of PET-imaging inverse proportionally affects FDG uptake ${ }^{18,22,23}$. However, until now, most of the studies published on sequentially performed FDG-PET-images in rectal cancer did not pay attention to intra-patient BGL fluctuations or the absence of a plateau for FDG uptake when analyzing the acquired PET-data ${ }^{1-4,7-9,12}$. Although the concept correcting PET-data for BGL fluctuations is not entirely new, the application and importance of it for the evaluation of treatment response using sequential FDG-PET-imaging is often overlooked, especially by the radiation oncology community.

Thus, we felt that it is of particular importance to investigate and quantify the influence of intra-patient BGL-fluctuations on sequentially acquired PET-scans and the resulting prediction of pathological treatment response as well as the influence of fluctuations in the time interval between FDG injection and the start of PET-acquisition of rectal cancer patients. 


\subsection{Materials \& Methods}

\section{Patient Characteristics}

Thirty patients diagnosed with non-metastasized locally-advanced-rectal-cancer (LARC) were included in this study from which the clinical TN staging was evaluated on a pretreatment magnetic resonance (MR) scan (Table 4.1). All patients were referred to preoperative treatment with radiotherapy (28 fractions of $1.8 \mathrm{~Gy}$ daily) and concomitant chemotherapy (Capecitabine $825 \mathrm{mg} / \mathrm{m}^{2}$ BID), followed by a total mesorectal excision (TME). According to the Dutch law, the medical ethics committee approved the trial. All patients gave written informed consent before entering the study.

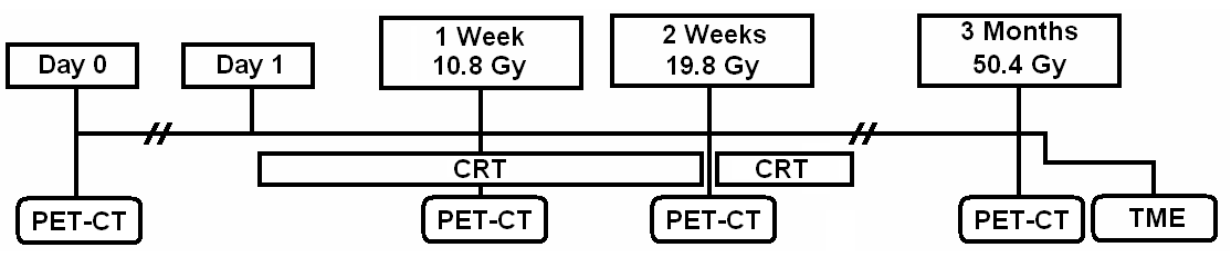

Figure 4.1: Study scheme for the assessment of the metabolic treatment response for rectal cancer patients referred to pre-operative treatment with neo-adjuvant chemo-radiotherapy (CRT), followed by a total mesorectal excision (TME).

\section{PET-CT Acquisition and Processing}

As a part of the study, all patients underwent sequential FDG-PET-CT imaging at four different time points: prior to therapy, at the end of the first and second week of CRT and once shortly before surgery (Fig. 4.1). All PET-CT scans were performed by use of a dedicated Siemens Biograph 40 TruePoint PET-CT scanner (Siemens Medical, Erlangen, Germany) with an axial field of $16.2 \mathrm{~cm}$, slice thickness of $3 \mathrm{~mm}$ and a pixel spacing of $5.3466 \mathrm{~mm}$ in both directions. The scanner is equipped with ulta-fast detector electronics (Pico 3D) and has a spatial resolution of approximately $6 \mathrm{~mm}$ full-width-at-halfmaximum (FWHM). PET data acquisition was done 3 dimensional (3D), requiring a proper scatter correction. CT-based attenuation and decay correction was performed. PET-images were reconstructed from the acquired list-mode (LM) data using Fourierrebinning (FORE) and ordered-subset-expectation-maximization-reconstruction (OSEM 2D) with 4 iterations and 8 subsets. The patients were positioned equal to the radiotherapy treatment position using a laser alignment system to have minimal variations between imaging and treatment conditions and between the different imaging timepoints. For each scan, first a topogram was made from which the radiation oncologist manually selected the region of interest (ROI) for the dynamic PET-scan.

Next, a CT-scan of the ROI was acquired, after which the FDG, with the activity depending on the weight of the patient (weight $[\mathrm{kg}] * 4+20[\mathrm{MBq}]$ ), was injected intravenously followed by injection of physiologic-saline $(10 \mathrm{ml})$. Acquisition of the dynamic PET-scan over 60 minutes was started directly after FDG administration. The list-mode PET-data were rebinned and reconstructed using 28 time-frames (10x30 sec., $5 \times 60$ sec., $5 \times 120 \mathrm{sec}$. and $8 \times 300 \mathrm{sec}$.) ${ }^{24}$. Additionally, the dynamic PET-data were corrected for tumor motion during dynamic acquisition, using the Image-Fusion-toolbox of the PMOD software package (PMOD Technologies Ltd., Zurich, Switzerland). Due to tech- 
nical problems or patient incompliance, not all PET-CT scans could be performed as planned. For one patient no FDG could be injected for the PET-CT scan after one week of CRT, whereas two more patients refused PET-CT imaging after the second week of CRT. For six of the included patients, no PET-CT scan could be performed prior to surgery.

\section{Blood Glucose Levels}

All patients were instructed to fast for at least 6 hours prior to FDG injection. Prior to each FDG injection, the patient's BGL was measured using an automatic device (LifeScan One Touch Ultra, LifeScan Inc., Milpitas, USA) ${ }^{25}$. All acquired PET-data were normalized for the BGL using the following equation:

$S U V_{\text {normalized }}=S U V \cdot \frac{[G l u]}{100}$, with [Glu] the measured BGL $[\mathrm{mg} / \mathrm{dl}]{ }^{21,26}$.

\section{PET Analysis}

For all PET-scans, the tumor was delineated using automated SUV-thresholding with the threshold (percentage of SUV $_{\max }$ within the tumor) depending on the tumor-tobackground signal ratio (SBR-method), with the gluteus muscle selected as relevant background ${ }^{27,28}$. Dedicated software (TrueD VC50, Siemens Medical, Erlangen, Germany) was used for the quantification of FDG uptake within the tumor. Subsequently, response indices (RIs), indicating the percentage reduction of the FDG uptake relative to the pre-treatment measured value, were calculated for all 3 follow-up FDG-PET-CT scans. If no residual metabolic activity was present on the pre-surgical PET-scan, the patient was classified as a metabolic complete responder (mCR) and SUV $\mathrm{max}_{\max }$ and the RI were set to respectively 0 and $100 \%$ for the pre-surgical PET-data.

\section{PET Acquisition Timing}

To study the time dependency of SUV $\mathrm{max}_{\max }$ determinations within the tumor, a tumor contour was generated on the last time frame of the dynamic PET-data using dedicated software (PMOD Technologies Ltd., Zurich, Switzerland). The maximum time activity curve (TAC) of the tumor was visually inspected for the presence of a decrease in the FDG uptake rate during the first $60 \mathrm{~min}$. after FDG injection indicating saturation for FDG uptake. For each of the included patients, the time dependencies of the $S V_{\max }$ determination were calculated over the last 8 time-frames (40 min.) of the maximum TAC and expressed as the difference in SUV $_{\max }$ resulting from a $10 \mathrm{~min}$. time discrepancy between FDG injection and PET acquisition. Ten of the included patients underwent two additional static PET-CT scans prior to the start of therapy after FDG uptake periods of respectively 90 and 120 min.. The maximum TAC resulting from dynamic PET-acquisition as well as the SUVs $s_{\max }$ calculated from the two additional static PETscans were fitted into combined TACs over $120 \mathrm{~min}$. 


\section{Pathological Tumor Response}

After the TME, the pathological treatment response within the tumor was evaluated by determination of the tumor-regression-grade (TRG) according to the Mandardcriteria: TRG1, complete tumor response; TRG2, residual cancer cells scattered through fibrosis; TRG3, an increased number of residual cancer cells, with predominant fibrosis; TRG4, residual cancer outgrowing fibrosis; TRG5, no regressive changes within the tumor $^{29}$. All tumors were retrospectively classified by an experienced pathologist (RR), who was blinded for the PET-data. Based on the TRGs, the tumors were grouped into two groups, respectively pathological responders (TRG1, 2) and non-responders (TRG 3-5).

\section{Statistical Analysis}

Statistical analyses were performed using SPSS (version 15.0; SPSS Inc., Chicago, IL, USA). Comparisons of related measurements were performed using a Wilcoxon-signed rank test, whereas a Mann-Whitney $U$ test was used in case of independent samples. Differences were considered to be significant when the $p$-value was less than 0.05 . To study the influence of BGL-fluctuations on PET-based predictions of pathological treatment response, the RI of SUV $\mathrm{Sax}_{\text {, }}$ both before and after BGL-normalization were correlated to the pathological treatment response using receiver-operatingcharacteristics (ROC) analysis. A z-test was used to study the statistical significance of the difference between the ROC-curves before and after BGL-normalization ${ }^{30}$.

\subsection{Results}

\section{Intra-Patient BGL-fluctuations}

For the included patients, large intra-patient BGL-fluctuations were observed, ranging from -38.7 to $95.6 \%$ (average: $5.2 \pm 20.9 \%$ ) when compared to the pre-treatment measured BGL (Table 4.1). BGL-normalization of the sequential PET-data resulted in an average increase of $S U V_{\max }$ of $9.7 \pm 14.1 \%$ (range: -54.7 to $34.7 \%$ ) when comparing the maximum SUVs before and after BGL-normalization $(p<0.001)$ (Table 4.2). Large individual differences were noticed in the magnitude of the intra-patient BGL fluctuations, leading to remarkable changes in the time trends of FDG uptake when applying BGLnormalization for some patients, whereas for other patients almost no fluctuations of the BGL were observed (Table $4.1 \& 4.2$ ).

\section{SUV Time Sensitivity}

Visual analysis of the maximum TACs of the tumor revealed that in none of the investigated tumors a plateau for FDG uptake occurred within the first $60 \mathrm{~min}$. after FDG injection. Importantly, the determination of the $S_{U} V_{\max }$ within the first 60 min. after FDG injection emerged as very time sensitive leading to large variations in the values with an average difference of $1.30 \pm 0.66$ in only $10 \mathrm{~min}$. (range: 0.39 to 2.58 $\Delta S U V_{\max } / 10 \mathrm{~min}$.). Only one of the investigated tumors reached a plateau for FDG uptake within the first 120 min. after FDG injection (Fig. 4.2). 
However, overall the FDG uptake rate started to decrease after an uptake period of 90 min., resulting in a statistically significant reduction of the SUV $\mathrm{Vax}_{\max }$ time sensitivity by $56.3 \pm 30.6 \%$ (range: 2.5 to $95.0 \%$ ) ( $p=0.005$ ) when compared to the first $60 \mathrm{~min}$. after FDG injection (Fig. 4.2).
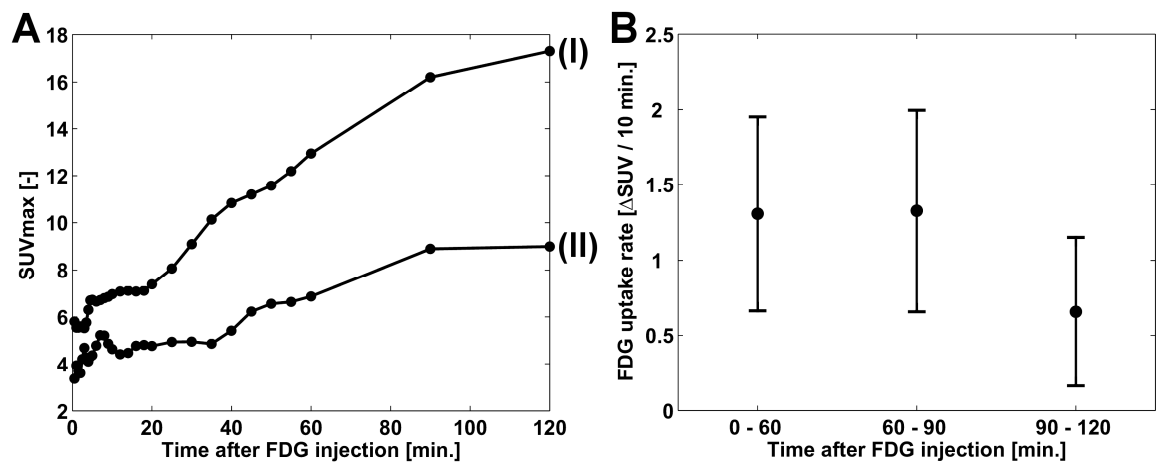

Figure 4.2: (A) Maximum time activity curves of the tumor for the first 2 hours after FDG injection for both a representative patient (I) and a patient showing a plateau for FDG uptake (II). (B) Overall, the FDG uptake rate within the tumor decreased after an uptake period of 90 minutes, resulting in a statistically significant reduction of the SUV time sensitivity $(p=0.005)$.

\section{Metabolic and pathological response evaluation}

When quantifying the FDG uptake within the tumor, for each patient the highest FDG uptake was detected on the pre-treatment PET-CT scan, followed by a statistically significant reduction of SUV $_{\max }$ during pre-operative CRT. From the pathological response evaluation, thirteen of the included patients (43\%) were classified as pathological responders (4 TRG 1, 9 TRG 2), whereas seventeen patients (57\%) were classified as pathological non-responders (11 TRG 3, 6 TRG 4) (Table 4.1).

\section{Correlation between the metabolic and pathological treatment response}

When correlating the RIs of SUV $V_{\max }$ both before and after BGL-normalization to the pathological treatment response, statistically significant higher RIs of SUV max $_{\text {were }}$ found for the pathological responders after two weeks of CRT before BGLnormalization and after both one and two weeks of CRT after BGL-normalization (Fig. 4.3). When looking at the resulting $p$-values, it can be concluded that normalizing the sequential PET-data for the BGL of the patient at the time of PET-imaging improves the statistical significance of the difference between the RIs of SUV max $_{\text {ax }}$ of pathological responders and non-responders (Fig. 4.3). ROC-curve analysis of the RI of SUV $\max$ after 2 weeks of pre-operative CRT presented an increase of the area under the curve (AUC) of the ROC-curve, from $0.749 \pm 0.097$ to $0.867 \pm 0.074(p=0.027)$, by performing a BGLnormalization of the PET-data, which proves an statistically significant increase of the accuracy of the prediction of pathological treatment response based on the reduction

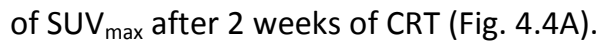




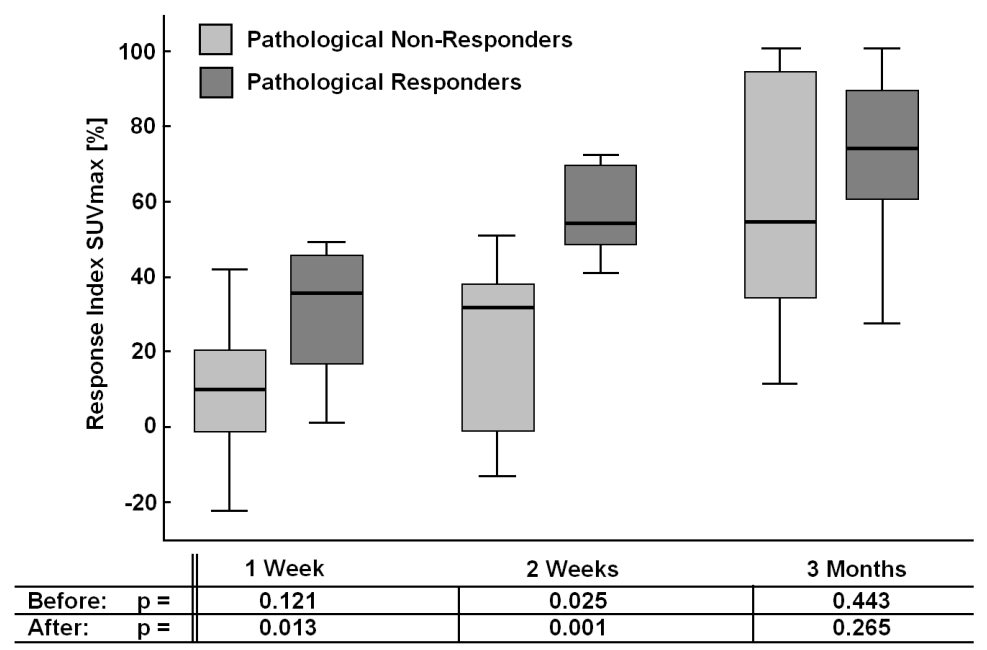

Figure 4.3: Boxplots of the response indices of $\mathrm{SUV}_{\max }$ measured during and after pre-operative treatment for pathological non-responders (TRG 3-5)(light boxes) and pathological responders (TRG 1-2) (dark boxes). The $p$-values indicate the statistical significance of the differences in response index between pathological responders and non-responders both before and after normalization of the sequential PET-data for the patients' blood glucose level.
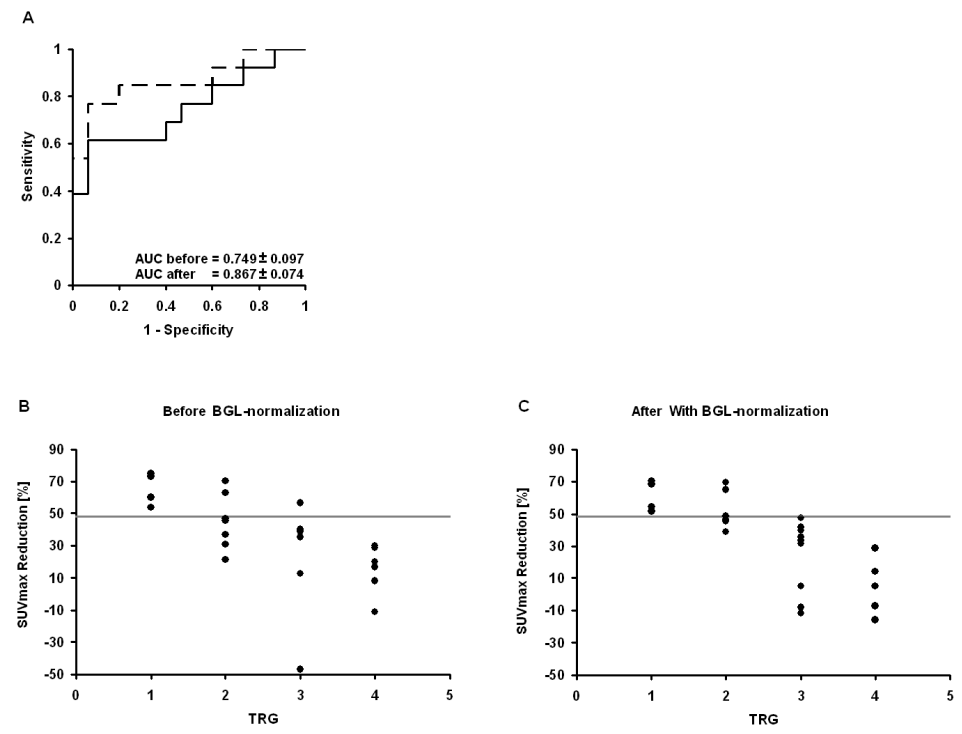

Figure 4.4: A) Receiver Operating Characteristics (ROC) curves for the percent reduction of SUV $\max$ at the end of the second week of pre-operative treatment with chemo-radiotherapy both before (solid) and after

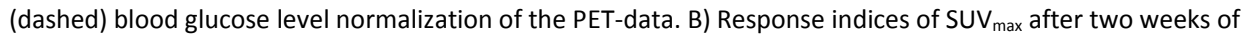
CRT-treatment relative to the tumor regression grade (TRG) with the ROC-curve based cut-off value of $48 \%$ for the differentiation of pathological responders (TRG 1-2) from non-responders (TRG 3-5). C) Response indices of BGL-normalized values of SUV $V_{\max }$ relative to the TRG with the same ROC-curve based cut-off value of $48 \%$ to differentiate between responders and non-responders. 
Also, when plotting the RIs of SUV $\max$ relative to the TRG's, less overlap between the RIs of the pathological responders and non-responders were found after BGLnormalization of the PET-data when compared to the situation before normalization of the PET-data (Fig. 4.4B, C).

Table 4.1: Overview of the patient characteristics, clinical staging (cTNM), the measured blood glucose levels (BGLs) and the tumor regression grade (TRG).

\begin{tabular}{|c|c|c|c|c|c|c|c|c|}
\hline $\begin{array}{l}\text { Pat. } \\
\text { Nr. }\end{array}$ & $\begin{array}{c}\text { Weight } \\
{[\mathrm{kg}]}\end{array}$ & $\begin{array}{c}\text { Age } \\
\text { [years] }\end{array}$ & cTNM & $\begin{array}{c}\text { BGL } \\
{[\mathrm{mmol} / \mathrm{I}]} \\
\text { pre- } \\
\text { treatment }\end{array}$ & $\begin{array}{c}\text { BGL } \\
{[\mathrm{mmol} / \mathrm{l}]} \\
1 \\
\text { week } \\
\end{array}$ & $\begin{array}{c}\text { BGL } \\
{[\mathrm{mmol} / \mathrm{l}]} \\
2 \\
\text { weeks } \\
\end{array}$ & $\begin{array}{c}\text { BGL } \\
\text { [mmol/I] } \\
\text { pre- } \\
\text { surgical }\end{array}$ & TRG \\
\hline 1 & 75 & 48 & T3N1M0 & 5.7 & 6.4 & & 6.4 & 3 \\
\hline 2 & 86 & 64 & T2N1M0 & 6.2 & & 6.5 & & 1 \\
\hline 3 & 60 & 70 & T3N2M0 & 5.9 & 6.2 & 5.8 & 6.3 & 3 \\
\hline 4 & 103 & 57 & T3N2M0 & 5.2 & 5.2 & 5.4 & & 2 \\
\hline 5 & 64 & 78 & T3N2M0 & 6.1 & 5.9 & & 5.2 & 3 \\
\hline 6 & 70 & 71 & T4N2M0 & 6.4 & 5.6 & 5.7 & 6.2 & 2 \\
\hline 7 & 79 & 83 & T3N1M0 & 6.7 & 4.5 & 6.4 & 6.3 & 2 \\
\hline 8 & 83 & 60 & T3N2M0 & 5.5 & 5.3 & 5.8 & 4.9 & 2 \\
\hline 9 & 75 & 71 & T3N2M0 & 6.3 & 6.9 & 6.7 & 6.6 & 3 \\
\hline 10 & 84 & 66 & T3N1M0 & 10.6 & 7.9 & 8.1 & 6.5 & 3 \\
\hline 11 & 70 & 71 & T3N2MO & 5.9 & 6.6 & 7.1 & 6.7 & 3 \\
\hline 12 & 69 & 60 & T3N2M0 & 6.4 & 5.4 & 6.0 & 6.7 & 4 \\
\hline 13 & 70 & 73 & T3N1M0 & 5.4 & 5.6 & 6.4 & & 1 \\
\hline 14 & 84 & 64 & T3N2M0 & 6.3 & 6.1 & 6.4 & 5.5 & 4 \\
\hline 15 & 102 & 54 & T3N1M0 & 5.4 & 5.3 & 5.3 & 5.3 & 3 \\
\hline 16 & 55 & 45 & T3NOMO & 5.3 & 5.9 & 5.8 & 5.8 & 3 \\
\hline 17 & 62 & 61 & T3N2M0 & 5.3 & 5.1 & 5.0 & & 3 \\
\hline 18 & 79 & 69 & T3N1M0 & 6.4 & 6.6 & 6.7 & & 2 \\
\hline 19 & 90 & 71 & T3N2M0 & 5.4 & 5.9 & 5.9 & 6.3 & 3 \\
\hline 20 & 88 & 70 & T3N2M0 & 6.1 & 6.4 & 6.1 & 7.8 & 4 \\
\hline 21 & 69 & 55 & T3N2M0 & 7.2 & 8.7 & 8.3 & 7.1 & 1 \\
\hline 22 & 75 & 74 & T3N2M0 & 6.2 & 6.8 & 6.2 & 5.5 & 2 \\
\hline 23 & 68 & 71 & T3NOMO & 5.7 & 5.9 & 6.5 & 6.6 & 4 \\
\hline 24 & 83 & 69 & T3N2M1 & 6.8 & 11.8 & 12.2 & 13.3 & 3 \\
\hline 25 & 52 & 77 & T4N1M0 & 5.3 & 7.6 & 7.7 & 6.8 & 4 \\
\hline 26 & 79 & 73 & T3NOMO & 5.2 & 5.4 & 5.7 & 4.9 & 2 \\
\hline 27 & 70 & 76 & T3NOMO & 5.7 & 6.2 & 6.7 & 5.9 & 1 \\
\hline 28 & 84 & 66 & T3N2M0 & 6.2 & 6.7 & 6.4 & 6.1 & 2 \\
\hline 29 & 98 & 52 & T3N2M0 & 8.6 & 6.5 & 5.8 & & 2 \\
\hline 30 & 110 & 43 & T3N1M0 & 6.3 & 6.1 & 6.1 & 6.7 & 4 \\
\hline
\end{tabular}


Table 4.2: Overview of the maximum FDG uptake (SUV $\mathrm{max}_{\text {) }}$ ) within the tumor at all four PET-CT imaging timepoints before (pre) and after (post) normalization for the patients blood glucose level measured shortly before FDG injection.

\begin{tabular}{|c|c|c|c|c|c|c|c|c|}
\hline \multirow[t]{2}{*}{ Pat. Nr. } & \multicolumn{2}{|c|}{$\begin{array}{c}\text { SUV }_{\max } \\
\text { pre-treatm. [-] }\end{array}$} & \multicolumn{2}{|c|}{$\begin{array}{c}\text { SUV }_{\max } \\
1 \text { week [-] }\end{array}$} & \multicolumn{2}{|c|}{$\begin{array}{c}\text { SUV }_{\text {max }} \\
2 \text { weeks [-] }\end{array}$} & \multicolumn{2}{|c|}{$\begin{array}{c}\text { SUV }_{\max } \\
\text { pre-surgical [-] }\end{array}$} \\
\hline & pre & post & pre & post & pre & post & pre & post \\
\hline 1 & 15.6 & 16.0 & 9.2 & 10.6 & & & 10.1 & 11.6 \\
\hline 2 & 11.9 & 13.3 & & & 5.5 & 6.4 & & \\
\hline 3 & 12.4 & 13.2 & 12.6 & 14.1 & 7.4 & 7.7 & 0.0 & 0.0 \\
\hline 4 & 20.9 & 19.6 & 10.9 & 10.2 & 6.2 & 6.0 & & \\
\hline 5 & 15.3 & 15.6 & 11.2 & 10.3 & & & 6.2 & 5.9 \\
\hline 6 & 12.5 & 14.4 & 10.5 & 10.6 & 8.6 & 8.8 & 6.5 & 6.5 \\
\hline 7 & 23.3 & 28.1 & 20.9 & 16.9 & 8.6 & 9.9 & 0.0 & 0.0 \\
\hline 8 & 8.4 & 8.3 & 8.5 & 8.1 & 8.1 & 8.5 & 6.9 & 6.1 \\
\hline 9 & 12.6 & 14.3 & 9.9 & 12.3 & 8.1 & 9.8 & 8.1 & 8.3 \\
\hline 10 & 8.8 & 16.9 & 10.9 & 15.5 & 12.9 & 18.9 & 6.6 & 7.8 \\
\hline 11 & 19.6 & 20.8 & 10.1 & 12.0 & 8.5 & 10.9 & 4.2 & 5.1 \\
\hline 12 & 9.6 & 11.1 & 10.2 & 10.0 & 8.8 & 9.5 & 7.3 & 8.8 \\
\hline 13 & 20.9 & 20.3 & 10.8 & 10.9 & 5.2 & 6.0 & & \\
\hline 14 & 11.0 & 12.5 & 8.9 & 9.8 & 7.7 & 8.9 & 6.6 & 6.5 \\
\hline 15 & 26.7 & 26.7 & 23.8 & 23.8 & 16.1 & 16.1 & 0.0 & 0.0 \\
\hline 16 & 7.8 & 7.4 & 8.5 & 9.0 & 6.8 & 7.1 & 3.4 & 4.0 \\
\hline 17 & 28.9 & 27.6 & 29.8 & 27.4 & 17.7 & 17.7 & & \\
\hline 18 & 13.4 & 15.5 & 13 & 15.5 & 11.6 & 14.0 & & \\
\hline 19 & 15.8 & 15.2 & 13.3 & 14.1 & 9.5 & 10.1 & 0.0 & 0.0 \\
\hline 20 & 16.6 & 18.2 & 12.6 & 14.5 & 11.8 & 13.0 & 8.6 & 12.1 \\
\hline 21 & 20.2 & 26.2 & 12.7 & 19.9 & 8.0 & 11.9 & 5.2 & 5.7 \\
\hline 22 & 13.8 & 15.4 & 11.8 & 14.4 & 7.5 & 8.4 & 4.3 & 4.3 \\
\hline 23 & 11.2 & 11.5 & 11.9 & 12.7 & 9.3 & 10.9 & 4.4 & 5.2 \\
\hline 24 & 8.0 & 9.8 & 5.5 & 11.7 & 4.8 & 10.6 & 0.0 & 0.0 \\
\hline 25 & 10.6 & 10.2 & 7.4 & 10.1 & 8.5 & 11.8 & 7.5 & 9.1 \\
\hline 26 & 15.5 & 14.2 & 9.9 & 8.1 & 9.8 & 7.3 & 4.1 & 3.6 \\
\hline 27 & 17.2 & 17.7 & 8.6 & 9.6 & 4.6 & 5.6 & 0.0 & 0.0 \\
\hline 28 & 21.4 & 23.9 & 12.2 & 14.7 & 11.3 & 13.0 & 7.6 & 8.4 \\
\hline 29 & 15.9 & 24.6 & 14.6 & 17.1 & 12.5 & 13.1 & & \\
\hline 30 & 9.9 & 11.2 & 8.8 & 9.7 & 11.0 & 12.0 & 6.2 & 7.4 \\
\hline
\end{tabular}




\subsection{Discussion}

Over the last years, sequential FDG-PET-CT imaging has been shown to be of promise for the prediction of pathological treatment response in an increasing number of malignancies $1-3,5-13,15,16$. However, a diversity of factors are known to influence the results of PET-imaging, for instance the used equipment and protocol, uptake time of the FDG and the patients BGL at the time of PET-imaging ${ }^{5,19,20}$. When performing sequential FDG-PET-CT imaging within a single patient, especially when using the SBRmethod for tumor delineation, it is of high importance to use the same PET-CT scanner and imaging protocol for each of the examinations ${ }^{27,28}$.

Analysis of the sequentially acquired PET-data without taking into account a BGLnormalization could, in the case of large intra-patient BGL-fluctuations, result in incorrect SUV time-trends, which again could lead to an incorrect prediction of the treatment response. However, in earlier published literature, a clear trend was presented between the FDG uptake within the tumor and the BGL of the patients prior to FDG injection, enabling normalization of PET-data for the BGL of the patient measured shortly before FDG injection ${ }^{22,23}$.To the best of our knowledge, none of the published studies, except two, regarding treatment response predictions for rectal cancer based on sequential PET-imaging accounted for the BGL when evaluating the acquired PETdata ${ }^{10,15}$. For the patients included in our study, the SUVs normalized for the BGL were found to be statistically significant different from the SUVs before BGL-normalization, this in contrast to the findings of Stahl et al. ${ }^{10}$. Also, performing a normalization of the PET-data for the measured BGLs resulted in an increased AUC of ROC-curve analysis and a decreased $p$-value when predicting the pathological treatment response based on the percent reduction of SUV $\mathrm{V}_{\max }$ after two weeks of CRT. The significantly increased AUC of the ROC-curve after BGL-normalization as well as the decrease in overlap between responders and non-responders prove that performing a BGL-normalization for sequential PET-data improves the accuracy of the prediction of pathological treatment response. Within this study, BGL measurements were performed using an automatic device as we did not include venous blood-sampling, which would enable clinical and analytical BGL measurements. However, the used automatic device (LifeScan One Touch, LifeScan Inc., Milpitas, USA) was proven to be the most accurate device, resulting in $100 \%$ of the measurements in the clinically accurate range for blood-glucose levels above $3.89 \mathrm{mmol} / \mathrm{I}^{25}$. As can be concluded from this study, BGL normalization improves the accuracy of PET-based response predictions. However, when using PETbased response prediction models in clinical practice in the near future, patients presenting with large BGL fluctuations or patients known to suffer from diabetes mellitus should be handled with care or even be excluded from further analysis to avoid erroneous response-guided modifications of the treatment protocol.

Another important finding of this study is the fact that the time interval between FDG injection and the start of PET acquisition in sequential FDG-PET-CT scans needs to be very consistent with the initially performed FDG-PET-CT scan, as FDG continues to show uptake by the tumor at high rates even 60 min. after FDG injection. The determination of a SUV before saturation of FDG uptake within the tumor results in substantial SUV-variations if time differences between FDG injection and start of PET acquisition occur ${ }^{5,19,20}$. Although this SUV time sensitivity is the highest in the first $90 \mathrm{~min}$. after 
FDG injection, most protocols published involving the prediction of treatment response based on sequential PET imaging in rectal cancer used uptake periods ranging from 40 to 60 minutes ${ }^{1-4,12}$. This might result in substantial variations in SUV measurements leading to misinterpretations in pathological response predictions. Delayed PET acquisition after $90 \mathrm{~min}$. however significantly reduces this SUV time dependency. Therefore, an accurate time-management between FDG injection and the start of PET acquisition with a consistent time interval for all sequentially performed FDG-PET-CT scans or a delayed start of the PET acquisition after $90 \mathrm{~min}$. is needed in order to minimize the time-dependency of the acquired SUV changes.

In conclusion, this study underlines the necessity for a strict protocol for sequential FDG-PET-CT imaging of rectal cancer patients, in which the blood glucose level should be determined and normalized for and consistent time intervals between FDG injection and the start of PET-imaging should be followed. Otherwise, strategies to differentiate between pathological responding and non-responding malignancies on the basis of absolute cut-off values for the (percent) reduction of FDG uptake should be handled with care. 


\subsection{References}

[1] Kalff, V, Duong, C, Drummond, EG, Matthews, JP, Hicks, RJ. Findings on 18F-FDG PET scans after neoadjuvant chemoradiation provides prognostic stratification in patients with locally advanced rectal carcinoma subsequently treated by radical surgery. J Nucl Med 2006;47:14-22.

[2] Cascini, GL, Avallone, A, Delrio, P, et al. 18F-FDG PET is an early predictor of pathologic tumor response to preoperative radiochemotherapy in locally advanced rectal cancer. J Nucl Med 2006;47:1241-1248.

[3] Capirci, C, Rampin, L, Erba, PA, et al. Sequential FDG-PET/CT reliably predicts response of locally advanced rectal cancer to neo-adjuvant chemo-radiation therapy. Eur J Nucl Med Mol Imaging 2007;34:1583-1593.

[4] Capirci, C, Rubello, D, Chierichetti, F, et al. Long-term prognostic value of 18F-FDG PET in patients with locally advanced rectal cancer previously treated with neoadjuvant radiochemotherapy. AJR Am J Roentgenol 2006;187:W202-208.

[5] Hindie, E, Hennequin, C, Moretti, JL. Predicting response to chemoradiotherapy in rectal and oesophageal cancer with 18F-FDG: prognostic value and possible role in patient management. Eur J Nucl Med Mol Imaging 2007;34:1576-1582.

[6] Amthauer, H, Denecke, T, Rau, B, et al. Response prediction by FDG-PET after neoadjuvant radiochemotherapy and combined regional hyperthermia of rectal cancer: correlation with endorectal ultrasound and histopathology. Eur J Nucl Med Mol Imaging 2004;31:811-819.

[7] Denecke, T, Rau, B, Hoffmann, KT, et al. Comparison of CT, MRI and FDG-PET in response prediction of patients with locally advanced rectal cancer after multimodal preoperative therapy: is there a benefit in using functional imaging? Eur Radiol 2005;15:1658-1666.

[8] Guillem, JG, Moore, HG, Akhurst, T, et al. Sequential preoperative fluorodeoxyglucose-positron emission tomography assessment of response to preoperative chemoradiation: a means for determining longterm outcomes of rectal cancer. J Am Coll Surg 2004;199:1-7.

[9] Melton, GB, Lavely, WC, Jacene, HA, et al. Efficacy of preoperative combined 18fluorodeoxyglucose positron emission tomography and computed tomography for assessing primary rectal cancer response to neoadjuvant therapy. J Gastrointest Surg 2007;11:961-969; discussion 969.

[10] Stahl, A, Ott, K, Schwaiger, M, Weber, WA. Comparison of different SUV-based methods for monitoring cytotoxic therapy with FDG PET. Eur J Nucl Med Mol Imaging 2004;31:1471-1478.

[11] Wieder, HA, Ott, K, Lordick, F, et al. Prediction of tumor response by FDG-PET: comparison of the accuracy of single and sequential studies in patients with adenocarcinomas of the esophagogastric junction. Eur J Nucl Med Mol Imaging 2007;34:1925-1932.

[12] Rosenberg, R, Herrmann, K, Gertler, R, et al. The predictive value of metabolic response to preoperative radiochemotherapy in locally advanced rectal cancer measured by PET/CT. Int J Colorectal Dis 2009;24:191-200.

[13] van Baardwijk, A, Bosmans, G, Dekker, A, et al. Time trends in the maximal uptake of FDG on PET scan during thoracic radiotherapy. A prospective study in locally advanced non-small cell lung cancer (NSCLC) patients. Radiother Oncol 2007;82:145-152.

[14] Vliegen, RF, Beets-Tan, RG, Vanhauten, B, et al. Can an FDG-PET/CT predict tumor clearance of the mesorectal fascia after preoperative chemoradiation of locally advanced rectal cancer? Strahlenther Onkol 2008;184:457-464.

[15] Janssen, MH, Ollers, MC, Riedl, RG, et al. Accurate Prediction of Pathological Rectal Tumor Response after Two Weeks of Preoperative Radiochemotherapy Using (18)F-FluorodeoxyglucosePositron Emission Tomography-Computed Tomography Imaging. Int J Radiat Oncol Biol Phys 2010; 77: 392-399.

[16] Young, H, Baum, R, Cremerius, U, et al. Measurement of clinical and subclinical tumour response using [18F]-fluorodeoxyglucose and positron emission tomography: review and 1999 EORTC recommendations. European Organization for Research and Treatment of Cancer (EORTC) PET Study Group. Eur J Cancer 1999;35:1773-1782.

[17] Monteil, J, Mahmoudi, N, Leobon, S, et al. Chemotherapy response evaluation in metastatic colorectal cancer with FDG PET/CT and CT scans. Anticancer Res 2009;29:2563-2568. 
[18] Crippa, F, Gavazzi, C, Bozzetti, F, et al. The influence of blood glucose levels on [18F]fluorodeoxyglucose (FDG) uptake in cancer: a PET study in liver metastases from colorectal carcinomas. Tumori 1997;83:748-752.

[19] Keyes, JW, Jr. SUV: standard uptake or silly useless value? J Nucl Med 1995;36:1836-1839.

[20] Hamberg, LM, Hunter, GJ, Alpert, NM, Choi, NC, Babich, JW, Fischman, AJ. The dose uptake ratio as an index of glucose metabolism: useful parameter or oversimplification? J Nucl Med 1994;35:1308-1312.

[21] Beaulieu, S, Kinahan, P, Tseng, J, et al. SUV varies with time after injection in (18)F-FDG PET of breast cancer: characterization and method to adjust for time differences. J Nucl Med 2003;44:1044-1050.

[22] Langen, KJ, Braun, U, Rota Kops, E, et al. The influence of plasma glucose levels on fluorine-18fluorodeoxyglucose uptake in bronchial carcinomas. J Nucl Med 1993;34:355-359.

[23] Lindholm, P, Minn, H, Leskinen-Kallio, S, Bergman, J, Ruotsalainen, U, Joensuu, H. Influence of the blood glucose concentration on FDG uptake in cancer--a PET study. J Nucl Med 1993;34:1-6.

[24] Janssen, MH, Aerts, HJ, Ollers, MC, et al. Tumor delineation based on time-activity curve differences assessed with dynamic fluorodeoxyglucose positron emission tomography-computed tomography in rectal cancer patients. Int J Radiat Oncol Biol Phys 2009;73:456-465.

[25] Brunner, GA, Ellmerer, M, Sendlhofer, G, et al. Validation of home blood glucose meters with respect to clinical and analytical approaches. Diabetes Care 1998;21:585-590.

[26] Eary, JF, Mankoff, DA. Tumor metabolic rates in sarcoma using FDG PET. J Nucl Med 1998;39:250254.

[27] Daisne, JF, Sibomana, M, Bol, A, Doumont, T, Lonneux, M, Gregoire, V. Tri-dimensional automatic segmentation of PET volumes based on measured source-to-background ratios: influence of reconstruction algorithms. Radiother Oncol 2003;69:247-250.

[28] Ollers, M, Bosmans, G, van Baardwijk, A, et al. The integration of PET-CT scans from different hospitals into radiotherapy treatment planning. Radiother Oncol 2008;87:142-146.

[29] Mandard, AM, Dalibard, F, Mandard, JC, et al. Pathologic assessment of tumor regression after preoperative chemoradiotherapy of esophageal carcinoma. Clinicopathologic correlations. Cancer 1994;73:2680-2686.

[30] Hanley, JA, McNeil, BJ. A method of comparing the areas under receiver operating characteristic curves derived from the same cases. Radiology 1983;148:839-843. 


\section{Chapter}

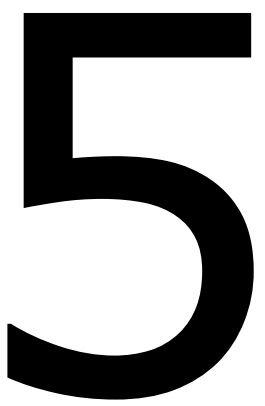

\section{PET-based treatment response evaluation in rectal cancer: prediction and validation.}

Marco Janssen

Michel Öllers

Ruud van Stiphout

Robert Riedl

Jørgen van den Bogaard

Jeroen Buijsen

Philippe Lambin

Guido Lammering

International Journal of Radiation Oncology * Biology * Physics, 2010; Accepted for publication 


\section{Abstract \\ Purpose}

To develop a PET-based response prediction model to differentiate pathological responders from non-responders. The predictive strength of the model was validated in a second patient group, treated and imaged identical to the patients on which the predictive model was based.

\section{Materials \& Methods}

Fifty-one rectal cancer patients were prospectively included in this study. All patients underwent FDG-PET-CT-imaging both prior to the start of chemo-radiotherapy (CRT) and after two weeks of treatment. Pre-operative treatment with CRT was followed by a total mesorectal excision. From the resected specimen, the tumor regression grade (TRG) was scored according to the Mandard criteria. From one patient group $(\mathrm{N}=30)$, the metabolic treatment response was correlated with the pathological treatment response, resulting in a ROC-curve based cut-off value for the reduction of SUV $\mathrm{max}_{\operatorname{ma}}$ within the tumor to differentiate pathological responders (TRG 1-2) from nonresponders (TRG 3-5). The applicability of the selected cut-off value for new patients was validated in a second patient group $(\mathrm{N}=21)$.

\section{Results}

When correlating the metabolic and pathological treatment response for the first patient group using ROC-curve analysis ( $A U C=0.98$ ), a cut-off value of $48 \% \mathrm{SUV}_{\max }$ reduction was selected to differentiate pathological responders from non-responders (specificity of $100 \%$, sensitivity of $64 \%$ ). Applying this cut-off value to the second patient group resulted in a specificity and sensitivity of respectively $93 \%$ and $83 \%$, with only one of the pathological non-responders being false positively predicted as pathological responding.

\section{Conclusions}

For rectal cancer, an accurate PET-based prediction of the pathological treatment response is feasible already after two weeks of chemo-radiotherapy. The presented predictive model could be used to select patients to be considered for less invasive surgical interventions or even a "wait and see policy". Also, based on the predicted response, early modifications of the treatment protocol are possible, which might result in an improved clinical outcome. 


\subsection{Introduction}

Over the last years, the reduction of the metabolic activity of rectal tumors, assessed with repeated fluorodeoxyglucose posistron-emission-tomography computedtomography (FDG-PET-CT) imaging, during pre-operative treatment has been shown to accurately predict the pathological treatment response ${ }^{1-14}$. Most of the published studies about PET-based treatment response predictions determined a (ROC-curve based) cut-off value, percent reduction of the mean or maximum standardized-uptakevalue (SUV) within the tumor, after finishing pre-operative treatment, to differentiate pathological responders from non-responders. However, also early metabolic treatment responses within the tumor, as early as two weeks after the start of preoperative treatment, were presented as a strong predictor of the pathological treatment response ${ }^{9,10,12}$. Two studies even presented early PET-based response predictions as being more accurate when compared to response predictions based on preand post-treatment PET-imaging ${ }^{9,12}$. A prediction of the pathological treatment response early during pre-operative treatment is more attractive for clinical practice, as this enables individualized treatment schemes in the near future, possibly resulting in an improved tumor control or modified surgical approaches like less invasive or delayed surgery in combination with an intensive imaging follow-up.

The main objective of a PET-based predictive model is the actual prediction of the pathological treatment response for patients not included in the patient group on which the model is based. However, so far, none of the presented PET-based response predictive models was yet validated with a secondary patient group. As for further development and clinical usefulness of PET-based response predictive models a proper validation with a secondary patient group is required, this study was undertaken to develop a PET-based prediction model to differentiate pathological responders from non-responders. The predictive strength of the presented predictive model was validated in a second patient group, treated and imaged identical to the patients on which the predictive model was based.

\subsection{Materials \& Methods}

\section{Patient Characteristics}

Fifty-one patients diagnosed with locally-advanced-rectal-cancer (LARC) were included in this study, from which the clinical TN staging was evaluated on a pre-treatment magnetic resonance (MR) scan (Table 5.1). All patients were pre-operatively treated with radiotherapy ( 28 fractions of $1.8 \mathrm{~Gy}, 5$ fractions/week) and concomitant chemotherapy (Capecitabine $825 \mathrm{mg} / \mathrm{m}^{2}$ BID, 7 days a week), followed by a total mesorectal excision (TME) approximately 3 months after the start of pre-operative treatment (Fig. 5.1). Radiotherapy treatment was delivered by 4 beams, AP, PA and left and right lateral, each with an energy of 10MV. For each patient, a 3D conformal plan was made according to the ICRU specifications. 
As a part of the study, all patients underwent FDG-PET-CT imaging both prior to the start of CRT and at the end of the second week of treatment (Fig. 5.1). According to the Dutch law, the Medical Ethics Committee approved the trial. All patients gave written informed consent before entering the study.

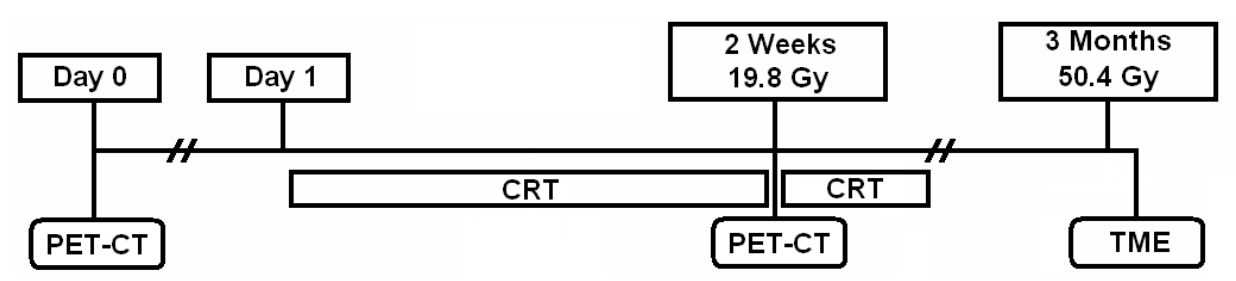

Figure 5.1: Study scheme for the assessment of early metabolic response during pre-operative treatment with chemo-radiotherapy (CRT). All included patients underwent FDG-PET-CT imaging at two time points: prior to the start of treatment and two weeks after the onset of treatment.

\section{PET-CT Imaging and Processing}

All PET-CT scans were performed using a dedicated Siemens Biograph 40 TruePoint PET-CT simulator (Siemens Medical, Erlangen, Germany) with an axial field of view of $16.2 \mathrm{~cm}$, slice thickness of $3 \mathrm{~mm}$ and a pixel spacing of $5.3456 \mathrm{~mm}$ in both directions. The scanner is equipped with ultra-fast detector electronics (Pico3D) and has a spatial resolution of approximately $6 \mathrm{~mm}$ full-width-at-half-maximum (FWHM). PET-imaging was performed in 3D, requiring a proper scatter correction. CT-based attenuation and decay correction was performed. PET images were reconstructed from the acquired list-mode (LM) data using Fourier-rebinning (FORE) and ordered-subset-expectationmaximization-reconstruction (OSEM 2D) with 4 iterations and 8 subsets. After a fasting period of at least 6 hours, FDG was injected intravenously, with the activity normalized for the weight of the patient (weight $[\mathrm{kg}] * 4+20[\mathrm{MBq}]$ ). After an uptake period of 60 minutes, PET acquisition was started with the patient positioned equal to the radiotherapy treatment position using a movable laser alignment system. Additionally, all PETdata were normalized for the blood glucose level measured shortly before FDG administration $^{15}$.

\section{PET Analysis}

For each PET-scan, the tumor was automatically delineated using SUV-thresholding with the threshold (percentage of SUV $\mathrm{max}_{\operatorname{mit}}$ win the tumor) depending on the tumorto-background signal ratio with the gluteus muscle selected as relevant background ${ }^{16}$, 17. Dedicated software (TrueD, Siemens Medical, Erlangen, Germany) was used to calculate the maximum FDG uptake $\left(S_{U V} V_{\max }\right)$ within the tumor. Subsequently, a response index $(\mathrm{RI})$, indicating the percentage reduction relative to the pre-treatment measured value, was calculated. 


\section{Pathological Tumor Response}

For each tumor, the pathological treatment response was evaluated by determination of the tumor-regression-grade (TRG) as proposed by Mandard ${ }^{18}$. All tumors were retrospectively classified by an experienced pathologist (RR), who was blinded for the PET-data, as follows: TRG1, complete tumor response; TRG2, residual cancer cells scattered through fibrosis; TRG3, an increased number of residual cancer cells, with predominant fibrosis; TRG4, residual cancer outgrowing fibrosis; TRG5, no regressive changes within the tumor. Based on the TRGs, the patients were grouped into pathological responders (TRG1, 2) and non-responders (TRG 3-5).

\section{Response Prediction and Validation}

For thirty of the included patients, the metabolic and pathological treatment responses were correlated using ROC-curve analysis. From the ROC-curve, a cut-off value for the percent reduction of SUV $_{\max }$ within the tumor after two weeks of chemoradiotherapy (CRT) treatment was selected to differentiate pathological responders from non-responders. When selecting this cut-off value, a high specificity was preferred over a high sensitivity to avoid pathological non-responders from being false positively predicted as pathological responders, resulting in possible under-treatment of pathological non-responding patients. Next, the applicability of the selected cut-off value was validated for new patients $(\mathrm{N}=21)$, imaged and treated under identical conditions as the patients on which the predictive model was based.

\section{Statistical Analysis}

Statistical analyses were performed using SPSS (version 15.0; SPSS Inc., Chicago, IL, USA). Comparisons of related measurements were performed using a Wilcoxon-signed rank test and receiver operating characteristics (ROC) analysis was performed to evaluate the optimal cut-off value of $S V_{\max }$ reduction to differentiate pathological responders from non-responders.

\subsection{Results}

\section{Peritumoral Inflammatory Responses}

From the first patient group $(\mathrm{N}=30)$, four patients presented with a peritumoral inflammatory response, visually observed from the PET-scan performed at the end of the second week of treatment. Also for the second patient group $(\mathrm{N}=21)$, used for validation of the predictive model, one patient presented with a peritumoral inflammatory response. As inflammatory cells are known to avidly consume glucose, all patients with a peritumoral inflammatory response were excluded from further analysis to prevent an underestimation of the metabolic treatment response of the tumor. When delineating the tumor using automatic SUV-thresholding, an increase of the PET-positive tissue volume was found after two weeks of CRT treatment for the above mentioned patients (Fig. 5.2). As an increase of the volume of the malignancy is not to be expected during pre-operative CRT treatment, the increase of the PET-positive tissue volume is a clear indication of a peritumoral inflammatory response. 
Also, for these patients, a more diffuse FDG uptake was observed after two weeks of treatment with a decreased tumor to background signal ratio resulting in a less clear PET-based distinction between malignant and non-malignant tissue (Fig. 5.2). All visually observed peritumoral inflammatory responses after two weeks of treatment were confirmed after pathological examination of the resected specimen.

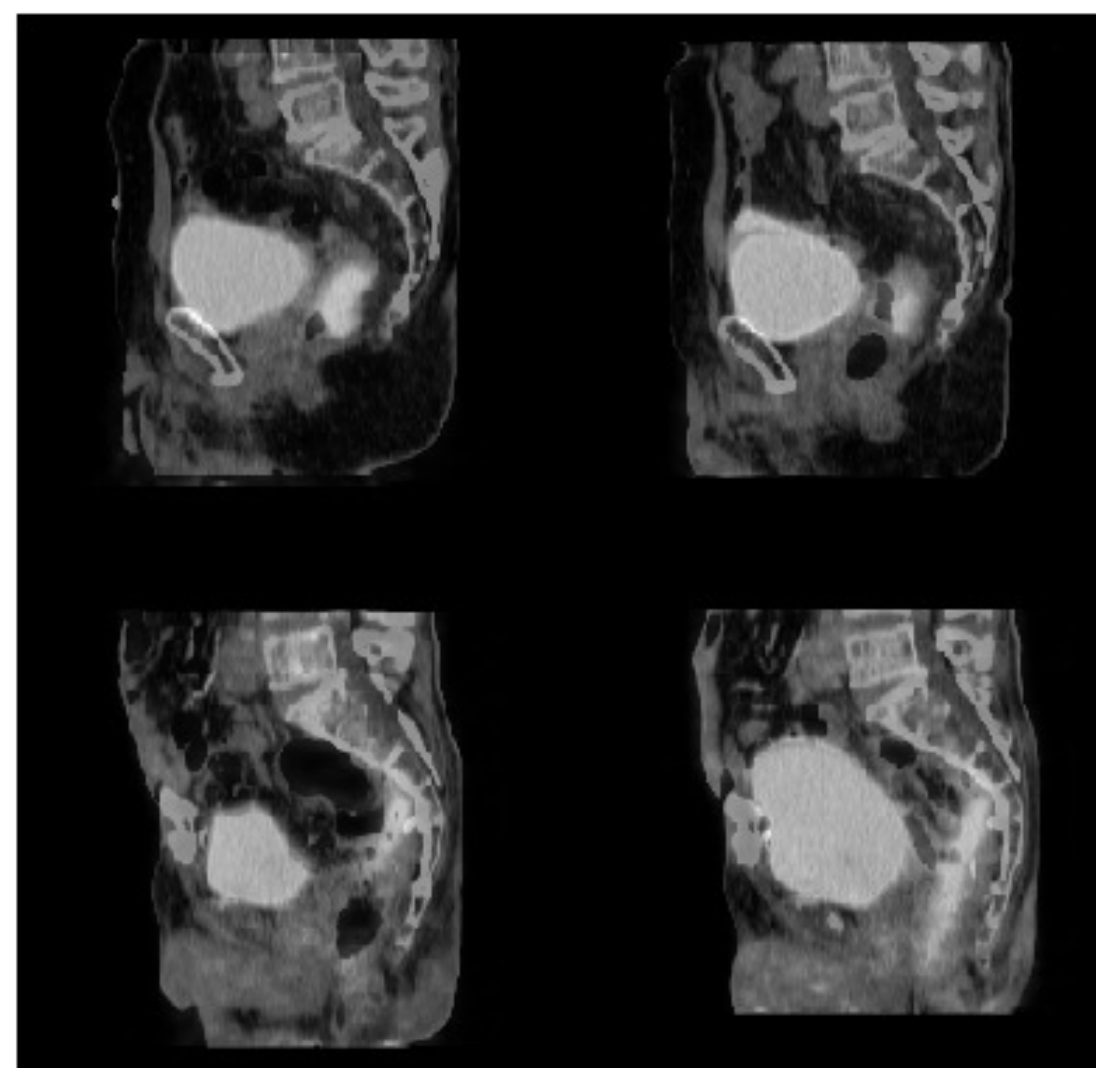

Figure 5.2: FDG-PET-CT images at both PET-CT imaging time points for respectively a representative patient (upper row) and a patient presenting with a (pathological reported) peritumoral inflammatory response (lower row).

\section{Response Prediction}

For the first patient group, an average reduction of $S U V_{\max }$ within the tumor of $33.6 \pm 25.8 \%(p<0.001)$ was observed after two weeks of CRT (Fig. 5.3 \& Table 5.1). The $\mathrm{SUV}_{\text {max }}$ reduction within the tumor was correlated with the pathological response by ROC-curve analysis, resulting in an area under the curve (AUC) of 0.98 (Table 5.2). From the resulting ROC-curve, a cut-off value of $48 \%$ SUV $_{\max }$ reduction was selected to differentiate pathological responders from non-responders, resulting in a specificity of $100 \%$ to prevent pathological non-responders from being false positively predicted as 
pathological responder (Fig. 5.4). However, for this cut-off value, a sensitivity of $64 \%$ was found, with 4 pathological responding patients to be false negatively predicted as pathological non-responding (Fig. 5.4).

\section{Validation}

Also for the second patient group, a significant reduction of SUV $\mathrm{max}_{\max }$ within the tumor (32.3 $\pm 27.0 \%, p=0.001)$ was found after two weeks of CRT (Fig. 5.3 \& Table 5.1). When applying the ROC-curve based cut-off value of $48 \%$ to differentiate pathological responders from non-responders, a specificity and sensitivity of respectively $93 \%$ and $83 \%$ was found, with only one of the pathological non-responding patients (TRG 3) being false-positively predicted as pathological responding, whereas one pathological responder (TRG 1) was false negatively predicted as being a pathological non-responder (Fig. 5.4).

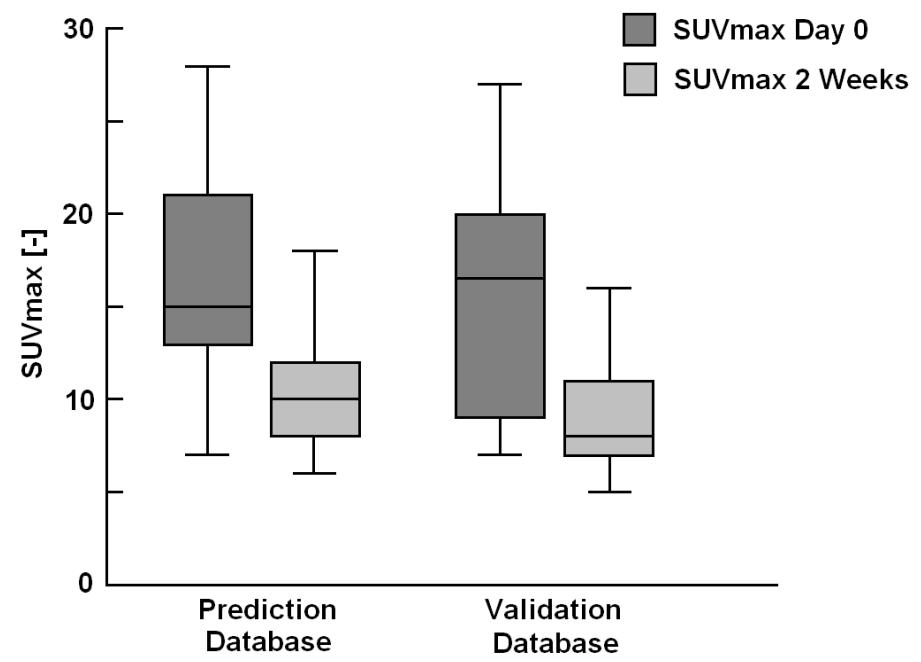

Figure 5.3: Boxplots of SUV $\mathrm{max}_{\text {mith }}$ win the tumor at both PET-CT imaging time points for both patient groups, with the dark and light grey boxes presenting respectively the pre-treatment SUV $V_{\max }$ within the tumor and the SUV $V_{\max }$ after two weeks of chemo-radiotherapy treatment. 
Table 5.1: Overview of the clinical staging (cTNM), the tumor regression grade (TRG) and the response index (RI) of the maximum standardized uptake value $\left(S_{U} V_{\max }\right)$ within the tumor after two weeks of pre-operative chemo-radiotherapy treatment for the prediction patient group $(\mathrm{N}=26)$ and the validation patient group ( $N=20)$.

\begin{tabular}{|c|c|c|c|c|c|c|c|}
\hline $\begin{array}{l}\text { Pat. } \\
\text { Nr. }\end{array}$ & cTNM & TRG & $\begin{array}{c}\text { RI SUV } \\
\text { [\%] }\end{array}$ & $\begin{array}{l}\text { Pat. } \\
\text { Nr. }\end{array}$ & cTNM & TRG & $\begin{array}{c}\text { RI SUV } \text { max }_{\text {max }} \\
\text { [\%] }\end{array}$ \\
\hline 1 & T2N1M0 & 1 & 51,9 & 1 & T4N1M0 & 3 & 47,6 \\
\hline 2 & T3N2M0 & 3 & 41,7 & 2 & T3N1M0 & 3 & $-9,2$ \\
\hline 3 & T3N2M0 & 2 & 69,4 & 3 & T3NOMO & 1 & 62,5 \\
\hline 4 & T4N2M0 & 2 & 38,9 & 4 & T4N1M0 & 4 & 16,9 \\
\hline 5 & T3N1M0 & 2 & 64,8 & 5 & T3N2M0 & 1 & 69,1 \\
\hline 6 & T3N2M0 & 3 & 31,5 & 6 & T3NOMO & 4 & $-19,9$ \\
\hline 7 & T3N1M0 & 3 & $-11,8$ & 7 & T3N2MO & 4 & 2,9 \\
\hline 8 & T3N2M0 & 3 & 47,6 & 8 & T2N1M0 & 2 & 55,9 \\
\hline 9 & T3N2M0 & 4 & 14,4 & 9 & T3N2MO & 4 & 10,6 \\
\hline 10 & T3N1M0 & 1 & 70,4 & 10 & T3N1M0 & 3 & 54,4 \\
\hline 11 & T3N2M0 & 4 & 28,8 & 11 & T3N1M0 & 1 & 45,3 \\
\hline 12 & T3N1M0 & 3 & 40,8 & 12 & T3N2M0 & 3 & 40,0 \\
\hline 13 & T3NOMO & 3 & 4,1 & 13 & T3N1M0 & 4 & 15,0 \\
\hline 14 & T3N2M0 & 3 & 35,9 & 14 & T3N2M0 & 4 & 45,1 \\
\hline 15 & T3N2M0 & 3 & 33,6 & 15 & T2NOMO & 3 & 1,2 \\
\hline 16 & T3N2M0 & 4 & 28,6 & 16 & T3N2M0 & 2 & 63,2 \\
\hline 17 & T3N2M0 & 1 & 54,6 & 17 & T3NOMO & 3 & 37,7 \\
\hline 18 & T3N2M0 & 2 & 45,5 & 18 & T3NOMO & 3 & 44,0 \\
\hline 19 & T3NOMO & 4 & 5,2 & 19 & T3N1M0 & 1 & 53.1 \\
\hline 20 & T3N2M1 & 3 & $-8,2$ & 20 & T4NOMO & 3 & 40,3 \\
\hline 21 & T4N1M0 & 4 & $-15,7$ & & & & \\
\hline 22 & T3NOMO & 2 & 48,6 & & & & \\
\hline 23 & T3NOMO & 1 & 68,4 & & & & \\
\hline 24 & T3N2M0 & 2 & 45,6 & & & & \\
\hline 25 & T3N2M0 & 2 & 46,7 & & & & \\
\hline 26 & T3N1M0 & 4 & $-7,1$ & & & & \\
\hline
\end{tabular}


Prediction Database

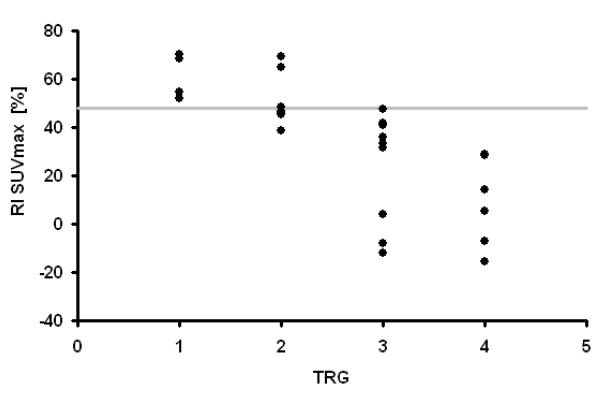

Validation Database

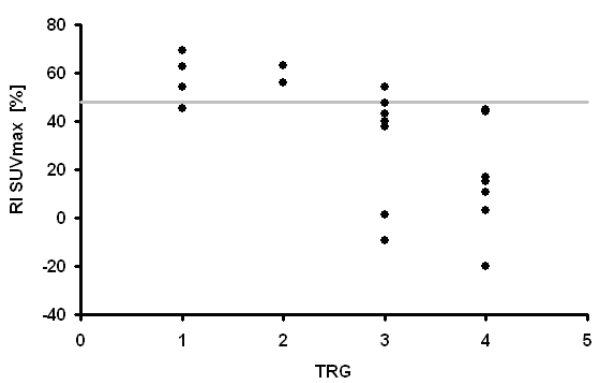

Figure 5.4: Response indices (RI) of SUV $\mathrm{max}_{\max }$ after two weeks of pre-operative chemo-radiotherapy treatment relative to the tumor-regression-grade (TRG) for both the prediction (left) and validation (right) database. The grey horizontal line indicates the ROC-curve based cut-off value of $48 \% \mathrm{SUV}_{\max }$ reduction for the differentiation of pathological responders (TRG 1-2) from non-responders (TRG 3-5).

Table 5.2: Overview of average metabolic responses (RI SUV $V_{\max }$ ) as assessed with FDG-PET-imaging, relative to the tumor regression grades (TRGs).

\begin{tabular}{|c|c|}
\cline { 2 - 2 } \multicolumn{1}{c|}{} & \multicolumn{1}{c|}{ RI SUV $_{\max }$} \\
\hline \hline TRG 1 & $59.4 \pm 9.4 \%$, range: 45.3 to $70.4 \%$ \\
\hline \hline TRG 2 & $53.2 \pm 10.5 \%$, range: 38.9 to $69.4 \%$ \\
\hline \hline TRG 3 & $26.7 \pm 22.9 \%$, range: -11.8 to $54.4 \%$ \\
\hline TRG 4 & $13.0 \pm 20.4 \%$, range: -19.9 to $45.1 \%$ \\
\hline
\end{tabular}

\begin{tabular}{|c|c|}
\cline { 2 - 2 } \multicolumn{1}{c|}{} & \multicolumn{2}{c|}{ RI SUV $_{\max }$} \\
\hline \hline TRG 1-2 & $56.1 \pm 10.2 \%$, range: 38.9 to $70.4 \%$ \\
\hline TRG 3-5 & $20.5 \pm 22.5 \%$, range: -19.9 to $54.4 \%$ \\
\hline
\end{tabular}

\subsection{Discussion}

Response predictive models based on changes of the metabolic activity of the tumor, assessed with repeated FDG-PET-CT imaging, were presented to result in accurate predictions of the pathological treatment response ${ }^{1-14}$. However, proper validation of published PET-based response predictive models has not yet been performed. This is the first study performing a validation of a PET-based response prediction model using a SUV cut-off value to differentiate pathological responders from non-responders. Validation of such response predictive models is required to ensure whether the presented model is applicable on patients who are not included in the patient group on which the model is based.

When using a PET-based response prediction model for the differentiation of pathological responders and non-responders, the cut-off value (percent reduction of the FDG uptake within the tumor) used to differentiate responders from non-responders should be chosen in such a way that as less non-responding patients as possible are false positively predicted as pathological responding ${ }^{12}$. 
This, to avoid under-treatment of false positively predicted pathological nonresponders when performing modifications of the treatment protocol based on the predicted treatment response. The selected ROC-curve based cut-off value of $48 \%$ SUV $_{\text {max }}$ reduction at the end of the second week of pre-operative CRT (sensitivity 64\%, specificity $100 \%$ ) was applied on a second patient group, resulting in a sensitivity and specificity of respectively $83 \%$ and $93 \%$, with one pathological non-responder being false positively predicted as pathological responding. From these results, it was concluded that a PET-based predictive model using a cut-off value (percent reduction of SUV $_{\max }$ within the tumor) can be used to accurately predict the pathological treatment response for patients not included in the patient group on which the predictive model is based.

For this study, we defined patients with a tumor regression grade (TRG) of 1 or 2 according to the Mandard-criteria as being pathological responders and patients with a TRG 3-5 as pathological non-responders. Earlier published literature proved patients with a TRG of 1-2 to have a better prognosis compared to patients with a TRG of 3-5 ${ }^{19}$, ${ }^{20}$. Patients presenting with a TRG 1 or 2 were proven to have less chance on local failure, whereas they have an improved chance on metastasis- and disease free survival as well as overall survival ${ }^{20}$. Also, an extended time interval between pre-operative (C)RT treatment and surgery has been presented to result in more pronounced tumor regression and downstaging, whereas a shorter time interval may interrupt ongoing of tumor necrosis ${ }^{21-23}$. We believe that a PET-based response predictive model as presented in this manuscript could in the near future be helpful to identify those TRG 1-2 patients in order to improve the tumor response by including these patients in a boost trial and/or apply an extended time interval between RT and surgery.

For some of the patients included in this study, a peritumoral inflammatory response was visually observed from the PET-images acquired after the second week of CRT. As inflammatory cells are known to avidly consume glucose(analogs), peritumoral inflammatory responses can lead to an underestimation of the metabolic response of the tumor, ultimately resulting in false negative predictions of pathological responders $10,12,24$. Patients presenting with a (visually observed) peritumoral inflammatory response should not be included in the patient group on which a PET-based response predictive model is based and such PET-based response predictive model should not be applied for patient with a peritumoral inflammatory response.

Importantly, when predicting the pathological treatment response based on sequential PET-data, standardization of the used PET-imaging protocol concerning the PET image reconstruction algorithm, injected FDG activities and uptake periods, SUV calculation method, blood glucose level measurements and correction of the PET-data for the blood glucose level is required ${ }^{11,16,25-28}$.

In conclusion, this is the first validation of a PET-based model for the prediction of the pathological treatment response. The presented results prove that an accurate prediction of the pathological treatment response based on the reduction of SUV $V_{\max }$ is possible already after two weeks of CRT treatment for patients treated and imaged identical to the patients on which the response predictive model is based. 


\subsection{References}

[1] Capirci C, Rampin L, Erba PA, et al. Sequential FDG-PET/CT reliably predicts response of locally advanced rectal cancer to neo-adjuvant chemo-radiation therapy. Eur J Nucl Med Mol Imaging 2007;34:1583-1593.

[2] Capirci C, Rubello D, Chierichetti F, et al. Long-term prognostic value of 18F-FDG PET in patients with locally advanced rectal cancer previously treated with neoadjuvant radiochemotherapy. AJR Am J Roentgenol 2006;187:W202-208.

[3] Vliegen RF, Beets-Tan RG, Vanhauten B, et al. Can an FDG-PET/CT predict tumor clearance of the mesorectal fascia after preoperative chemoradiation of locally advanced rectal cancer? Strahlenther Onkol 2008;184:457-464.

[4] Kalff V, Duong C, Drummond EG, et al. Findings on 18F-FDG PET scans after neoadjuvant chemoradiation provides prognostic stratification in patients with locally advanced rectal carcinoma subsequently treated by radical surgery. J Nucl Med 2006;47:14-22.

[5] Amthauer H, Denecke T, Rau B, et al. Response prediction by FDG-PET after neoadjuvant radiochemotherapy and combined regional hyperthermia of rectal cancer: correlation with endorectal ultrasound and histopathology. Eur J Nucl Med Mol Imaging 2004;31:811-819.

[6] Denecke T, Rau B, Hoffmann KT, et al. Comparison of CT, MRI and FDG-PET in response prediction of patients with locally advanced rectal cancer after multimodal preoperative therapy: is there a benefit in using functional imaging? Eur Radiol 2005;15:1658-1666.

[7] Guillem JG, Moore HG, Akhurst T, et al. Sequential preoperative fluorodeoxyglucose-positron emission tomography assessment of response to preoperative chemoradiation: a means for determining longterm outcomes of rectal cancer. J Am Coll Surg 2004;199:1-7.

[8] Melton GB, Lavely WC, Jacene HA, et al. Efficacy of preoperative combined 18fluorodeoxyglucose positron emission tomography and computed tomography for assessing primary rectal cancer response to neoadjuvant therapy. J Gastrointest Surg 2007;11:961-969; discussion 969.

[9] Cascini GL, Avallone A, Delrio P, et al. 18F-FDG PET is an early predictor of pathologic tumor response to preoperative radiochemotherapy in locally advanced rectal cancer. J Nucl Med 2006;47:1241-1248.

[10] Rosenberg R, Herrmann K, Gertler R, et al. The predictive value of metabolic response to preoperative radiochemotherapy in locally advanced rectal cancer measured by PET/CT. Int J Colorectal Dis 2009;24:191-200.

[11] Hindie E, Hennequin C, Moretti JL. Predicting response to chemoradiotherapy in rectal and oesophageal cancer with 18F-FDG: prognostic value and possible role in patient management. Eur J Nucl Med Mol Imaging 2007;34:1576-1582.

[12] Janssen MH, Ollers MC, Riedl RG, et al. Accurate Prediction of Pathological Rectal Tumor Response after Two Weeks of Preoperative Radiochemotherapy Using (18)F-FluorodeoxyglucosePositron Emission Tomography-Computed Tomography Imaging. Int J Radiat Oncol Biol Phys 2010; 77: 392-399.

[13] Young $\mathrm{H}$, Baum R, Cremerius U, et al. Measurement of clinical and subclinical tumour response using [18F]-fluorodeoxyglucose and positron emission tomography: review and 1999 EORTC recommendations. European Organization for Research and Treatment of Cancer (EORTC) PET Study Group. Eur J Cancer 1999;35:1773-1782.

[14] de Geus-Oei LF, Vriens D, van Laarhoven HW, et al. Monitoring and predicting response to therapy with 18F-FDG PET in colorectal cancer: a systematic review. J Nucl Med 2009;50 Suppl 1:43S-54S.

[15] Beaulieu S, Kinahan P, Tseng J, et al. SUV varies with time after injection in (18)F-FDG PET of breast cancer: characterization and method to adjust for time differences. J Nucl Med 2003;44:1044-1050.

[16] Ollers M, Bosmans G, van Baardwijk A, et al. The integration of PET-CT scans from different hospitals into radiotherapy treatment planning. Radiother Oncol 2008;87:142-146.

[17] Daisne JF, Sibomana M, Bol A, et al. Tri-dimensional automatic segmentation of PET volumes based on measured source-to-background ratios: influence of reconstruction algorithms. Radiother Oncol 2003;69:247-250. 
[18] Mandard AM, Dalibard F, Mandard JC, et al. Pathologic assessment of tumor regression after preoperative chemoradiotherapy of esophageal carcinoma. Clinicopathologic correlations. Cancer 1994;73:2680-2686.

[19] Rodel C, Martus P, Papadoupolos T, et al. Prognostic significance of tumor regression after preoperative chemoradiotherapy for rectal cancer. J Clin Oncol 2005;23:8688-8696.

[20] Vecchio FM, Valentini V, Minsky BD, et al. The relationship of pathologic tumor regression grade (TRG) and outcomes after preoperative therapy in rectal cancer. Int J Radiat Oncol Biol Phys 2005;62:752-760.

[21] Veenhof AA, Bloemena E, Engel AF, et al. The relationship of histological tumor regression grade (TRG) and two different time intervals to surgery following radiation therapy for locally advanced rectal cancer. Int J Colorectal Dis 2009;24:1091-1096.

[22] Habr-Gama A, Perez RO, Proscurshim I, et al. Interval between surgery and neoadjuvant chemoradiation therapy for distal rectal cancer: does delayed surgery have an impact on outcome? Int J Radiat Oncol Biol Phys 2008;71:1181-1188.

[23] Dolinsky CM, Mahmoud NN, Mick R, et al. Effect of time interval between surgery and preoperative chemoradiotherapy with 5-fluorouracil or 5-fluorouracil and oxaliplatin on outcomes in rectal cancer. J Surg Oncol 2007;96:207-212.

[24] Kao PF, Chou YH, Lai CW. Diffuse FDG uptake in acute prostatitis. Clin Nucl Med 2008;33:308-310.

[25] Keyes JW, Jr. SUV: standard uptake or silly useless value? J Nucl Med 1995;36:1836-1839.

[26] Hamberg LM, Hunter GJ, Alpert NM, et al. The dose uptake ratio as an index of glucose metabolism: useful parameter or oversimplification? J Nucl Med 1994;35:1308-1312.

[27] Boellaard R, O'Doherty MJ, Weber WA, et al. FDG PET and PET/CT: EANM procedure guidelines for tumour PET imaging: version 1.0. Eur J Nucl Med Mol Imaging 2009;37:181-200.

[28] Janssen $M H$, Ollers MC, Stiphout RG, et al. Blood glucose level normalization and accurate timing improves the accuracy of PET-based treatment response predictions in rectal cancer. Radiother Oncol. 2010;95: 203-208 


\title{
Part II:
}

\author{
Perfusion-CT imaging during \\ short course hypofractionated \\ radiotherapy.
}





\section{Chapter}

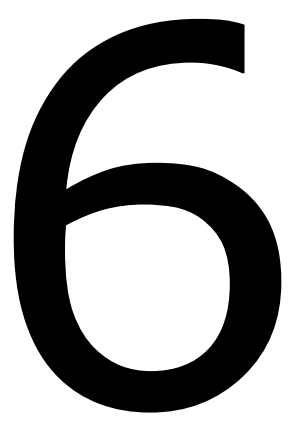

\section{Tumor perfusion increases during hypofractionated short course radiotherapy in rectal cancer: sequential perfusion-CT findings.}

Marco Janssen

Hugo Aerts

Roel Kierkels

Walter Backes

Michel Öllers

Jeroen Buijsen

Philippe Lambin

Guido Lammering

Radiotherapy \& Oncology, 2010; 94(2): 156-160 


\section{Abstract \\ Purpose}

The purpose of this study was to investigate perfusion of rectal tumors and to determine early responses to short course hypofractionated radiotherapy (RT).

\section{Materials \& Methods}

Twenty-three rectal cancer patients were included, which underwent perfusion-CT (pCT) imaging before (pre-scan) and after treatment (post-scan). Contrastenhancement was measured in tumor- and muscle tissue and in the external iliacartery. Perfusion was quantified with three pharmacokinetic parameters: $K^{\text {trans }}, v_{e}$ and $v_{p}$. Perfusion differences between tumor and normal tissue and changes of the pharmacokinetic-parameters between both scans were evaluated.

\section{Results}

The median tumors $K^{\text {trans }}$ values increased significantly from the pre-scan $(0.36 \pm 0.11$ $\left.\left[\mathrm{min}^{-1}\right]\right)$ to the post-scan $\left(0.44 \pm 0.13\left[\mathrm{~min}^{-1}\right]\right)(\mathrm{p}<0.001)$. Also, histogram analysis showed a shift of tumor voxels from lower $K^{\text {trans }}$ values towards higher $K^{\text {trans }}$ values. Furthermore, the median $K^{\text {trans }}$ values were significantly higher for tumor than for muscle tissue on both the pre-scan $\left(0.10 \pm 0.05\left[\mathrm{~min}^{-1}\right], p<0.001\right)$ and the post-scan $(0.10 \pm 0.04$ $\left.\left[\mathrm{min}^{-1}\right], \mathrm{p}<0.001\right)$. In contrast, no differences between tumor and muscle tissue were found for $v_{e}$ and $v_{p}$. Also, no significant differences were observed for $v_{e}$ and $v_{p}$ between the two pCT-imaging time-points.

\section{Conclusions}

Hypofractionated radiotherapy of rectal cancer leads to an increased tumor perfusion as reflected by an elevated $K^{\text {trans }}$, possibly improving the bioavailability of cytotoxic agents in rectal tumors, often administered early after radiotherapy treatment. 


\subsection{Introduction}

Radiotherapy (RT), alone or with chemotherapy, is an established treatment for patients diagnosed with rectal cancer ${ }^{1-3}$. In tumors judged to be resectable, preoperative RT is primarily used to lower the risk of local failure ${ }^{4-6}$. For this purpose, short course hypofractionated RT ( $5 \times 5$ Gy) followed by immediate surgery has been extensively used. However, several trial initiatives are currently ongoing to modulate the schedule of short course hypofractionated RT from immediate surgery to a planned delay before surgery with the possible advantage of tumor down-sizing. Further knowledge of the biological changes of the tumor during short course RT would be useful to optimize the treatment management and to improve the development of response predictors, allowing individualized treatment.

Perfusion Computed-Tomography ( $\mathrm{pCT}$ ) imaging is increasingly used in clinical studies as a non-invasive technique to assess the microvascular status of tumor tissue ${ }^{7-13}$. pCT-imaging is a dynamic imaging technique, which can give insight in the uptake kinetics of the administered tracer by pharmacokinetic modeling ${ }^{14}$. A for PCT-imaging commonly applied pharmacokinetic two-compartment model for perfusion imaging is the extended Kety-model, with the following pharmacokinetic parameters: the transendothelial volume transfer constant $K^{\text {trans }}$, the fractional volume of the extravascularextracellular space (EES) $\left(v_{e}\right)$ and the fractional blood plasma volume $v_{p}{ }^{14,15}$. For cancer research, $K^{\text {trans }}$, describing the transfer rate of the contrast agent from the blood plasma into the EES, is the most valuable pharmacokinetic parameter, related to the microvascular blood flow, vessel wall permeability and vessel density ${ }^{14}$.

pCT-measurements have been shown to serve as early markers of treatment response ${ }^{9-12,16}$. Tumors with a high $K^{\text {trans }}$ tend to better respond to chemotherapy and/or radiotherapy treatment than tumors with lower values of $K^{\text {trans }}$, blood volume and/or blood flow ${ }^{9-12,16-18}$. However, little is known about therapy-related changes of the perfusion parameters of tumor tissue. Wang et al. presented the predictive strength of repeated PCT-imaging, with a decrease of the permeability being predictive for a higher progression-free survival period in non-small cell lung cancer patients treated with chemotherapy ${ }^{12}$. To the best of our knowledge, no study has yet examined early changes in tumor perfusion in response to radiotherapy treatment of rectal cancer.

The purpose of this study was to investigate perfusion of rectal tumors and responses to hypofractionated short course RT. This could give important insight into early changes in the tumor microcirculation during radiotherapy and might help to better predict tumor response. 


\subsection{Materials \& Methods \\ Patient Characteristics}

Twenty-three patients, diagnosed with non-locally advanced rectal cancer (NLARC), were included in this study. Based on pre-treatment magnetic resonance imaging (MRI), the clinical TNM staging was staged as T I-III, N 0-II, M 0-I. All patients were referred to pre-operative treatment with short course RT, 5 fractions of 5 Gy on 5 consecutive working days, followed by a total mesorectal excision (TME) within 3 days after the last RT fraction. According to the Dutch law, the medical ethics committee approved the trial and all patients gave written informed consent before entering the study.

\section{PET-CT and $\mathrm{PCT}$ acquisition}

All patients underwent FDG-PET-CT and pCT imaging at two time points: prior to the start of therapy and at the day of the last RT fraction. All PET-CT and PCT examinations were performed on the same dedicated Siemens TruePoint Biograph 40 PET-CT simulator (Siemens Medical, Erlangen, Germany). The patients were positioned equal to the radiotherapy treatment position using a laser alignment system to have minimal variations between imaging and treatment conditions and between the two imaging time-points. For the PET-CT scan, an intravenous injection of FDG (weight [kg] $* 4+20$ $\mathrm{MBq}$ ) was performed. For PET reconstruction (OSEM2D: 4 iterations, 8 subsets), CT based attenuation correction; 3D scatter- and decay-correction were performed. After the PET-CT scan, a PCT-scan was performed over 100 seconds. The volume of interest (VOI) for the pCT-scan was defined by an expert radiation oncologist (J.B. or G.L.) with knowledge of the PET-data. To ensure that the most representative tumor area was chosen, the tumor area with the highest FDG-uptake on the PET-scan was selected. Knowledge of the FOV selected for the first PCT-scan was used to select the identical region for the second PCT-scan. For the PCT-scan, a volume of 120 of an iodinated contrast-agent (300 mg iodine/ml, Xenetix 300, Guerbet, Aulnay-sous-Bois, France) was injected at a rate of $3 \mathrm{ml} / \mathrm{s}$ via an automatic injector (Stellant Sx, CT Injection System, MedRad, Warrendale, USA) into the antecubital fossa. The pCT-scan was performed in a static cine-mode over 12 contiguous slices with a slice thickness of 2.4 $\mathrm{mm}$, a field-of-view of $500 \mathrm{~mm}$ and an image size of 512x512 pixels. Other acquisition settings were: tube voltage $80 \mathrm{kVp}$, tube current $140 \mathrm{mAs}$ and a rotation time of 1 second.

\section{Pharmacokinetic Analysis}

Automatic image-registration between the static PET-CT scan and the pCT-scan was performed based on mutual-information (Focal software, version 4.34, CMS Inc., St. Louis, Missouri). For each PET-CT scan, the tumor was delineated with dedicated software (TrueD VC50, Siemens MI, Erlangen, Germany) using automated SUVthresholding of the PET-images with the threshold (percentage of SUV $\mathrm{Vax}_{\max }$ within the tumor) depending on the tumor-to-background signal ratio, with the gluteus muscle selected as relevant background tissue ${ }^{19,20}$. As a reference sample for the pharmacokinetic analysis, an additional VOI was manually selected within the gluteus muscle to check for possible changes of the pharmacokinetic parameters of muscle tissue outside 
the irradiated volume. When quantifying the pharmacokinetic parameters of muscle tissue, a VOI was manually drawn within both the left and right gluteus muscle. The median values of the pharmacokinetic parameters within both VOIs were averaged to account for intra-tissue heterogeneity of the muscle tissue. The resulting contours of both tumor- and muscle tissue were projected on the registered $\mathrm{pCT}$. The $\mathrm{pCT}$ data were down-sampled from a voxel size of $0.98 \times 0.98 \times 2.4 \mathrm{~mm}$ to $3.92 \times 3.92 \times 4.8 \mathrm{~mm}$ to improve the signal-to-noise ratio (SNR). For the quantification of the dynamic pCTdata, the extended Kety-model was used, describing the uptake of a contrast agent from the blood plasma into the tissue by ${ }^{14}$ :

$$
C_{t}(t)=v_{p} C_{p}(t)+K^{\text {trans }} \int_{0}^{t} C_{p}(u) e^{\frac{-K^{\text {trans }}}{v_{e}}(t-u)} d u
$$

The blood plasma concentration curve $\left(C_{p}\right)$, extracted from the right external iliac artery, was derived from the acquired whole blood tracer concentration $\left(C_{b}\right)$ divided by (1-Hct), with the hematocrit value (Hct) set to 0.45 (Fig. 6.2) ${ }^{14}$. To improve the SNR, $C_{p}$ was calculated by averaging the concentration time curves over all voxels selected inside the iliac artery. The tumor and muscle tissue concentration curves $\left(C_{t}\right)$ were extracted from the dynamic pCT data on a voxel-by-voxel basis and on a tumor uptake curve based on the average of all tumor voxels (Fig. 6.2). Pharmacokinetic analysis was performed using in-house developed software in MATLAB (R2008b, The Mathworks Inc., Natick, USA). The concentration time curves from pCT-data were fitted to the pharmacokinetic model, with the pharmacokinetic parameters being calculated using the Levenberg-Marquardt algorithm, with boundaries set to $0 \leq K^{\text {trans }} \leq 5 \min ^{-1}, 0 \leq v_{e} \leq 1$ and $0 \leq v_{p} \leq 1{ }^{21-23}$.

\section{Statistical Analysis}

All data are expressed as means \pm standard deviation (SD) and range (min. to max.). Statistical differences between the parameters were evaluated in SPSS (version 15.0, SPSS Inc., Chicago, IL, USA), performing a Wilcoxon-signed-rank test for the comparison of related measurements. Differences were considered to be significant when the p-value was less than 0.05 .

\subsection{Results}

\section{Perfusion of tumor and muscle tissue}

To study differences in the perfusion of tumor and normal tissue, 23 patients diagnosed with rectal cancer underwent sequential pCT-imaging before and after treatment. From these scans, the pharmacokinetic parameters of tumor and normal tissue were calculated at both imaging time points. In figure 6.1, CT-images of a representative patient are shown with the perfusion $K^{\text {trans }}$ maps of both tumor and muscle tissue at both time-points. Visual interpretation of the $K^{\text {trans }}$ maps showed a higher and more heterogeneous uptake in tumor tissue compared to muscle tissue on both scans. Note the increased $K^{\text {trans }}$ in tumor tissue on the post-treatment scan compared to the pretreatment scan, whereas $K^{\text {trans }}$ in muscle tissue presented similar patterns at both imaging time-points. 


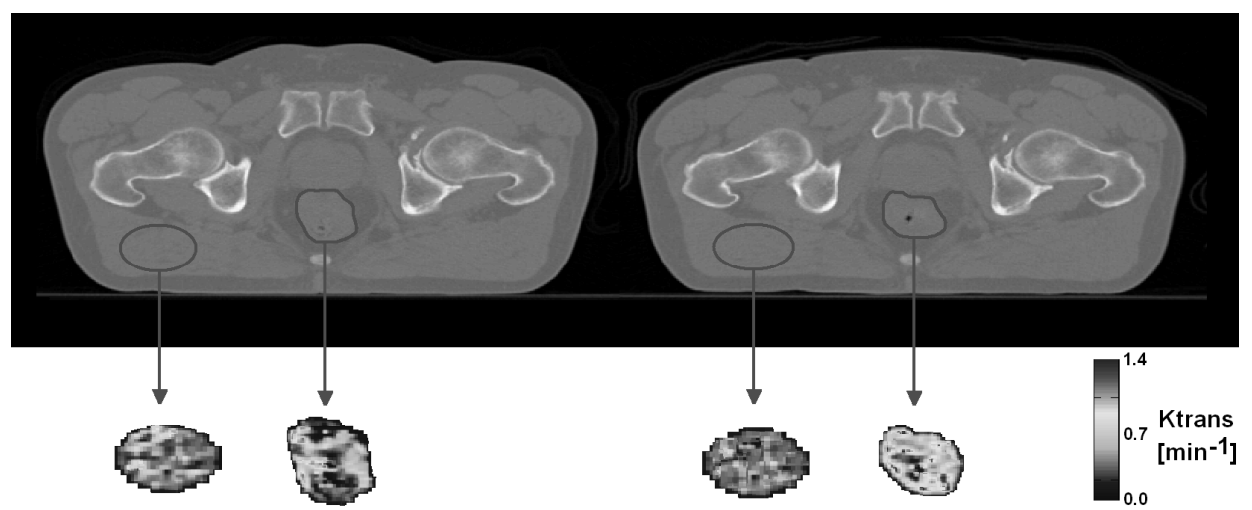

Figure 6.1: Comparison of a pre-treatment (left) and post-treatment (right) perfusion-CT scan for a representative patient. The upper row displays the anatomic pre-contrast CT-images, the lower row the $K^{\text {trans }}$ maps of the regions of interest selected in muscle and tumor tissue. Note the increased values of $K^{\text {trans }}$ in the tumor tissue on the post-treatment scan compared to the pre-treatment scan, whereas muscle tissue presents with similar patterns of $K^{\text {trans }}$ at both imaging time-points.

\section{Perfusion parameters before and after short course radiotherapy}

For the quantification of the pharmacokinetic parameters, the median $K^{\text {trans }}, v_{e}$ and $v_{p}$ values of tumor and muscle tissue were calculated at both time-points. Figure 6.2 shows scatterplots of the median parameter values for tumor and muscle tissue. After $\mathrm{RT}$, an increase of the median $K^{\text {trans }}$ within the tumor was found for all patient except one, resulting in an average increase of $K^{\text {trans }}$ of $25.4 \pm 28.7 \%$ (range: -3.8 to $113.1 \%)(p<0.001)$. However, the median $K^{\text {trans }}$ values within the muscle tissue was not significantly different between the two imaging time points $(p=0.554)$. The other two pharmacokinetic parameters, $v_{e}$ and $v_{p}$, showed no significantly different values between the two time-points and between tumor and muscle tissue. In Table 6.1, an overview of all median parameter values are shown with the corresponding statistics. In figure 6.3, the average histogram of the $K^{\text {trans }}$ values within the tumor are shown for all patients. As can be seen from the histogram, pre-operative treatment with short course hypofractionated RT resulted in an increased tumor perfusion for the included patients. For the bins of the histogram presenting the number of voxels with a relatively high $K^{\text {trans }}$ value, an increase of the number of voxels was observed between the preand post-treatment PCT-scan. In contradiction, a decrease was found for the number of voxels in the bins with a lower $K^{\text {trans }}$ value. The shift of the histogram towards increased bins with relatively higher $K^{\text {trans }}$ indicates an increase of tumor perfusion due to pre-operative treatment with short course hypofractionated RT. 
Tumor Tissue
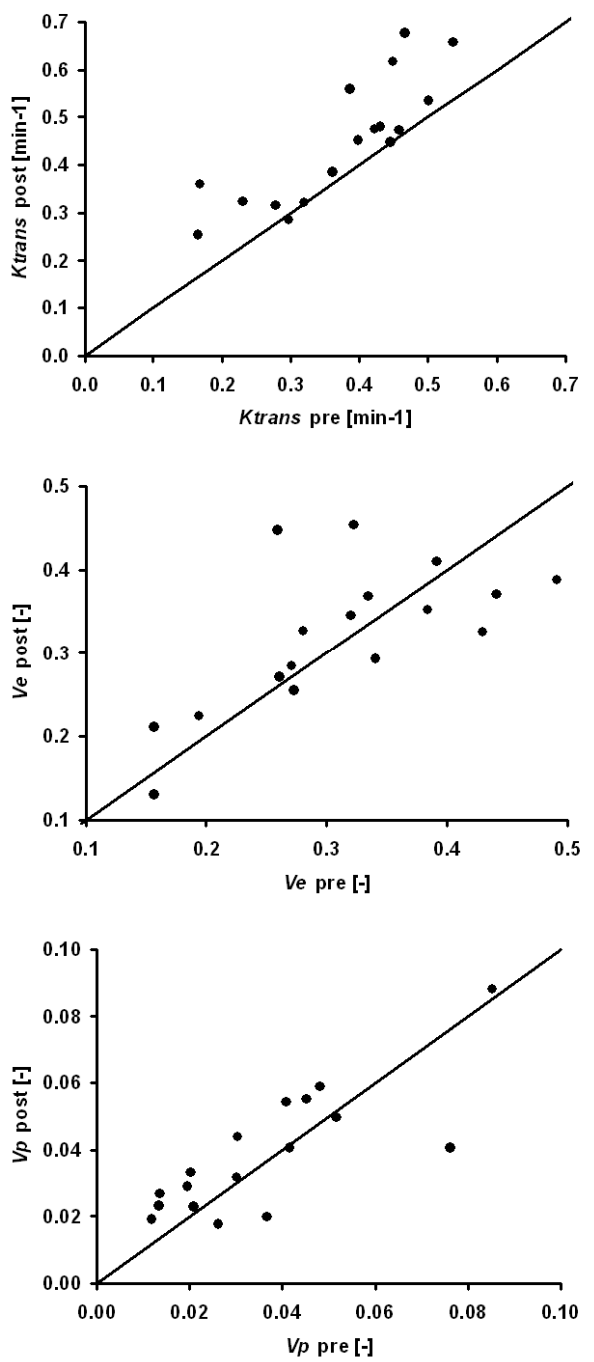

Muscle Tissue
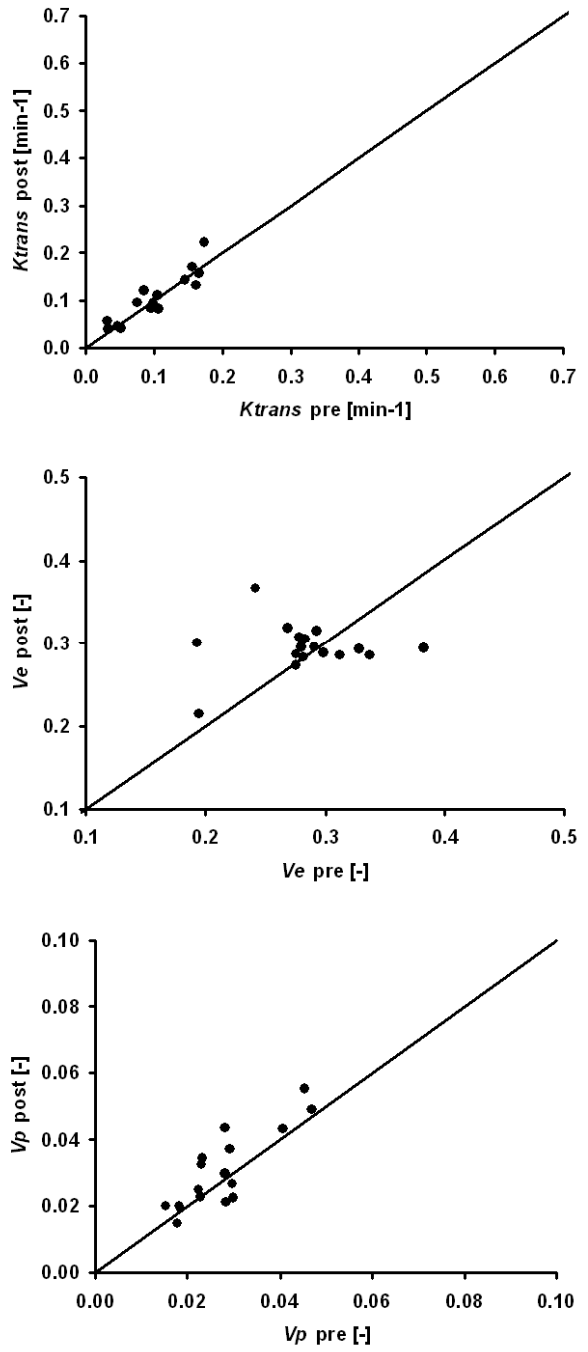

Figure 6.2: Scatterplots showing the median $K^{\text {trans }}, v_{e}$ and $v_{p}$ values within tumor- and muscle tissue at the perfusion-CT-scans acquired before (pre) and after (post) pre-operative treatment with hypofractionated radiotherapy. Each dot represents the median value of the pharmacokinetic parameter of one patient. Note the increase of $K^{\text {trans }}$ within the tumor after treatment with hypofractionated radiotherapy, whereas the median $K^{\text {trans }}$ values of muscle tissue outside of the irradiated volume remained stable. 


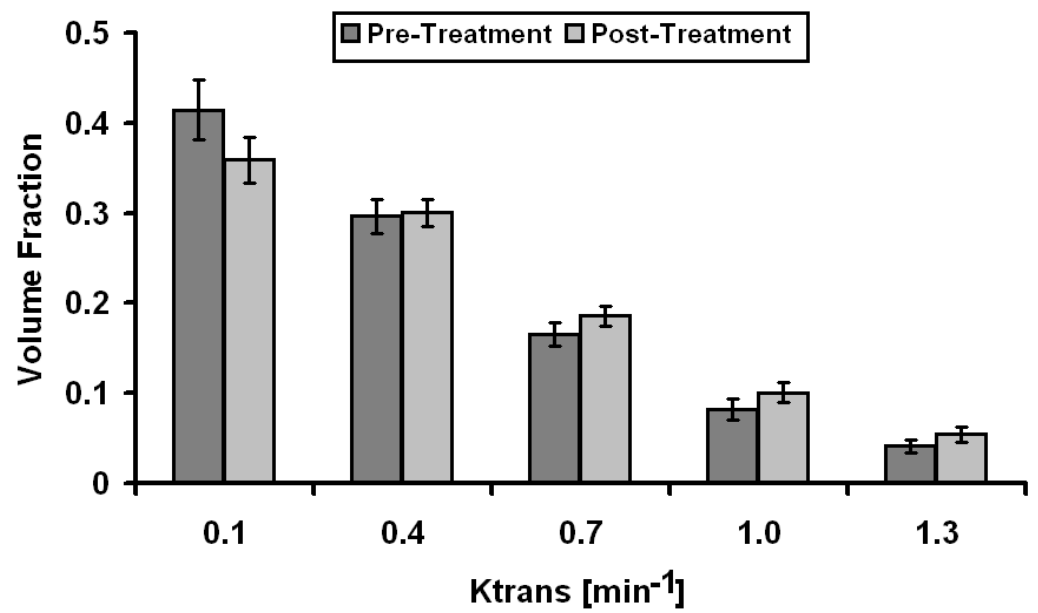

Figure 6.3: Average histograms of the $K^{\text {trans }}$ values within the tumor for the pre-treatment (dark boxes) and post-treatment scan (light boxes). The bars represent the mean and the error-bars the standard deviation of the $K^{\text {trans }}$ estimates within the bin. Note the increase of the number of voxels within the bins with a higher $K^{\text {trans }}$ value $(>0.4)$ after pre-operative treatment with short course hypofractionated radiotherapy, indicating an increase of tumor perfusion after treatment with hypofractionated radiotherapy.

Table 6.1: Overview of the median pharmacokinetic parameters, $K^{\text {trans }}, v_{e}$ and $v_{p}$, in tumor- and muscle tissue both pre- and post-treatment.

\begin{tabular}{|c|c|c|c|c|}
\hline & \\
\hline & & pre-RT & post-RT & p-value \\
\hline$\kappa^{\text {trans }}\left[\mathrm{min}^{-1}\right]$ & tumor tissue & $0.36 \pm 0.11$ & $0.44 \pm 0.13$ & $<0.001$ \\
\hline & muscle tissue & $0.10 \pm 0.05$ & $0.10 \pm 0.05$ & 0.554 \\
\hline & p-value & $<0.001$ & $<0.001$ & \\
\hline
\end{tabular}

\begin{tabular}{|c|c|c|c|c|}
\hline$v_{e}[-]$ & tumor tissue & $0.31 \pm 0.10$ & $0.32 \pm 0.09$ & 0.795 \\
\hline & muscle tissue & $0.28 \pm 0.05$ & $0.29 \pm 0.03$ & 0.381 \\
\hline & p-value & 0.356 & 0.344 & \\
\hline
\end{tabular}

\begin{tabular}{|c|c|c|c|c|}
\hline$v_{p}[-]$ & tumor tissue & $0.04 \pm 0.02$ & $0.04 \pm 0.02$ & 0.124 \\
\hline & muscle tissue & $0.03 \pm 0.01$ & $0.03 \pm 0.01$ & 0.098 \\
\hline & p-value & 0.266 & 0.266 & \\
\hline
\end{tabular}




\subsection{Discussion}

The purpose of this study was to characterize changes of tumor perfusion after short course hypofractionated radiotherapy treatment. The pharmacokinetic parameters of rectal tumors revealed significant higher $K^{\text {trans }}$ values compared to muscle tissue at both the pre- and post-treatment PCT-scan. Short course RT resulted in a significant increase of the median $K^{\text {trans }}$ in tumor tissue, indicating an early increase in tumor perfusion already on the last day of pre-operative RT. The significant increase of tumor perfusion during the rather short time interval between the pre- and post-treatment PCT-scan could be explained by first endothelial cell death within the irradiated volumes, resulting in endothelial cell leakage which in turn results in increased values of $K^{\text {trans }}$ within the tumor. The VOI manually selected within muscle tissue was chosen outside of the irradiated volume, so no endothelial cell death was expected to occur within the VOls of muscle tissue. An increase in tumor perfusion early during preoperative treatment might improve the availability of cytotoxic agents of chemotherapy to the tumor, often administered after finishing the radiotherapy treatment. The data as presented within this study could serve as reference-data of perfusion changes within the tumor during RT-treatment for future studies combining a short course hypofractionated radiotherapy course with anti-angiogenic agents (e.g. rapamycin).

In general, perfusion measurements are intrinsically variable due to internal and external factors, including day-to-day physiological variations, technical variability, observer variability and intra-tissue heterogeneity ${ }^{24}$. The used acquisition time of 100 seconds was long enough to perform measurements of $K^{\text {trans }}$, whereas measurements of $v_{e}$ require imaging times which incorporate the maximal signal enhancement in the tumor tissue, about 120 to 180 seconds after injection of the contrast agent ${ }^{25,26}$.

For this reason, the relatively short imaging time used within this study might bias the $v_{e}$ values measured within this study. The used sampling time of 1 second was expected to be sufficient for reliable estimations of $K^{\text {trans }}$ and $v_{p}{ }^{15,26}$.

One of the major limitations of this study was the relatively small dimension of the FOV of the PCT-scan in craniocaudal direction. The craniocaudal coverage of the pCT FOV of only $2.88 \mathrm{~cm}$ resulted for some patients in an incomplete coverage of the rectal tumor. For these patients, PCT measurements were obtained from the tumor region showing the highest FDG-uptake, as assessed from the static PET-CT scan. Due to incomplete tumor coverage, the calculated median values of the three investigated pharmacokinetic parameters only represent the perfusion of the tumor region covered by the FOV. However, Goh et al. presented a study showing that an increased FOV in craniocaudal direction does not improve the reproducibility of perfusion measurements ${ }^{27}$. Another limitation was that day-to-day differences in bladder and rectum filling, hampering a voxel-wise comparison of the pharmacokinetic parameter maps of the pre- and post-treatment PCT-scans within this study. Due to large differences in bladder or rectum filling for some of the included patients, a rigid-registration was not sufficient to enable a correct registration of the pre- and post-treatment PCT-scans. However, available non-rigid registration algorithms are not yet properly validated for rectal cancer. 
In conclusion, perfusion-CT measurements of rectal tumors enable the assessment of changes of tumor perfusion resulting from radiotherapy treatment. Short course hypofractionated radiotherapy of rectal cancer significantly increased tumor perfusion $\left(K^{\text {trans }}\right)$, which might improve the bioavailability of cytotoxic agents in rectal tumor, often administered to the patient after radiotherapy treatment. 


\subsection{References}

[1] Frykholm, G, Glimelius, B, Pahlman, L. Preoperative irradiation with and without chemotherapy (MFL) in the treatment of primarily non-resectable adenocarcinoma of the rectum. Results from two consecutive studies. Eur J Cancer Clin Oncol 1989;25:1535-1541.

[2] Sauer, R, Becker, H, Hohenberger, W, et al. Preoperative versus postoperative chemoradiotherapy for rectal cancer. N Engl J Med 2004;351:1731-1740.

[3] Sebag-Montefiore, D. Developments in the use of chemoradiotherapy in rectal cancer. Colorectal Dis 2006;8 Suppl 3:14-17.

[4] Improved survival with preoperative radiotherapy in resectable rectal cancer. Swedish Rectal Cancer Trial. N Engl J Med 1997;336:980-987.

[5] Kapiteijn, E, Marijnen, CA, Nagtegaal, ID, et al. Preoperative radiotherapy combined with total mesorectal excision for resectable rectal cancer. N Engl J Med 2001;345:638-646.

[6] Peeters, KC, Marijnen, CA, Nagtegaal, ID, et al. The TME trial after a median follow-up of 6 years: increased local control but no survival benefit in irradiated patients with resectable rectal carcinoma. Ann Surg 2007;246:693-701.

[7] Miles, KA. Perfusion CT for the assessment of tumour vascularity: which protocol? $\mathrm{Br} \mathrm{J}$ Radiol 2003;76 Spec No 1:S36-42.

[8] Goh, V, Padhani, AR, Rasheed, S. Functional imaging of colorectal cancer angiogenesis. Lancet Oncol 2007;8:245-255.

[9] Sahani, DV, Kalva, SP, Hamberg, LM, et al. Assessing tumor perfusion and treatment response in rectal cancer with multisection CT: initial observations. Radiology 2005;234:785-792.

[10] Bellomi, M, Petralia, G, Sonzogni, A, Zampino, MG, Rocca, A. CT perfusion for the monitoring of neoadjuvant chemotherapy and radiation therapy in rectal carcinoma: initial experience. Radiology 2007;244:486-493.

[11] Park, MS, Klotz, E, Kim, MJ, et al. Perfusion CT: noninvasive surrogate marker for stratification of pancreatic cancer response to concurrent chemo- and radiation therapy. Radiology 2009;250:110-117.

[12] Wang, J, Wu, N, Cham, MD, Song, Y. Tumor response in patients with advanced non-small cell lung cancer: perfusion CT evaluation of chemotherapy and radiation therapy. AJR Am J Roentgenol 2009;193:1090-1096.

[13] Harvey, C, Dooher, A, Morgan, J, Blomley, M, Dawson, P. Imaging of tumour therapy responses by dynamic CT. Eur J Radiol 1999;30:221-226.

[14] Tofts, PS, Brix, G, Buckley, DL, et al. Estimating kinetic parameters from dynamic contrastenhanced T(1)-weighted MRI of a diffusable tracer: standardized quantities and symbols. J Magn Reson Imaging 1999;10:223-232.

[15] Aerts, HJ, van Riel, NA, Backes, WH. System identification theory in pharmacokinetic modeling of dynamic contrast-enhanced MRI: influence of contrast injection. Magn Reson Med 2008;59:11111119.

[16] Zima, A, Carlos, R, Gandhi, D, Case, I, Teknos, T, Mukherji, SK. Can pretreatment CT perfusion predict response of advanced squamous cell carcinoma of the upper aerodigestive tract treated with induction chemotherapy? AJNR Am J Neuroradiol 2007;28:328-334.

[17] Hermans, R, Lambin, P, Van der Goten, A, et al. Tumoural perfusion as measured by dynamic computed tomography in head and neck carcinoma. Radiother Oncol 1999;53:105-111.

[18] Hermans, R, Van den Bogaert, W. Outcome prediction after surgery and chemoradiation of headand-neck squamous cell carcinoma (HNSCC), using baseline perfusion computed tomography (CT) microcirculatory parameters vs. tumor volume. Int J Radiat Oncol Biol Phys 2009;74:1307; author reply 1307.

[19] Daisne, JF, Sibomana, M, Bol, A, Doumont, T, Lonneux, M, Gregoire, V. Tri-dimensional automatic segmentation of PET volumes based on measured source-to-background ratios: influence of reconstruction algorithms. Radiother Oncol 2003;69:247-250.

[20] Ollers, M, Bosmans, G, van Baardwijk, A, et al. The integration of PET-CT scans from different hospitals into radiotherapy treatment planning. Radiother Oncol 2008;87:142-146.

[21] Harrer, JU, Parker, GJ, Haroon, HA, et al. Comparative study of methods for determining vascular permeability and blood volume in human gliomas. J Magn Reson Imaging 2004;20:748-757. 
[22] Jackson, A, Jayson, GC, Li, KL, et al. Reproducibility of quantitative dynamic contrast-enhanced MRI in newly presenting glioma. Br J Radiol 2003;76:153-162.

[23] Walker-Samuel, S, Leach, MO, Collins, DJ. Reference tissue quantification of DCE-MRI data without a contrast agent calibration. Phys Med Biol 2007;52:589-601.

[24] Goh, V, Halligan, S, Hugill, JA, Gartner, L, Bartram, Cl. Quantitative colorectal cancer perfusion measurement using dynamic contrast-enhanced multidetector-row computed tomography: effect of acquisition time and implications for protocols. J Comput Assist Tomogr 2005;29:59-63.

[25] Henderson, E, Rutt, BK, Lee, TY. Temporal sampling requirements for the tracer kinetics modeling of breast disease. Magn Reson Imaging 1998;16:1057-1073.

[26] Kierkels, RG, Backes, WH, Janssen, MH, et al. Comparison Between Perfusion Computed Tomography and Dynamic Contrast-Enhanced Magnetic Resonance Imaging in Rectal Cancer. Int J Radiat Oncol Biol Phys 2010; 77: 400-408.

[27] Goh, V, Halligan, S, Gartner, L, Bassett, P, Bartram, Cl. Quantitative colorectal cancer perfusion measurement by multidetector-row $\mathrm{CT}$ : does greater tumour coverage improve measurement reproducibility? Br J Radiol 2006;79:578-583. 


\section{Chapter}

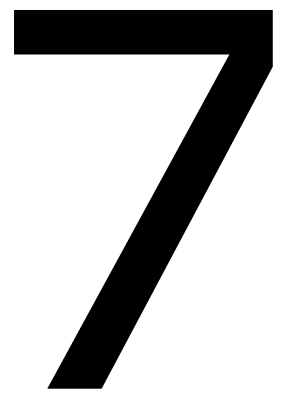

\section{Repeated PET-CT and perfusion-CT imaging in rectal cancer: FDG uptake corresponds with tumor perfusion.}

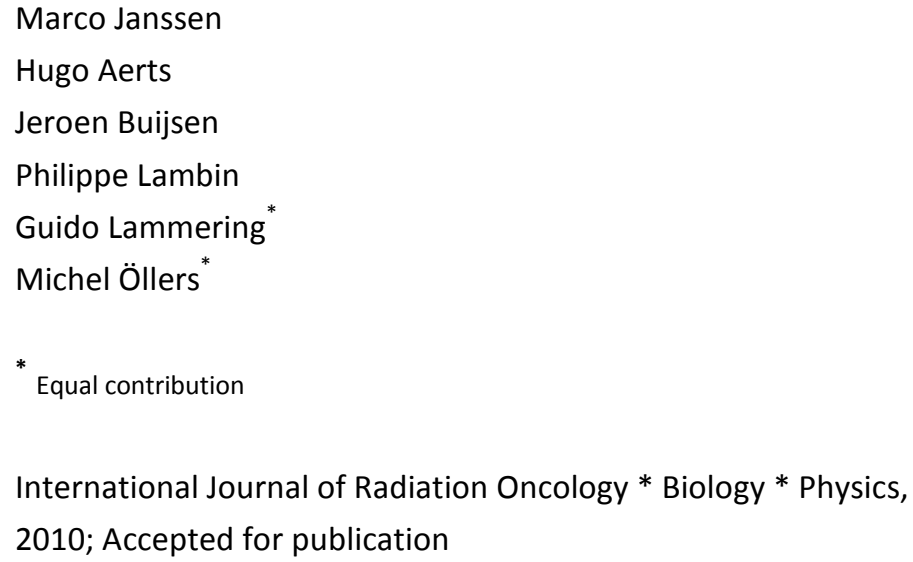




\section{Abstract \\ Purpose}

The purpose of this study was to analyze the intratumoral FDG uptake and perfusion within rectal tumors prior to and after hypofractionated radiotherapy.

\section{Materials \& Methods}

Rectal cancer patients, referred for preoperative hypofractionated radiotherapy (RT), underwent FDG-PET-CT and perfusion-CT ( $\mathrm{pCT}$ ) imaging prior to the start of hypofractionated RT and at the day of the last RT-fraction. The pCT-images were analyzed using the extended Kety-model, quantifying tumor perfusion with the pharmacokinetic parameters $K^{\text {trans }}, v_{e}$ and $v_{p}$. The mean and maximum FDG-uptake (SUV) and transfer constant $\left(K^{\text {trans }}\right)$ within the tumor were correlated. Also, the tumor was subdivided into eight sub-regions and for each region, the mean and maximum SUVs and $K^{\text {trans }}$ values were assessed and correlated. Furthermore, the mean FDG-uptake in voxels presenting with the lowest $25 \%$ of perfusion was compared to the FDG uptake in the voxels with the $25 \%$ highest perfusion.

\section{Results}

The mean and maximum $K^{\text {trans }}$ values were positively correlated with the corresponding SUVs $(\rho=0.596, p=0.001$ and $\rho=0.779, p<0.001)$. Also, positive correlations were found for $K^{\text {trans }}$ values and SUVs within the sub-regions (mean: $\rho=0.413, p<0.001$ and max: $\rho=0.540, p<0.001$ ). The mean FDG uptake in the $25 \%$ highest perfused tumor regions was significantly higher compared to the $25 \%$ lowest perfused regions $(10.6 \pm 5.1 \%, p=0.017)$. During hypofractionated radiotherapy, stable mean $(p=0.379)$ and maximum ( $p=0.280)$ FDG uptake levels were found, whereas the mean $(p=0.040)$ and maximum ( $p=0.003) K^{\text {trans }}$ values were found to significantly increase.

\section{Conclusions}

Highly perfused rectal tumors presented with higher FDG-uptake levels compared to relatively low perfused tumors. Also, intra-tumor regions with a high FDG uptake demonstrated with higher levels of perfusion than regions with a relatively low FDGuptake. Early after hypofractionated RT, stable FDG uptake levels were found, whereas tumor perfusion was found to significantly increase. 


\subsection{Introduction}

Radiotherapy (RT), alone or with chemotherapy, is an established treatment for patients diagnosed with rectal cancer ${ }^{1-3}$. The use of sequential fluorodeoxyglucose positron-emission-tomography (FDG-PET) and perfusion-CT (pCT) imaging allows a closer look at therapy related changes of the tumor's metabolic activity and tumor perfusion, which might enable tumor response predictions and tailored therapies in the future ${ }^{4-7}$. Evaluation of the metabolic activity of malignancies, assessed with sequential FDG-PET-imaging, has been proven to be an accurate and promising method for the prediction of treatment response during and after neo-adjuvant chemoradiotherapy (CRT) in an increasing number of malignancies ${ }^{5,8-19}$. The response index, describing the percentage of reduction of SUV $V_{\max }$ after two weeks of pre-operative CRT treatment has been presented as an accurate predictor of the pathological treatment response ${ }^{5,16-19}$. Also, tumors with higher FDG uptake level prior to the start of treatment presented with a higher decrease of the standardized uptake values (SUVS) early during treatment compared to tumors with a lower pre-treatment FDG uptake ${ }^{18,19}$. Therefore, an accurate prediction of the pathological tumor response already early during pre-operative treatment would enable more individualized treatment regimens, aiming at further improvement of the tumor response or a modified surgical approach.

Except changes in the metabolic activity of the tumor, also assessments of the microvascular status of the tumor tissue using $\mathrm{PCT}$ measurements have been presented as an early marker of treatment response ${ }^{20-24}$. PCT imaging is increasingly applied to non-invasively assess the microvascular status of tumor tissue by studying the uptake kinetics of the administered tracer over time ${ }^{7,20-23,25-34}$. A two-compartment model (extended Kety model) is commonly used for pharmacokinetic modeling of the tracer's uptake kinetics, quantifying tumor perfusion with the pharmacokinetic parameters $K^{\text {trans }}, \mathrm{v}_{\mathrm{e}}$ and $\mathrm{v}_{\mathrm{p}}{ }^{35,36}$. For perfusion measurements, $K^{\text {trans }}$, describing the transfer rate of the used tracer from the blood plasma into the extravascular-extracellular space (EES), is the most valuable pharmacokinetic parameter related to the microvascular blood flow, vessel wall permeability and vessel density ${ }^{36}$. Malignancies presenting with a high $\mathrm{K}^{\text {trans }}$ value prior to treatment tend to better respond to chemo-(radio)-therapy treatment compared to tumors with relatively low values of $K^{\text {trans } 20-24,32-34}$.

The purpose of this study was to analyze the intratumoral FDG uptake and perfusion within rectal tumors before and after hypofractionated radiotherapy as well as to test a possible correlation between the tumor's metabolic activity and tumor perfusion. 


\subsection{Materials \& Methods}

\section{Patient Characteristics}

Patients diagnosed with non-locally advanced rectal cancer (NLARC) and scheduled for pre-operative radiotherapy treatment followed by surgery were considered for study enrollment. For each included patient, the TNM stage was determined from pretreatment magnetic resonance $(M R)$ images. All patients were pre-operatively referred to short course hypofractionated RT, five fractions of $5 \mathrm{~Gy}$ on five consecutive working days, followed by a total mesorectal excision (TME) within three days after the last RT fraction. According to the Dutch law, the medical ethics committee approved the trial and all patients gave written informed consent before entering the study.

\section{PET-CT and $\mathrm{PCT}$ acquisition}

All patients underwent a FDG-PET-CT scan combined with a PCT scan prior to the start of treatment and at the day of the last RT fraction. All PET-CT and PCT examinations were performed on the same dedicated Siemens TruePoint Biograph 40 PET-CT simulator (Siemens Medical, Erlangen, Germany). The patients were positioned equal to the radiotherapy treatment position using a laser alignment system to have minimal variations between imaging and treatment conditions and between the two imaging time-points. After a fasting period of at least 6 hours patients received an intravenous injection of FDG, with the activity normalized for the patients bodyweight (weight $[\mathrm{kg}] * 4+20[\mathrm{MBq}])$. Static PET acquisition was started after an uptake period of 60 minutes, with an acquisition time of 5 minutes per bed-position. For PET reconstruction (OSEM2D: 4 iterations, 8 subsets), CT based attenuation correction and 3D scatter- and decay-correction were performed. After the PET-CT scan, a pCT-scan was performed over 100 seconds. The field-of-view (FOV) for the pCT-scan was defined by an expert radiation oncologist (J.B. or G.L.) with knowledge of the PET-data. To ensure that the most representative tumor area was chosen, the tumor area with the highest FDG-uptake on the PET-scan was selected. Knowledge of the FOV selected for the first pCT-scan was used to select the identical region for the second pCT-scan. For the pCTscan, a volume of $120 \mathrm{ml}$ of an iodinated contrast-agent (300 mg iodine/ml, Xenetix 300, Guerbet, Aulnay-sous-Bois, France) was injected at a rate of $3 \mathrm{ml} / \mathrm{s}$ via an automatic injector (Stellant Sx, CT Injection System, MedRad, Warrendale, USA) into the antecubital fossa. The pCT-scan was performed in a static cine-mode over 12 contiguous slices with a slice thickness of $2.4 \mathrm{~mm}$, a FOV of $500 \mathrm{~mm}$ and an image size of $512 \times 512$ pixels. Other acquisition settings were: tube voltage $80 \mathrm{kVp}$, tube current 140 $\mathrm{mAs}$ and a rotation time of 1 second.

\section{PET Analysis \& Tumor contouring}

For each of the PET-scans, a tumor contour was generated using automated standardized-uptake-value (SUV) thresholding with the threshold (percentage of SUV $_{\max }$ within the tumor) depending on the tumor-to-background signal ratio with the gluteus muscle selected as relevant background ${ }^{37,38}$. Dedicated software (TrueD, Siemens Medical, Erlangen, Germany) was used to calculate the mean and maximum FDG uptake (SUV) within the tumor. Additionally, all PET-data were normalized for the blood glucose level (BGL) measured shortly before FDG administration ${ }^{19,39,40}$. 


\section{pCT Analysis}

Image-registration between the PET-CT scan and the PCT-scan was performed using a registration algorithm based on Mutual Information (Focal software, version 4.34, CMS Inc., St. Louis, Missouri). The automatic tumor delineation based on PET thresholding was exported and projected onto the PCT-dataset for further analysis. The PCT images were down-sampled from a voxel size of $0.98 \times 0.98 \times 2.4 \mathrm{~mm}$ to $3.92 \times 3.92 \times 4.8 \mathrm{~mm}$ to improve the signal-to-noise ratio (SNR). For the quantification of the dynamic pCTdata, the extended Kety-model was used, describing the uptake of a contrast agent from the blood plasma into the tissue by ${ }^{36}$ :

$$
C_{t}(t)=v_{p} C_{p}(t)+K^{\text {trans }} \int_{0}^{t} C_{p}(u) e^{\frac{-K^{\text {trans }}}{v_{e}}(t-u)} d u
$$

The blood plasma concentration curve $\left(C_{p}\right)$, extracted from the right external iliac artery, was derived from the acquired whole blood tracer concentration $\left(C_{b}\right)$ divided by (1-Hct), with the hematocrit value (Hct) set to $0.45^{36}$. To improve the SNR, $C_{p}$ was calculated by averaging the concentration time curves over all voxels selected inside the iliac artery. The tumor concentration curves $\left(C_{t}\right)$ were extracted from the dynamic PCT data on a voxel-by-voxel basis and on a tumor uptake curve based on the average of all tumor voxels. Pharmacokinetic analysis was performed using in-house developed software (MATLAB, R2008b, The Mathworks Inc., Natick, USA). The concentration time curves from pCT-data were fitted to the pharmacokinetic model, with the pharmacokinetic parameters being calculated using the Levenberg-Marquardt algorithm, with boundaries set to $0 \leq K^{\text {trans }} \leq 5 \mathrm{~min}^{-1}, 0 \leq v_{e} \leq 1$ and $0 \leq v_{p} \leq 1{ }^{41-43}$.

\section{Correlation of FDG uptake and tumor perfusion}

For each of the included patients, the mean and maximum FDG uptake (SUV) and transfer constant $\left(K^{\text {trans }}\right)$ within the tumor were correlated. Next, based on the tumor contour resulting from SUV-thresholding, the tumor was subdivided into eight subregions (Fig. 7.1). For each of the tumor sub-regions, the mean and maximum SUVS and $K^{\text {trans }}$ values were assessed and correlated. Furthermore, the mean FDG uptake in the voxels presenting with the lowest $25 \%$ of perfusion ( $K^{\text {trans }}$ values) was compared to the FDG uptake in the voxels with the $25 \%$ highest perfusion. To study early treatment effects, the metabolic activity of the tumors as well as the level of tumor perfusion were studied over time by comparing the pre- and post-treatment scans.

\section{Statistical Analysis}

Statistical differences between parameters were evaluated in SPSS (version 15.0, SPSS Inc., Chicago, IL, USA), performing a Wilcoxon-signed-rank test for the comparison of related measurements. Differences were considered to be significant when the $p$-value was less than 0.05 . For assessing the correlation between FDG uptake perfusion within the tumor, the Spearman's rank coefficient $(\rho)$ was calculated. 


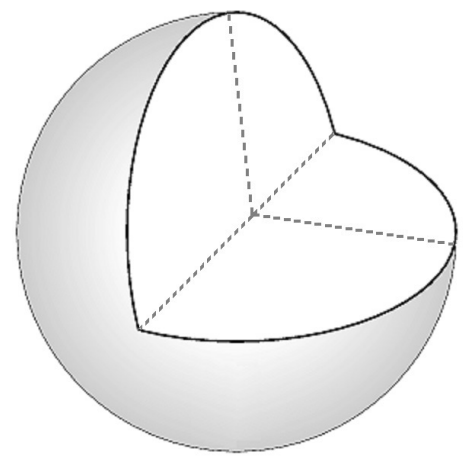

Figure 7.1: Spherical representation of a tumor with the dashed lines indicating the cutting-planes used for subdivision of the tumor into 8 sub-regions.

\subsection{Results}

\section{Patient Characteristics}

Twenty patients (mean age: $67.0 \pm 10.9$ years), diagnosed with NLARC were included in this study. Based on pre-treatment MR imaging (MRI), the clinical TNM staging was staged as T 1-3, N 0-1, M 0-1. For three of the included patients, large deformations of the rectum were observed when registering the CT- and PCT-scan. As these large deformations hamper an accurate registration of the PET-CT and pCT-scans, a reliable correlation of the FDG uptake within and perfusion of the tumor could not be ensured. For this reason, seventeen patients remained for further analysis.

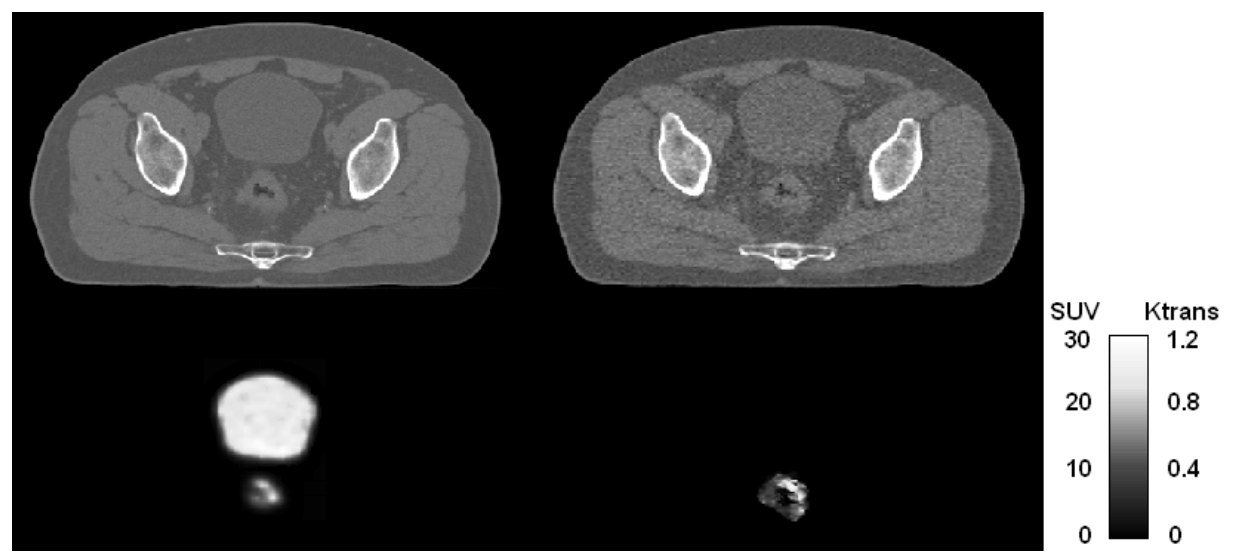

Figure 7.2: Pre-treatment comparison of a PET-CT image (left) and a perfusion-CT-image and the corresponding $K^{\text {trans }}$ map (right). 


\section{Correlation of the metabolic activity and perfusion of rectal tumors}

In figure 7.2, a FDG-PET-CT and pCT scan of a representative patient is shown. Visual inspection shows that there is a large correspondence of FDG uptake and perfusion within the tumor. Note the heterogeneous pattern of both the FDG uptake and perfusion of the tumor. When comparing the mean and maximum SUVs and $K^{\text {trans }}$ values within the tumors of the included patients, clear relationships were found with correlation coefficients $(\rho)$ of respectively $0.596(p=0.001)$ and $0.779(p<0.001)$ (Fig. 7.3). A location based analysis was performed by subdividing the tumors into eight subregions and comparing the FDG uptake and perfusion within these regions. The mean and maximum $K^{\text {trans }}$ values and SUVs showed a large correspondence, with positive correlation coefficients $(\rho)$ of respectively $0.413(p<0.001)$ and $0.540(p<0.001)$ (Fig. 7.4). Furthermore, the pre-treatment measured mean FDG uptake of the voxels within the tumor presenting with the $25 \%$ highest $K^{\text {trans }}$ values was found to be significantly higher compared to the FDG uptake of the voxels within the tumor presenting with the $25 \%$ lowest $K^{\text {trans }}$ values. For the $25 \%$ highest perfused tumor voxels, a $10.6 \pm 5.1 \%$ ( $p=$ 0.017 ) higher mean SUVs were found when compared to the $25 \%$ lowest perfused tumor voxels (Fig. 7.5).
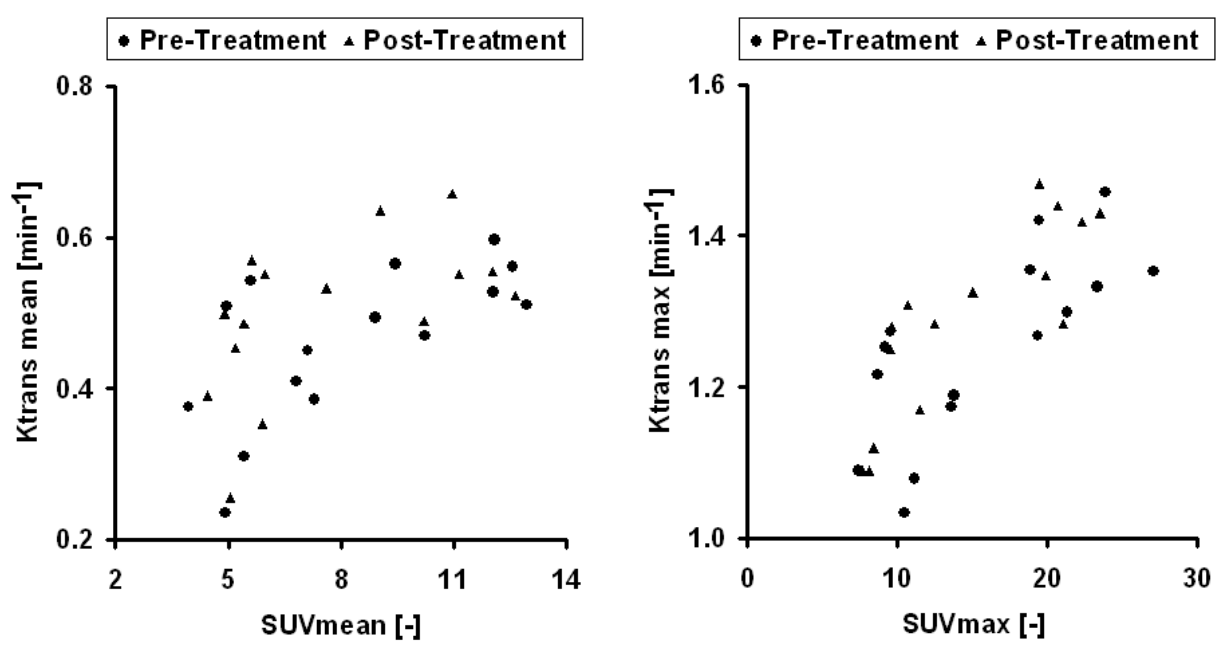

Figure 7.3: Scatter plots of the mean and maximum FDG uptake (SUV) within the tumor and the mean and maximum tumor perfusion ( $K^{\text {trans }}$ values). Note the positive correlation between the FDG uptake within and perfusion of tumor tissue for both the pre- and post-treatment situation. 

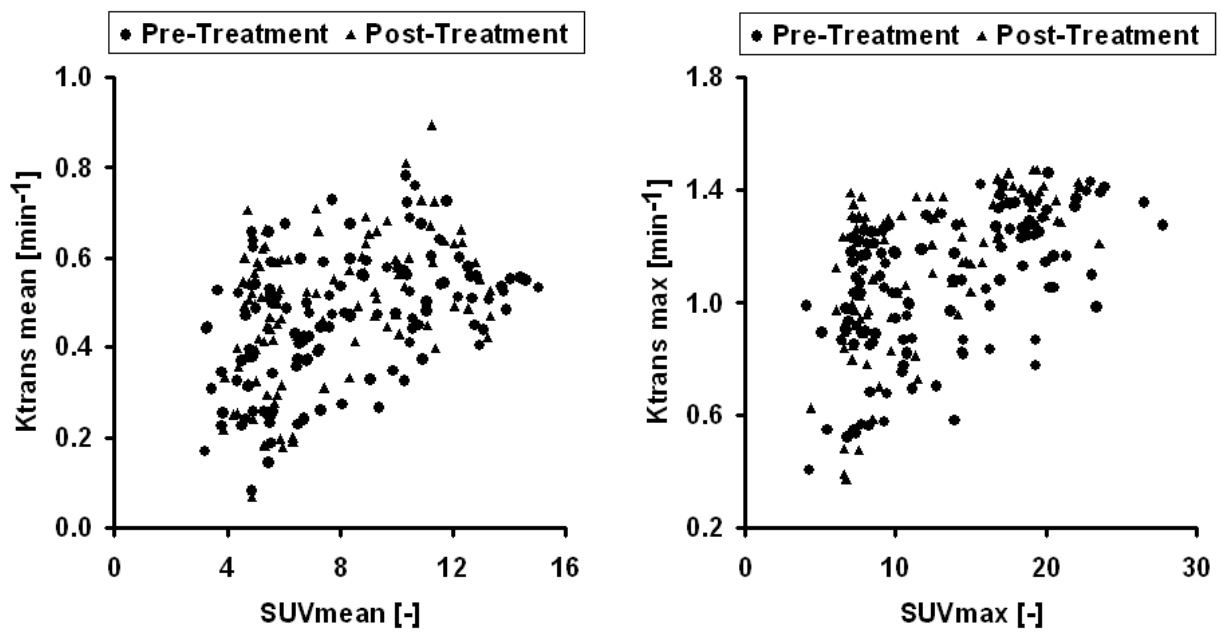

Figure 7.4: Scatter plots of the FDG uptake (SUV) and perfusion ( $K^{\text {trans }}$ values) within the 8 created tumor subregions for a location based analysis. Each dot or triangle represents the FDG uptake within and perfusion of one of the eight sub-regions created within a patient's rectal tumor respectively before and after treatment.

\section{Hypofractionated Radiotherapy Treatment Effect}

To study the early effects of hypofractionated radiotherapy on the FDG uptake within and perfusion of rectal tumors, PET-CT and PCT scans were performed prior to the start of treatment and at the day of the last radiotherapy fraction. When comparing the mean (pre: $7.9 \pm 3.0$, post: $7.5 \pm 2.8, p=0.362$ ) and maximum (pre: $15.8 \pm 6.4$, post: $14.6 \pm 5.8, p=0.280)$ SUVs, stable FDG uptake levels were found during short course hypofractionated RT ${ }^{44}$. However, for the mean (pre: $0.463 \pm 0.102 \mathrm{~min}^{-1}$, post: $0.500 \pm 0.105 \mathrm{~min}^{-1}, p=0.040$ ) and maximum (pre: $1.253 \pm 0.124 \mathrm{~min}^{-1}$, post: $1.340 \pm 0.092$ $\left.\min ^{-1}, p=0.003\right) K^{\text {trans }}$ values, significant increases were found between both pCT scans 7. In figure 7.6, the average histograms of the $K^{\text {trans }}$ values and SUVs within the tumor are shown for all patients. As can be seen from the histograms, pre-operative treatment with hypofractionated RT resulted in an increased tumor perfusion for the included patients, whereas stable FDG uptake levels were found. For the bins of the histogram presenting the number of voxels with a relatively high $K^{\text {trans }}$ value, an increase of the number of voxels was observed between the pre- and post-treatment pCT-scan. In contradiction, a decrease was found for the number of voxels in the bins with a lower $K^{\text {trans }}$ value. The shift of the histogram towards increased bins with relatively higher $K^{\text {trans }}$ indicates an increase of tumor perfusion due to pre-operative treatment with short course hypofractionated RT. 


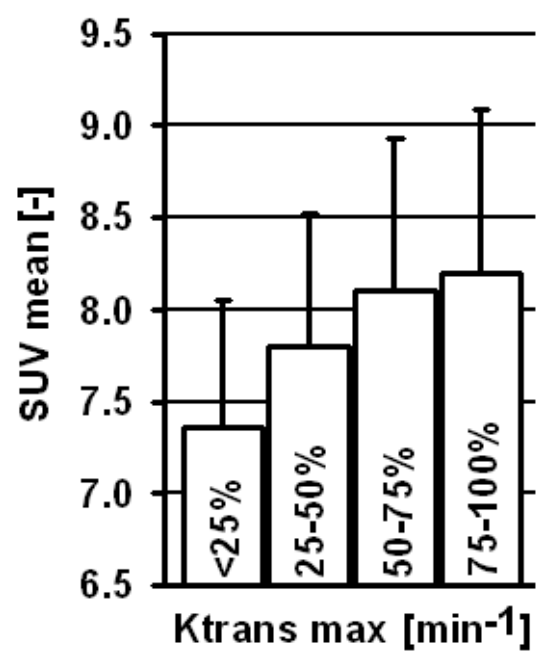

Figure 7.5: For the pre-treatment situation, a significant higher FDG uptake was observed within the tumor voxels presenting with the $25 \%$ highest $K^{\text {trans }}$ values when compared to the voxels presenting with the $25 \%$ lowest $K^{\text {trans }}$ values.
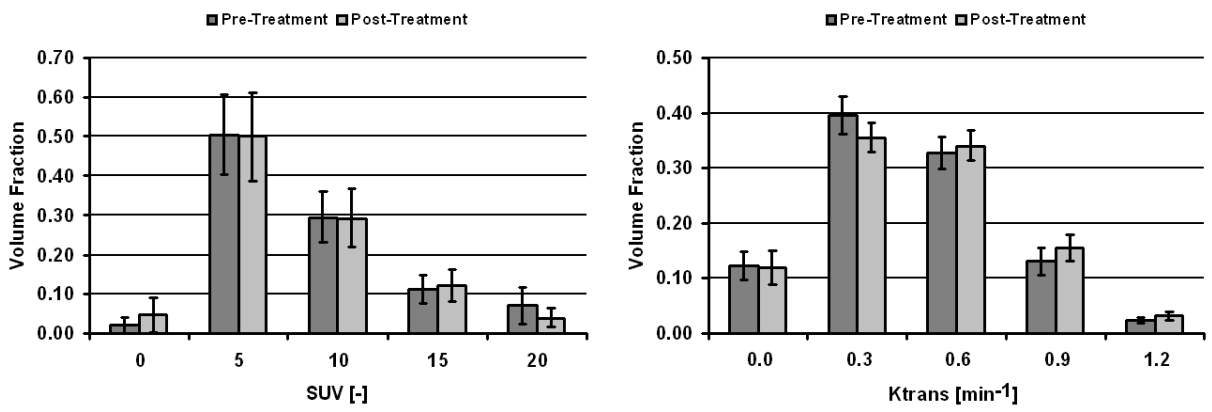

Figure 7.6: Treatment effect of hypofractionated radiotherapy on both the FDG uptake within and perfusion of rectal tumors. For the FDG uptake within rectal tumors, no significant treatment effect was observed, whereas a shift towards an overall better tumor perfusion was observed when evaluating the pre- and posttreatment Ktrans values.

\subsection{Discussion}

The purpose of this study was to analyze the intratumoral FDG uptake within and perfusion of rectal tumors before and after hypofractionated radiotherapy treatment as well as to test a possible correlation between FDG uptake and tumor perfusion. The FDG uptake within rectal tumors was found to positively correlate with tumor perfusion assessed from dynamic PCT-images. Highly perfused rectal tumors presented with higher FDG uptake levels compared to relatively low perfused tumors. To our knowledge, this manuscript is the first to describe a positive correlation of FDG-PET and 
pCT-imaging for rectal cancer. The presented findings for rectal cancer patients are in line with the findings of Miles et al. and Groves et al. for respectively lung and breast cancer patients ${ }^{25,27,28}$. Patients diagnosed with a relatively high perfused tumor tend to better respond to chemo-(radio)-therapy when compared to relatively low perfused tumors ${ }^{20-24,32,33}$. Based on the presented results it could be suggested that for highly perfused tumors a better distribution of the administered FDG and possibly also of a chemotherapeutic drug is possible. However, for different types of malignancies, FDG uptake has been studied as a surrogate marker for the detection and imaging of hypoxia, although with conflicting findings ${ }^{45-47}$. The results presented in this study, a strong positive correlation between FDG uptake and tumor perfusion, are in conflict with an increased FDG uptake as a surrogate marker of tumor hypoxia, since tumors are thought to develop hypoxia due to a low perfusion.

Another finding of this study was the difference in early treatment response as measured with respectively PET- and pCT-imaging. Early during hypofractionated RT, the mean and maximum FDG uptake levels within the tumors were found to be stable, whereas the mean and maximum $K^{\text {trans }}$ values were found to increase significantly. The significant increase of the mean and maximum $K^{\text {trans }}$ values early during pre-operative RT could be caused by endothelial cell death within the tumor, stress reactions of injured tumor cells or even death of tumor cells due to the hypofractionated RT treatment ${ }^{48}$. Endothelial cell death would result in endothelial cell leakage which in turn results in increased $K^{\text {trans }}$ values within the tumor.

Due to the relative small dimension of the FOV for PCT-imaging of the used PET-CT simulator in craniocaudal dimension, only $2.88 \mathrm{~cm}$, the craniocaudal coverage of the FOV resulted in some patients in incomplete tumor coverage. Due to possible incomplete tumor coverage, the calculated mean $K^{\text {trans }}$ values represent the perfusion of the tissue covered by the FOV. However, Goh et al. presented a study showing that an increase of the FOV in craniocaudal direction did not improve the reproducibility of perfusion measurements ${ }^{29}$. Nevertheless, nowadays state-of-the-art (PET-)CT scanners are becoming available which are able to perform volumetric perfusion measurement that encompass the entire tumor volume.

The major limitation of sequential functional imaging (PET- and pCT-imaging) followed by a voxel-wise comparison of the resulting images are deformations of the organs within the scanned region of the human body. Not only day-to-day differences in bladder and rectum filling, but also differences in bladder and rectum filling and bowel peristalsis within the short time-span (max. $20 \mathrm{~min}$ ) between the PET-CT and PCT-scan hampered an accurate voxel-wise comparison of the PET- and PCT-images. Because of this, for this study only a region-based comparison of FDG uptake within and perfusion of tumor tissue was performed. Even for the region-based correlation of the FDG uptake level and tumor perfusion, an accurate registration of the PET-CT and PCT-images should be ensured. Three of the included patients were excluded from further analysis because of large deformations of the rectum between the PET-CT and pCT scan.

Within a recent study, we presented pCT-imaging as an alternative modality to DCE-MRI imaging for the in-vivo evaluation of tumor perfusion in terms of the transfer constant $K^{\text {trans } 31}$. Because of the relatively high availability of CT-scanners over MRIscanners within radiotherapy departments, as well as the shorter total examination 
duration of a perfusion-CT scan, perfusion-CT imaging is an attractive alternative for DCE-MRI imaging. Also, perfusion-CT imaging has a better time-resolution compared to DCE-MRI imaging, which enables a high temporal acquisition of the first pass of the administered tracer. However, each perfusion-CT scan results in an additional effective dose of $15 \mathrm{mSv}$, whereas DCE-MR-imaging can be performed without ionizing radiation. However, all of the included patients were scheduled for pre-operative treatment with short course hypofractionated radiotherapy. The additional effective dose caused by perfusion-CT scanning should be compared to the much higher dose planned to preoperatively treat the patient, as well as to the relatively old age and the life expectations of the patients. For relatively young patients and/or when a MRI system is available, DCE-MRI is recommended over perfusion-CT imaging because of the better signalto-noise characteristics, stronger contrast uptake, larger tumor coverage and the absence of ionizing-radiation.

In conclusion, FDG uptake within the tumor, assessed with PET-imaging, was found to positively correlate with tumor perfusion assessed with dynamic perfusion-CT imaging. Highly perfused rectal tumors presented with higher FDG uptake levels compared to relatively low perfused tumors. Already early during hypofractionated radiotherapy, tumor perfusion was found to significantly increase, whereas FDG uptake levels were stable over time. Thus, for highly perfused rectal tumors, or regions within the tumor, a better distribution of the administered FDG is more likely compared to tumors with a relatively low perfusion. 


\subsection{References}

[1] Frykholm, G, Glimelius, B, Pahlman, L. Preoperative irradiation with and without chemotherapy (MFL) in the treatment of primarily non-resectable adenocarcinoma of the rectum. Results from two consecutive studies. Eur J Cancer Clin Oncol 1989;25:1535-1541.

[2] Sauer, R, Becker, H, Hohenberger, W, et al. Preoperative versus postoperative chemoradiotherapy for rectal cancer. N Engl J Med 2004;351:1731-1740.

[3] Sebag-Montefiore, D. Developments in the use of chemoradiotherapy in rectal cancer. Colorectal Dis 2006;8 Suppl 3:14-17.

[4] Dinter, DJ, Horisberger, K, Zechmann, C, et al. Can dynamic MR imaging predict response in patients with rectal cancer undergoing cetuximab-based neoadjuvant chemoradiation? Onkologie 2009;32:86-93.

[5] Hindie, E, Hennequin, C, Moretti, JL. Predicting response to chemoradiotherapy in rectal and oesophageal cancer with 18F-FDG: prognostic value and possible role in patient management. Eur J Nucl Med Mol Imaging 2007;34:1576-1582.

[6] de Geus-Oei, LF, Vriens, D, van Laarhoven, HW, van der Graaf, WT, Oyen, WJ. Monitoring and predicting response to therapy with 18F-FDG PET in colorectal cancer: a systematic review. J Nucl Med 2009;50 Suppl 1:43S-54S.

[7] Janssen, $\mathrm{MH}$, Aerts, HJ, Kierkels, RG, et al. Tumor perfusion increases during hypofractionated short course radiotherapy in rectal cancer: sequential perfusion-CT findings. Radiother Oncol 2010;94:156-160.

[8] Capirci, C, Rampin, L, Erba, PA, et al. Sequential FDG-PET/CT reliably predicts response of locally advanced rectal cancer to neo-adjuvant chemo-radiation therapy. Eur J Nucl Med Mol Imaging 2007;34:1583-1593.

[9] Capirci, C, Rubello, D, Chierichetti, F, et al. Long-term prognostic value of 18F-FDG PET in patients with locally advanced rectal cancer previously treated with neoadjuvant radiochemotherapy. AJR Am J Roentgenol 2006;187:W202-208.

[10] Vliegen, RF, Beets-Tan, RG, Vanhauten, B, et al. Can an FDG-PET/CT Predict Tumor Clearance of the Mesorectal Fascia after Preoperative Chemoradiation of Locally Advanced Rectal Cancer? Strahlenther Onkol 2008;184:457-464.

[11] Kalff, V, Duong, C, Drummond, EG, Matthews, JP, Hicks, RJ. Findings on 18F-FDG PET scans after neoadjuvant chemoradiation provides prognostic stratification in patients with locally advanced rectal carcinoma subsequently treated by radical surgery. J Nucl Med 2006;47:14-22.

[12] Amthauer, H, Denecke, T, Rau, B, et al. Response prediction by FDG-PET after neoadjuvant radiochemotherapy and combined regional hyperthermia of rectal cancer: correlation with endorectal ultrasound and histopathology. Eur J Nucl Med Mol Imaging 2004;31:811-819.

[13] Denecke, T, Rau, B, Hoffmann, KT, et al. Comparison of CT, MRI and FDG-PET in response prediction of patients with locally advanced rectal cancer after multimodal preoperative therapy: is there a benefit in using functional imaging? Eur Radiol 2005;15:1658-1666.

[14] Guillem, JG, Moore, HG, Akhurst, T, et al. Sequential preoperative fluorodeoxyglucose-positron emission tomography assessment of response to preoperative chemoradiation: a means for determining longterm outcomes of rectal cancer. J Am Coll Surg 2004;199:1-7.

[15] Melton, GB, Lavely, WC, Jacene, HA, et al. Efficacy of preoperative combined 18fluorodeoxyglucose positron emission tomography and computed tomography for assessing primary rectal cancer response to neoadjuvant therapy. J Gastrointest Surg 2007;11:961-969; discussion 969.

[16] Cascini, GL, Avallone, A, Delrio, P, et al. 18F-FDG PET is an early predictor of pathologic tumor response to preoperative radiochemotherapy in locally advanced rectal cancer. J Nucl Med 2006;47:1241-1248.

[17] Rosenberg, R, Herrmann, K, Gertler, R, et al. The predictive value of metabolic response to preoperative radiochemotherapy in locally advanced rectal cancer measured by PET/CT. Int J Colorectal Dis 2009;24:191-200. 
[18] Janssen, MH, Ollers, MC, Riedl, RG, et al. Accurate Prediction of Pathological Rectal Tumor Response after Two Weeks of Preoperative Radiochemotherapy Using (18)F-FluorodeoxyglucosePositron Emission Tomography-Computed Tomography Imaging. Int J Radiat Oncol Biol Phys 2010; 77:392-399.

[19] Janssen, MH, Ollers, MC, Stiphout, RG, et al. Blood glucose level normalization and accurate timing improves the accuracy of PET-based treatment response predictions in rectal cancer. Radiother Oncol 2010; 95: 203-208.

[20] Sahani, DV, Kalva, SP, Hamberg, LM, et al. Assessing tumor perfusion and treatment response in rectal cancer with multisection CT: initial observations. Radiology 2005;234:785-792.

[21] Bellomi, M, Petralia, G, Sonzogni, A, Zampino, MG, Rocca, A. CT perfusion for the monitoring of neoadjuvant chemotherapy and radiation therapy in rectal carcinoma: initial experience. Radiology 2007;244:486-493.

[22] Park, MS, Klotz, E, Kim, MJ, et al. Perfusion CT: noninvasive surrogate marker for stratification of pancreatic cancer response to concurrent chemo- and radiation therapy. Radiology 2009;250:110-117.

[23] Wang, J, Wu, N, Cham, MD, Song, Y. Tumor response in patients with advanced non-small cell lung cancer: perfusion CT evaluation of chemotherapy and radiation therapy. AJR Am J Roentgenol 2009;193:1090-1096.

[24] Zima, A, Carlos, R, Gandhi, D, Case, I, Teknos, T, Mukherji, SK. Can pretreatment CT perfusion predict response of advanced squamous cell carcinoma of the upper aerodigestive tract treated with induction chemotherapy? AJNR Am J Neuroradiol 2007;28:328-334.

[25] Groves, AM, Wishart, GC, Shastry, M, et al. Metabolic-flow relationships in primary breast cancer: feasibility of combined PET/dynamic contrast-enhanced CT. Eur J Nucl Med Mol Imaging 2009;36:416-421.

[26] Miles, KA. Perfusion CT for the assessment of tumour vascularity: which protocol? Br J Radiol 2003;76 Spec No 1:S36-42.

[27] Miles, KA, Griffiths, MR, Fuentes, MA. Standardized perfusion value: universal CT contrast enhancement scale that correlates with FDG PET in lung nodules. Radiology 2001;220:548-553.

[28] Miles, KA, Griffiths, MR, Keith, CJ. Blood flow-metabolic relationships are dependent on tumour size in non-small cell lung cancer: a study using quantitative contrast-enhanced computer tomography and positron emission tomography. Eur J Nucl Med Mol Imaging 2006;33:22-28.

[29] Goh, V, Halligan, S, Gartner, L, Bassett, P, Bartram, Cl. Quantitative colorectal cancer perfusion measurement by multidetector-row CT: does greater tumour coverage improve measurement reproducibility? Br J Radiol 2006;79:578-583.

[30] Harvey, C, Dooher, A, Morgan, J, Blomley, M, Dawson, P. Imaging of tumour therapy responses by dynamic CT. Eur J Radiol 1999;30:221-226.

[31] Kierkels, RG, Backes, WH, Janssen, MH, et al. Comparison Between Perfusion Computed Tomography and Dynamic Contrast-Enhanced Magnetic Resonance Imaging in Rectal Cancer. Int J Radiat Oncol Biol Phys 2010;77: 400-408.

[32] Hermans, R, Lambin, P, Van der Goten, A, et al. Tumoural perfusion as measured by dynamic computed tomography in head and neck carcinoma. Radiother Oncol 1999;53:105-111.

[33] Hermans, R, Van den Bogaert, W. Outcome prediction after surgery and chemoradiation of headand-neck squamous cell carcinoma (HNSCC), using baseline perfusion computed tomography (CT) microcirculatory parameters vs. tumor volume. Int J Radiat Oncol Biol Phys 2009;74:1307; author reply 1307.

[34] Hayano, K, Shuto, K, Koda, K, Yanagawa, N, Okazumi, S, Matsubara, H. Quantitative measurement of blood flow using perfusion CT for assessing clinicopathologic features and prognosis in patients with rectal cancer. Dis Colon Rectum 2009;52:1624-1629.

[35] Aerts, HJ, van Riel, NA, Backes, WH. System identification theory in pharmacokinetic modeling of dynamic contrast-enhanced MRI: influence of contrast injection. Magn Reson Med 2008;59:11111119.

[36] Tofts, PS, Brix, G, Buckley, DL, et al. Estimating kinetic parameters from dynamic contrastenhanced T(1)-weighted MRI of a diffusable tracer: standardized quantities and symbols. J Magn Reson Imaging 1999;10:223-232. 
[37] Daisne, JF, Sibomana, M, Bol, A, Doumont, T, Lonneux, M, Gregoire, V. Tri-dimensional automatic segmentation of PET volumes based on measured source-to-background ratios: influence of reconstruction algorithms. Radiother Oncol 2003;69:247-250.

[38] Ollers, M, Bosmans, G, van Baardwijk, A, et al. The integration of PET-CT scans from different hospitals into radiotherapy treatment planning. Radiother Oncol 2008;87:142-146.

[39] Beaulieu, S, Kinahan, P, Tseng, J, et al. SUV varies with time after injection in (18)F-FDG PET of breast cancer: characterization and method to adjust for time differences. J Nucl Med 2003;44:1044-1050.

[40] Eary, JF, Mankoff, DA. Tumor metabolic rates in sarcoma using FDG PET. J Nucl Med 1998;39:250254.

[41] Harrer, JU, Parker, GJ, Haroon, HA, et al. Comparative study of methods for determining vascular permeability and blood volume in human gliomas. J Magn Reson Imaging 2004;20:748-757.

[42] Jackson, A, Jayson, GC, Li, KL, et al. Reproducibility of quantitative dynamic contrast-enhanced MRI in newly presenting glioma. Br J Radiol 2003;76:153-162.

[43] Walker-Samuel, S, Leach, MO, Collins, DJ. Reference tissue quantification of DCE-MRI data without a contrast agent calibration. Phys Med Biol 2007;52:589-601.

[44] Janssen, $\mathrm{MH}$, Ollers, MC, van Stiphout, RG, et al. Evaluation of early metabolic responses in rectal cancer during combined radiochemotherapy or radiotherapy alone: sequential FDG-PET-CT findings. Radiother Oncol 2010;94:151-155.

[45] Burgman, P, Odonoghue, JA, Humm, JL, Ling, CC. Hypoxia-Induced increase in FDG uptake in MCF7 cells. J Nucl Med 2001;42:170-175.

[46] Dierckx, RA, Van de Wiele, C. FDG uptake, a surrogate of tumour hypoxia? Eur J Nucl Med Mol Imaging 2008;35:1544-1549.

[47] Busk, M, Horsman, MR, Jakobsen, S, Bussink, J, van der Kogel, A, Overgaard, J. Cellular uptake of PET tracers of glucose metabolism and hypoxia and their linkage. Eur J Nucl Med Mol Imaging 2008;35:2294-2303.

[48] de Lussanet QG, Backes WH, Griffioen AW. Dynamic contrast-enhanced magnetic resonance imaging of radiation induced therapy-induced microcirculation changes in rectal cancer. Int J Radiat Oncol Biol Phys 2005;63(5):1309-1315 


\section{Part III:}

Validation of automatic PETbased tumor delineation. 



\section{Chapter}

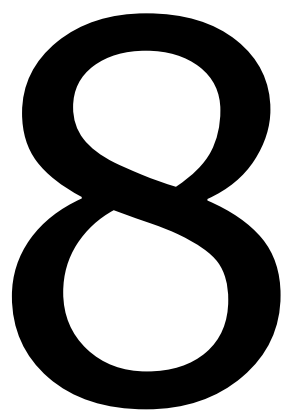

\section{Volumetric pathological validation of FDG-PET based automatic tumor delineation of rectal cancer.}

Marco Janssen

Marius Nap

Michel Öllers

Jørgen van den Bogaard

Robert Riedl

Jeroen Buijsen

Philippe Lambin

Guido Lammering 


\section{Abstract \\ Purpose}

The FDG uptake within a tumor, assessed with PET-imaging, can be used to automatically create a tumor contour using standardized uptake value (SUV) thresholding. The goal of this study was to validate the volume of an automatic generated PET-based tumor contour with pathology for rectal cancer.

\section{Materials \& Methods}

Patients diagnosed with non-locally advanced rectal cancer (NLARC), referred to preoperative treatment with short course hypofractionated radiotherapy (RT) were included in this study. Pre-operative treatment with RT was followed by a total mesorectal excision (TME) within 3 days after the last RT fraction. For each patient, a FDGPET-CT scan was performed prior to the start of treatment. The tumor was automatically delineated from the PET-images using SUV-thresholding, with the threshold depending on the tumor-to-background signal ratio. The volume of the PET-based tumor contour was validated by pathological examination of the resected specimen. For this validation, the resected specimen was sliced into $5 \mathrm{~mm}$ slices and each slice was photographed. On the photographs, the tumor was manually delineated by the pathologist. The tumor volume was calculated by multiplying the tumors surface area with the slice thickness of $5 \mathrm{~mm}$.

\section{Results}

Automatic SUV-threshold based tumor delineation resulted in an average tumor volume of $16.2 \pm 11.3 \mathrm{~cm}^{3}$ (range: 7.7 to $38.7 \mathrm{~cm}^{3}$ ), whereas pathological examination of the resected specimens resulted in an average tumor volume of $16.0 \pm 11.5 \mathrm{~cm}^{3}$ (range: 7.8 to $36.5 \mathrm{~cm}^{3}$ ). A correlation coefficient of $0.964(p<0.001)$ was found when correlating the tumor volumes resulting from PET-analysis and pathological examination.

\section{Conclusions}

The strong correlation between the tumor volumes resulting from SUV-thresholding of PET-images and pathological examination of the surgical specimen demonstrates the accuracy of automatic PET-based tumor delineation based on SUV-thresholding for patients diagnosed with rectal cancer. 


\subsection{Introduction}

For rectal cancer, pre-operative treatment with radiotherapy (RT), alone or in combination with chemotherapy has become the standard of care, most often followed by a total mesorectal excision (TME). Medical imaging modalities have become more and more important for the imaging, delineation and characterization of malignancies for treatment planning prior to the start of treatment as well as for treatment response evaluation during and after pre-operative treatment. In most cases, combined fluorodeoxyglucose positron-emission-tomography computed-tomography (FDG-PET-CT) imaging is used for visualization of the malignancy. The PET-CT images acquired prior to the start of pre-operative treatment are used to plan the highly energetic photon beams used for treatment of the tumor with RT. This, to ensure a sufficient radiation dose to the tumor while limiting the dose to surrounding healthy tissues. Where CTimaging provides only anatomical information about the location, orientation and size of the malignancy, FDG-PET-imaging has the added value of providing quantitative information about the metabolic activity of the malignancy, since viable tumor cells are known to avidly consume the glucose analog FDG.

The FDG uptake within the tumor, assessed with PET-imaging, can be used to automatically create a contour around the tumor ${ }^{1-9}$. Automatic tumor contouring on PET-images based on standardized uptake value (SUV) thresholding is most often used, with the threshold used for tumor delineation calculated from the tumor-tobackground signal ratio, known as the signal-to-background-ratio (SBR) method ${ }^{2,3,8,10}$. Manual tumor volume definition on CT-images is not only time consuming, but also subject to clinical interpretation, resulting in high levels of inter- and intra-observer variability ${ }^{11,12}$. PET-imaging was found to decrease the levels of inter- and intraobserver variability for the delineation of lung and rectal tumors ${ }^{11,13}$. Also, for lung, head and neck and rectal cancer, good correlations were found when comparing the tumor dimensions resulting from PET-analysis to the tumor dimensions from pathological analysis $1,2,6,8,11$. For rectal cancer, automatic tumor contouring by SUVthresholding was found to be superior to manual tumor delineation on respectively fused PET-CT images, CT images and MR images ${ }^{8}$. To our knowledge, only three studies yet presented a volumetric validation of PET-based automatic tumor contouring for head and neck, lung and rectal cancer ${ }^{1,2,6}$. However, the studies on lung and rectal cancer used a tumor contouring method based on gradients within the image using a watershed transform in combination with hierarchical clustering ${ }^{1,4,6}$. Also, the study on rectal cancer was performed on patients pre-operatively treated with long course chemo-radiotherapy $(C R T)$, resulting in significant tumor downsizing ${ }^{6}$. Only very few studies were yet presented on volumetric validation of automated SUVthresholding based tumor contouring with pathology ${ }^{1,2}$. The results showed a (nonsignificant) overestimation of the tumor volume when using SUV-thresholding based on the tumor-to-background signal ratio for automated delineation of the pharyngolaryngeal squamous cell carcinoma and non-small cell lung cancer. 
For modern RT-techniques like sub-volume boosting, non-uniform dose distributions, (high dose rate) brachytherapy or intra-operative radiotherapy (IORT), as well as for a reliable study on treatment related responses of the tumor, an accurate delineation of the tumor volume is indispensable ${ }^{14-21}$. Therefore, the aim of this study was to validate the volume of an automatic generated PET-based tumor contour with pathology for patients diagnosed with rectal cancer.

\subsection{Materials \& Methods}

\section{Patient Characteristics}

Patients diagnosed with non-locally advanced rectal cancer (NLARC) and scheduled for pre-operative treatment with hypofractionated RT were considered for study enrollment. The clinical TN staging was obtained from a pre-treatment magnetic resonance (MR) scan according to the TNM classification of malignant tumors (Edition 6). According to the national and regional treatment guidelines, patients with a T2N0-1 or a mid or high rectal T3NO-1 tumor with a predicted CRM of more than $2 \mathrm{~mm}$ were preoperatively treated with short course hypofractionated RT (5 fractions of 5 Gy on consecutive working days). A TME was planned within 3 days after the last RT-fraction. The Internal Review Board approved the study.

\section{FDG-PET-CT Image Acquisition and Processing}

For each of the included patients, a FDG-PET-CT scan was performed prior to the start of RT treatment. All PET-CT scans were performed on a dedicated Siemens Biograph 40 TruePoint PET-CT simulator (Siemens Medical, Erlangen, Germany) with an axial field of view of $16.2 \mathrm{~cm}$, slice thickness of $3 \mathrm{~mm}$ and a pixel spacing of $5.3456 \mathrm{~mm}$ in both directions. The scanner is equipped with ultra-fast detector electronics (Pico3D) and has a spatial resolution of approximately $6 \mathrm{~mm}$ full-width-at-half-maximum (FWHM). PET-imaging was done in $3 \mathrm{D}$, requiring a proper scatter correction. CT-based attenuation and decay correction was performed. PET images were reconstructed from the acquired list-mode (LM) data using Fourier-rebinning (FORE) and ordered-subsetexpectation-maximization-reconstruction (OSEM 2D) with 4 iterations and 8 subsets. After a fasting period of at least 6 hours prior to FDG injection, FDG was injected intravenously, with the activity normalized for the weight of the patient (weight $[\mathrm{kg}]$ * $4+20[\mathrm{MBq}])$, followed by injection of physiologic-saline $(10 \mathrm{ml})$. After an uptake period of 60 minutes, the patient was positioned on a flat tabletop using a movable laser alignment system in a "head-first supine" position with the arms crossed above the chest, equal to the treatment position. A PET-CT-scan of the abdominal region was performed using an acquisition time of 5 minutes per bed position. 


\section{FDG-PET Image Analysis}

From the pre-treatment performed FDG-PET-scan, a tumor contour was automatically generated using SUV iso-thresholding on dedicated software (TrueD VC60, Siemens Medical, Erlangen, Germany) (Fig. 8.1). The threshold used for contouring (percentage of SUV ${ }_{\max }$ within the tumor) was calculated from the tumor-to-background signal ratio with the gluteus muscle selected as a relevant background, using Equation $1^{3,10}$.

Threshold $=0.7813 \times\left(\frac{S U V_{\text {max tumor }}}{S U V_{\max \text { background }}}\right)^{-0.2988} \times 100[\%] \quad$ [Equation 1]

The volume of the tumor contour was validated by comparison with the tumor volume resulting from pathological examination of the surgical specimen.

\section{Pathological Processing of Surgical Specimens}

Immediately after resection, the specimen was send to the pathology department where it was opened, cleaned and put in a $4 \%$ formaldehyde solution for fixation purposes. After a 24 hour fixation process, the specimen was sliced using a meat slicer (slice thickness of $5 \mathrm{~mm}$ ) (Fig. 8.2). From each slice containing tumor, an image (Canon EOS 450D, Canon Inc., Tokyo, Japan) was made with a ruler within the image for dimension-reference. From the images, the tumor was delineated by a pathologist. In house developed software (MATLAB, R2010a, The Mathworks Inc., Natick, USA) was used to calculate the tumor volume based on the tumor contour drawn by the pathologist. First, for each of the images, the surface area $\left(\mathrm{cm}^{2}\right)$ of the tumor was calculated. The total surface area of the tumor was multiplied by the slice thickness of $5 \mathrm{~mm}$ to calculate the tumor volume $\left(\mathrm{cm}^{3}\right)$.

\section{Statistical Analysis}

The tumor volumes resulting from PET-analysis and pathological examination of the resected specimen were compared in SPSS (version 15.0, SPSS Inc., Chicago, IL, USA) by performing a Wilcoxon-signed-rank test. Differences were considered to be significant when the $p$-value was less than 0.05 . For correlation of the tumor volumes calculated from the PET-images and the surgical specimen, the Spearman's rank coefficient $(\rho)$ was calculated.

\subsection{Results}

\section{Patient Characteristics}

Eight patients, with a mean age of $66.6 \pm 6.3$ years (range: 56 to 76 years), diagnosed with NLARC were included in this study. Based on pre-treatment MR imaging (MRI), the clinical TNM staging was staged as T 2-3, N 0-1, M 0. 


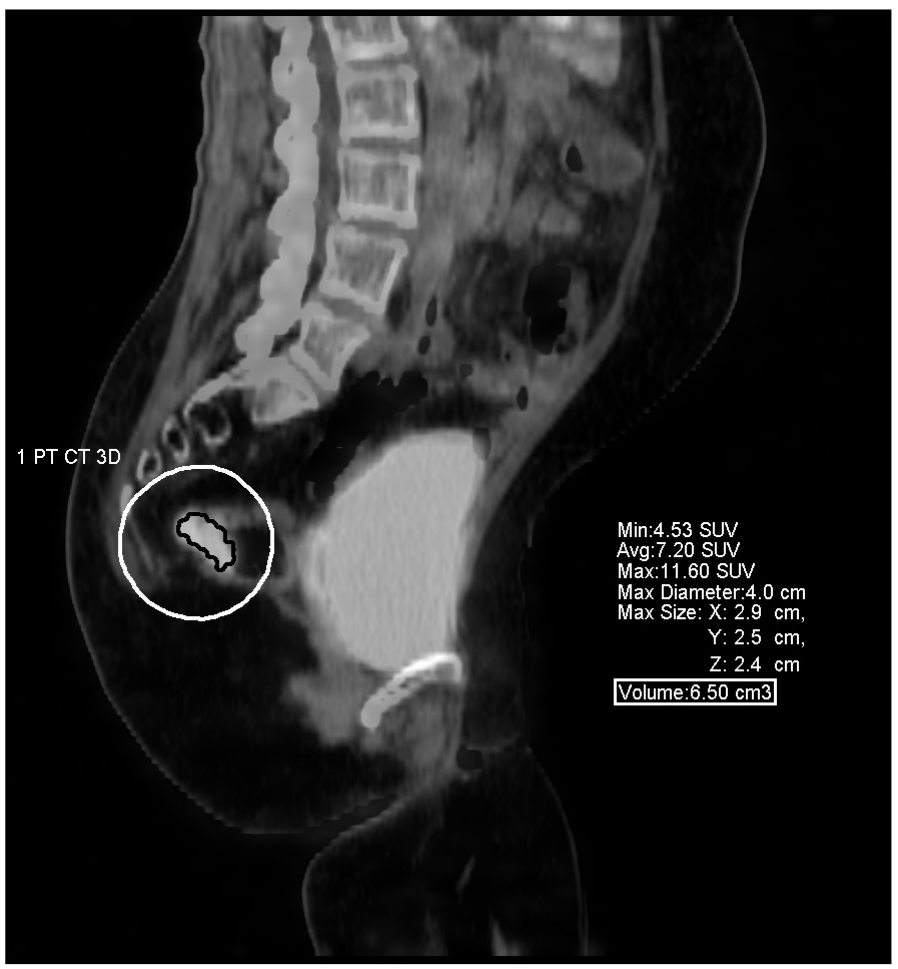

Figure 8.1: Sagittal plane of a fused FDG-PET-CT scan for a representative patient, with in white the manually selected region of interest around the tumor and in black the automatically created tumor contour resulting from SUV-iso-thresholding.

\section{Tumor Volume Analysis}

Automatic PET-based tumor delineation, based on SUV-iso-thresholding, resulted in an average tumor volume of $16.2 \pm 11.3 \mathrm{~cm}^{3}$ (range: 7.7 to $38.7 \mathrm{~cm}^{3}$ ). Pathological examination of the specimens resected from the patients by a TME after pre-operative treatment with short course hypofractionated RT resulted in an average tumor volume of $16.0 \pm 11.5 \mathrm{~cm}^{3}$ (range: 7.8 to $36.5 \mathrm{~cm}^{3}$ ). Comparison of the tumor volumes of PETbased tumor delineation and pathological examination of the resected specimen resulted in an average difference of $-3.6 \pm 13.7 \%$ (range: -20.0 to $16.2 \%)(p=0.829)$, which corresponds to a volume difference of $-0.2 \pm 2.0 \mathrm{~cm}^{3}$ (range: -2.2 to $3.0 \mathrm{~cm}^{3}$ ) (Fig. 8.3). A correlation coefficient of $0.964(p<0.001)$ was found when correlating the tumor volumes resulting from PET-analysis and pathological examination (Fig. 8.3). 


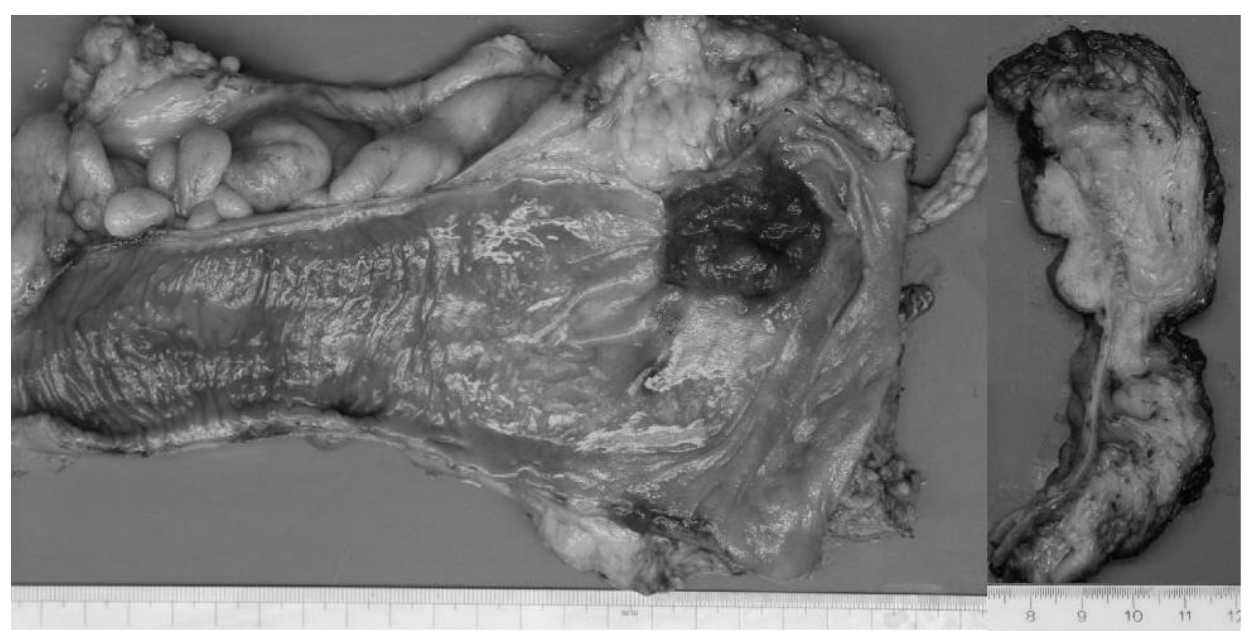

Figure 8.2: On the left side an image of a resected specimen after opening and cleaning and on the right side an image of one of the (tumor containing) slices of the specimen after slicing.

\section{口PET-Analysis 口Pathology}

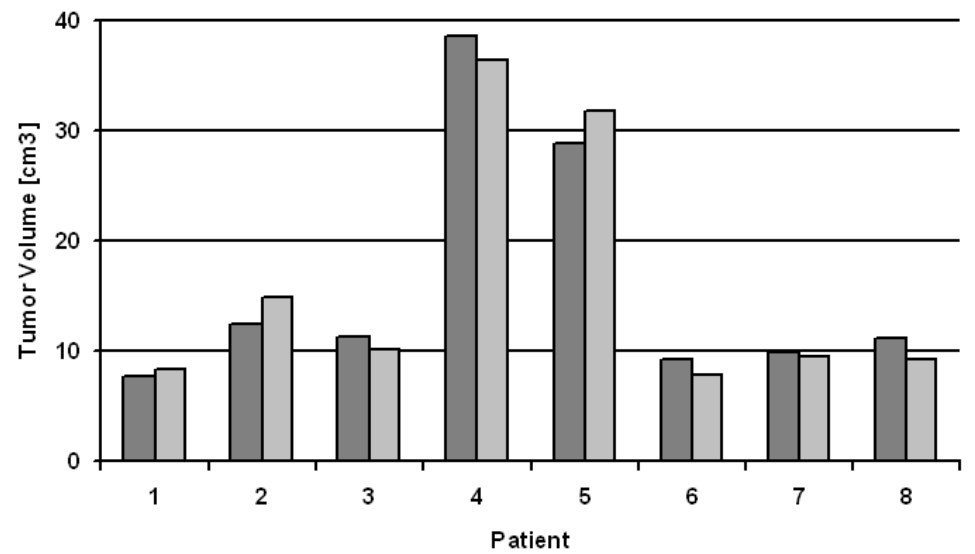

Figure 8.3: Bar-plot of the tumor volumes calculated from PET-analysis (dark grey) and pathological examination of the resected specimen (light grey). 


\subsection{Discussion}

This is the first study performing a pathological volumetric validation of automatic SUV thresholding tumor contouring for patients diagnosed with rectal cancer. The presented results prove that tumor delineation based on SUV-iso-thresholding yields an accurate delineation of the tumor volume. An accurate delineation of malignancies is indispensable for modern RT techniques as well as for investigation of treatment related responses of the tumor, especially since there is a growing interest in the use of sub-volume boosting techniques and non-uniform dose distributions ${ }^{14-21}$. Nowadays, combined FDG-PET-CT imaging is often used for visualization and characterization of malignancies prior to treatment. Automatic methods for tumor delineation on FDG-PET-images have been introduced over the last years ${ }^{1-9}$. Tumor contouring based on SUV-iso-thresholding is most often used, with the threshold depending on the tumor-to-background signal ratio 2, 3, 8, 10. However, SUV-thresholding based tumor contouring is dependent on the used PET-scanner as well as on the reconstruction algorithm and protocol ${ }^{3,10}$. The formula used for calculation of the SUV-threshold for automatic PET-based tumor contouring should be validated by a phantom study ${ }^{3,10}$. Also, for multi-centric studies, all used PET-scanners should be calibrated ${ }^{3,10}$.

No study yet demonstrated that the tumor volume automatically contoured from the FDG-PET-images by SUV-iso-thresholding matches the pathological tumor volume for rectal tumors. Over the last years, only a few studies have been published presenting correlations between the tumor dimensions measured from medical images and the tumor dimensions measured after resection of the tumor ${ }^{1,2,4-6,8,9,11}$. For lung and rectal cancer, good correlations were found when comparing the tumor diameter and tumor length resulting from PET-analysis to the tumor diameter and length resulting from pathological analysis ${ }^{8,11}$. For rectal cancer, the automatic PET-based contour was found to be superior to manual tumor delineation on respectively fused PET-CT images, CT images and MR images ${ }^{8}$. However, these studies only evaluated the length and maximal diameter of the tumor, without performing an analysis on the volume of the tumor.

For automated tumor contouring using SUV-thresholding, only very few studies were yet presented on volumetric validation for pharyngolaryngeal squamous cell carcinoma and non-small cell lung cancer, presenting a non-significant overestimation of the tumor volume when using SUV-thresholding for automated tumor delineation ${ }^{1}$, 2. To our knowledge only one study has yet been published performing a volumetric comparison between FDG-PET-imaging and pathology for rectal cancer ${ }^{6}$. However, the patients included in this study were pre-operatively treated with a long course of chemo-radiotherapy (CRT, 25-28 fractions of $1.8 \mathrm{~Gy}, 5$ days per week for 5 weeks, continuous infusion of 5 -fluorouracil $\left.\left(225 \mathrm{mg} / \mathrm{m}^{2}\right)\right)$. Imaging was performed at three time-points: before the start of CRT, after 10 fractions of RT and before surgery. Long course treatment with CRT is known to result in significant tumor downsizing and downstaging, with even a complete response in $15-30 \%$ of the patients ${ }^{22-27}$. From the 15 included patients, 6 presented with a pathological complete response and another 6 patients presented with a residual tumor volume of less than $1 \mathrm{~cm}^{3}$. Tumor shrinkage is also expected between the day of the last PET-CT scan and surgery. Also, the PET- 
based tumor volumes were determined using an algorithm based on gradients within the image, in stead of using SUV thresholding ${ }^{4}$.

For our study, the included patients were imaged with FDG-PET-CT prior to the start of pre-operative treatment with short course hypofractionated RT. A treatment related decrease of the PET-positive tumor volume was not expected for the included patients, since, in an earlier study, we observed stable PET-positive tumor volumes for NLARC patients during pre-operatively treated with short course hypofractionated RT by comparing the FDG-PET-CT scans performed prior to treatment and at the day of the last RT-fraction ${ }^{22}$.

When using FDG-PET-imaging for automatic tumor delineation, the partial-volumeeffect (PVE) should be kept in mind ${ }^{28}$. The PVE could result in an underestimation of the FDG uptake level within malignancies with a small volume, also influencing the contoured PET-positive tumor volume ${ }^{28}$. The PVE occurs for tumors smaller than three times the spatial resolution of the used PET-scanner ${ }^{28}$. The patients included in this study were diagnosed with NLARC, with relative small tumor volumes. However, for the patients included in this study, the smallest (PET-positive) tumor volume was 6.4 $\mathrm{cm}^{3}$. Therefore, we expected no significant influences of the PVE for the imaged malignancies.

After resection, the surgical specimens were fixated in formalin ( $4 \%$ formaldehyde solution). Theoretically, fixation of the specimen with formaldehyde could lead to a certain degree of shrinkage of the surgical specimen. However, no significant shrinkage effect was expected for the rectal tumors, because of the compact composition of the tumor and the fibrosis component in the region of around the tumor ${ }^{8,29}$.

In conclusion, this is the first study presenting a volumetric pathological validation of automatical SUV-threshold based tumor contouring for rectal cancer. The strong correlation between the tumor volumes resulting from SUV-thresholding of the PETimages and pathological examination of the surgical specimen demonstrates the accuracy of automatic PET-based tumor delineations based on SUV-iso-thresholding for patients diagnosed with rectal cancer. 


\subsection{References}

[1] Wanet, M., et al., Gradient-based delineation of the primary GTV on FDG-PET in non-small cell lung cancer: A comparison with threshold-based approaches, CT and surgical specimens. Radiother Oncol 2011;98:117-25.

[2] Daisne, J.F., et al., Tumor volume in pharyngolaryngeal squamous cell carcinoma: comparison at CT, MR imaging, and FDG PET and validation with surgical specimen. Radiology 2004;233:93-100.

[3] Daisne, J.F., et al., Tri-dimensional automatic segmentation of PET volumes based on measured source-to-background ratios: influence of reconstruction algorithms. Radiother Oncol 2003;69:247-50.

[4] Geets, X., et al., A gradient-based method for segmenting FDG-PET images: methodology and validation. Eur J Nucl Med Mol Imaging 2007; 34:1427-38.

[5] Visser, E.P., et al., Comparison of tumor volumes derived from glucose metabolic rate maps and SUV maps in dynamic 18F-FDG PET. J Nucl Med 2008;49:892-8.

[6] Roels, S., et al., Biological image-guided radiotherapy in rectal cancer: challenges and pitfalls. Int J Radiat Oncol Biol Phys 2009;75:782-90.

[7] Day, E., et al., A region growing method for tumor volume segmentation on PET images for rectal and anal cancer patients. Med Phys 2009; 36: 4349-58.

[8] Buijsen, J., et al., FDG-PET provides the best correlation with the tumor specimen compared to MRI and CT in rectal cancer. Radiother Oncol 2011;98:270-276.

[9] Yu, H.M., et al., Evaluation of gross tumor size using CT, 18F-FDG PET, integrated 18F-FDG PET/CT and pathological analysis in non-small cell lung cancer. Eur J Radiol 2009;72:104-13.

[10] Ollers, M., et al., The integration of PET-CT scans from different hospitals into radiotherapy treatment planning. Radiother Oncol 2008;87:142-6.

[11] van Baardwijk, A., et al., PET-CT-based auto-contouring in non-small-cell lung cancer correlates with pathology and reduces interobserver variability in the delineation of the primary tumor and involved nodal volumes. Int J Radiat Oncol Biol Phys 2007;68:771-8.

[12] Leunens, G., et al., Quality assessment of medical decision making in radiation oncology: variability in target volume delineation for brain tumours. Radiother Oncol 1993;29:169-75.

[13] Patel, D.A., et al., Impact of integrated PET/CT on variability of target volume delineation in rectal cancer. Technol Cancer Res Treat 2007;6: 31-6.

[14] Seierstad, T., et al., MR-guided simultaneous integrated boost in preoperative radiotherapy of locally advanced rectal cancer following neoadjuvant chemotherapy. Radiother Oncol 2009;93:279-84.

[15] Krishnan, S., et al., Phase II study of capecitabine (Xeloda) and concomitant boost radiotherapy in patients with locally advanced rectal cancer. Int J Radiat Oncol Biol Phys 2006;66:762-71.

[16] Petit, S.F., et al., Metabolic control probability in tumour subvolumes or how to guide tumour dose redistribution in non-small cell lung cancer (NSCLC): an exploratory clinical study. Radiother Oncol 2009;91:393-8.

[17] Petit, S.F., et al., [(18)F]fluorodeoxyglucose Uptake Patterns in Lung Before Radiotherapy Identify Areas More Susceptible to Radiation-Induced Lung Toxicity in Non-Small-Cell Lung Cancer Patients. Int J Radiat Oncol Biol Phys, 2010.

[18] Bentzen, S.M., Theragnostic imaging for radiation oncology: dose-painting by numbers. Lancet Oncol 2005;6:112-7.

[19] Bentzen, S.M., Dose painting and theragnostic imaging: towards the prescription, planning and delivery of biologically targeted dose distributions in external beam radiation oncology. Cancer Treat Res 2008;139: 41-62.

[20] Tanderup, K., D.R. Olsen, and C. Grau, Dose painting: art or science? Radiother Oncol 2006;79:245-8.

[21] Lambin, P., et al., The ESTRO Breur Lecture 2009. From population to voxel-based radiotherapy: exploiting intra-tumour and intra-organ heterogeneity for advanced treatment of non-small cell lung cancer. Radiother Oncol 2010;96:145-52.

[22] Janssen, M.H., et al., Evaluation of early metabolic responses in rectal cancer during combined radiochemotherapy or radiotherapy alone: sequential FDG-PET-CT findings. Radiother Oncol 2010;94:151-5. 
[23] Janssen, M.H., et al., Accurate prediction of pathological rectal tumor response after two weeks of preoperative radiochemotherapy using (18)F-fluorodeoxyglucose-positron emission tomography-computed tomography imaging. Int J Radiat Oncol Biol Phys 2010;77:392-9.

[24] Valentini, V., et al., Ten years of preoperative chemoradiation for extraperitoneal T3 rectal cancer: acute toxicity, tumor response, and sphincter preservation in three consecutive studies. Int J Radiat Oncol Biol Phys 2001;51:371-83.

[25] Capirci, C., et al., Sequential FDG-PET/CT reliably predicts response of locally advanced rectal cancer to neo-adjuvant chemo-radiation therapy. Eur J Nucl Med Mol Imaging 2007;34:1583-93.

[26] Capirci, C., et al., Long-term prognostic value of 18F-FDG PET in patients with locally advanced rectal cancer previously treated with neoadjuvant radiochemotherapy. AJR Am J Roentgenol 2006;187:W202-8.

[27] Vliegen, R.F., et al., Can an FDG-PET/CT predict tumor clearance of the mesorectal fascia after preoperative chemoradiation of locally advanced rectal cancer? Strahlenther Onkol 2008;184:457-64.

[28] Soret, M., S.L. Bacharach, and I. Buvat, Partial-volume effect in PET tumor imaging. J Nucl Med 2007; 48:932-45.

[29] Jonmarker, S., et al., Tissue shrinkage after fixation with formalin injection of prostatectomy specimens. Virchows Arch 2006;449:297-301. 



\section{Discussion}





\section{Chapter}

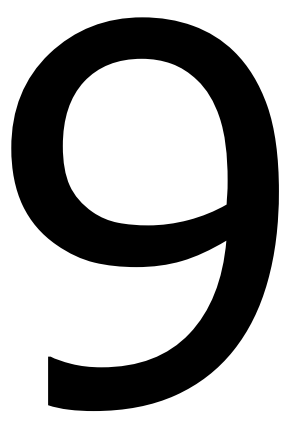

General discussion and future perspectives 
The objective of this thesis was to study treatment related changes of functional processes, the metabolic activity and perfusion, of rectal tumors during and after preoperative treatment. Also, the correlation between changes in different functional processes and between changes in the functional processes and the pathological treatment response were studied. This, to enable medical imaging based modifications of the treatment protocol, which can ultimately result in the development of individualized treatment approaches. In this chapter, the main findings are summarized and discussed with respect to relevant literature. Finally, future perspectives are presented to further improve the objective of patient specific treatment approaches for patients diagnosed with cancer.

\subsection{FDG-PET-CT based treatment response evaluation and prediction Response evaluation}

Non-invasive evaluation of the effects of treatment of malignancies, often based on fluorodeoxyglucose (FDG) positron emission tomography (PET) imaging, gives insight in tumor response to different treatment approaches. In this thesis, first evidence was provided that the reduction of the metabolic activity of rectal tumors during preoperative treatment might be induced by the chemotherapeutic agent (Capecitabine) or the combination of chemo- and radiotherapy (CRT) in stead of radiotherapy (RT) alone. Two patient groups were evaluated, treated with either short course hypofractionated RT or combined long course CRT. For the patients treated with CRT, a significant reduction of the metabolic activity of the tumor was observed already after the first week of treatment, whereas for patients treated with hypofractionated RT, stable FDG uptake levels were found, despite the higher biological equivalent dose (BED).

Additionally, a comparative study for a patient group treated with only chemotherapy would be useful to compare the metabolic treatment response to chemotherapy alone to the results of the patient group treated with CRT and RT alone. Unfortunately this was not possible, since pre-operative treatment of rectal cancer with only chemotherapy is not standard cure in our region. However, earlier studies proved that treatment with 5-Fluorouracil (5-FU) chemotherapy alone resulted in a consistent decrease of the FDG uptake of about $50 \%{ }^{1}$.

The patients included in the studies presented within this thesis were treated according to the national guidelines with either short course RT or long course CRT, based on their clinical TNM-stage and predicted circumferential resection margin (CRM), determined from magnetic resonance (MR) imaging. Patients with a T2N0-1 or a mid or high rectal T3NO-1 tumor with a predicted CRM of $>2 \mathrm{~mm}$ were preoperatively treated with short course hypofractionated RT, whereas patients with N2 disease, with low seated T3 tumors and patients with a tumor close to or invading the mesorectal fascia (CRM $<2 \mathrm{~mm}$ ) were treated with neoadjuvant CRT. The presented results on the metabolic treatment response early during pre-operative treatment with either long course CRT or short course hypofractionated RT were not expected to be biased by the non-random distribution of the patient population. The patients treated with CRT were diagnosed with locally advanced rectal cancer (LARC) with relatively high tumor volumes, whereas the patients treated with hypofractionated RT 
were diagnosed with non-locally advanced rectal cancer (NLARC). Larger, more advanced tumors are more likely to have hypoxic regions, resulting in an increased resistance to anti-cancer treatment ${ }^{2,3}$. So, a possible bias would have resulted in a higher metabolic treatment response for the patients diagnosed with NLARC treated with a higher BED compared to the larger tumors of the patients diagnosed with LARC and treated with a relatively low BED, which is opposite to our findings.

\section{Response prediction and validation}

For rectal cancer, several studies have been presented on the prediction of pathological treatment response based on FDG-PET-images performed prior to and after preoperative treatment ${ }^{4-12}$. However, only very few studies yet investigated the predictive strength of FDG-PET-imaging early during pre-operative treatment ${ }^{13-17}$.

An accurate prediction of the pathological tumor response early during preoperative treatment enables individualized treatment regimens with as goal further improvement of tumor response, a modified or delayed surgical approach or even cancellation of surgery in case of a predicted pathological complete response. In line with earlier findings for rectal and esophageal tumors, the studies performed in this thesis found FDG-PET-imaging after two weeks of pre-operative treatment with CRT to result in an accurate prediction of the pathological treatment response ${ }^{13-17}$. However, our study was, to our best knowledge, the first study to use FDG-PET-imaging at multiple time-points during pre-operative treatment: one and two weeks after the onset of therapy as well as prior to surgery. The presented results prove that the percent reduction of the maximal FDG uptake within rectal tumors after two weeks of CRT treatment results in the most accurate prediction of pathological response. A 48\% reduction of the maximum standardized-uptake-value $\left(S U V_{\max }\right)$ resulted in a specificity of $100 \%$ and a sensitivity of $64 \%$ when differentiating pathological responders (TRG 12) from pathological non-responders (TRG 3-5). The PET-data acquired after one week of treatment and prior to surgery resulted in less accurate predictions of the pathological treatment response, this in agreement with findings published earlier ${ }^{13,18}$.

Locally advanced rectal tumors pre-operatively treated with CRT show high levels of shrinkage during treatment. Most of the included patients presented with only small PET-positive residual tumor volumes at the FDG-PET-CT scan performed prior to surgery. An explanation for the less accurate prediction of the pathological tumor response based on pre-surgical performed PET-data could be due to the partial volume effect (PVE) ${ }^{19}$. The PVE could lead to an underestimation of the FDG uptake level in residual tumors with a small volume ${ }^{19}$. PET-images performed early during CRT are less influenced by the PVE due to less tumor shrinkage at the time of the PET-CT-scan.

Also, when predicting the pathological treatment response based on repeated FDG-PET-CT scans, the possible presence of peritumoral inflammatory responses should be kept in mind. Inflammatory cells are known to avidly consume FDG, which can lead to an underestimation of the metabolic response of the malignancy due to an increased FDG uptake in the peritumoral inflammatory tissue ${ }^{20,21}$. Based on only static PET-data it is very difficult to distinguish malignant tissue from inflammatory tissue. Studies on repeated FDG-PET-imaging at the same day or on dynamic FDG-PET-imaging reported a better distinctness of malignant and inflammatory tissue ${ }^{22,23}$. However, dynamic PET-imaging generally results in more discomfort for the patients and the 
analysis of dynamic PET-data is rather complex and time consuming ${ }^{24}$. A second limitation is that dynamic PET-imaging is possible for only 1 bedposition of the PETscanner, resulting in a limited field-of-view (FOV) of approximately $15-20 \mathrm{~cm}$. Also, motion of organs, due to for instance differences in bladder or rectal filling during the dynamic acquisition results in artifacts of the time activity curves (TACs) resulting from the dynamic PET-data.

Moreover, a high specificity of a predictive model should be preferred over a high sensitivity in order to avoid possible under-treatment of false positive classified patients. A false negative classification of a pathological responder would result in an over-treatment of the patient, whereas a false positive classification of a pathological non-responder would result in an under-treatment which can have serious consequences for the patient. For this study, patients with a tumor regression grade (TRG) of 1 or 2 according to the Mandard-criteria were defined as pathological responders and patients with a TRG 3-5 as pathological non-responders ${ }^{25}$. Earlier published literature proved patients with a TRG of 1-2 to have a better prognosis compared to patients with a TRG of 3-5, having less chance on local failure and an improved chance on metastasis- and disease free survival as well as an improved chance on overall survival 26, 27 . Also, an extended time interval between pre-operative treatment and surgery has been presented to result in more pronounced tumor regression and downstaging, whereas a shorter time interval may interrupt ongoing tumor necrosis ${ }^{28-30}$.

A PET-based response predictive model as presented in this thesis could in the near future be helpful to identify pathological responding patients (TRG 1-2), which could be referred to a less invasive surgical approach or even delayed or cancelled surgery, whereas non-responding patients (TRG 3-5) could for example be included in a radiotherapy boost trial using hypofractionation to improve the tumor response. However, validation of such PET-based response predictive models is required to ensure that the presented model is applicable for patients who are not included in the patient group on which the model is based. None of the PET-based models for the prediction of pathological response was yet properly validated for new included patients. Therefore, the predictive model presented in this thesis was validated for a patient group treated and imaged identical to the patients on which the model was based, resulting in a specificity of $93 \%$ and a sensitivity of $83 \%$.

\section{Quantification of PET-data}

The quantification of PET-images is known to be influenced by a diversity of factors, for instance the used equipment and protocol, the uptake time of the FDG and the patients blood glucose level (BGL) at the time of PET-imaging ${ }^{31-39}$. When quantifying (treatment related) changes in the FDG uptake level within a malignancy based on sequential FDG-PET-CT imaging, it is of high importance that all factors influencing the quantification should be kept constant ${ }^{40,41}$. When performing sequential FDG-PET-CTimaging within a single patient, especially when using automated tumor contouring methods based on SUV, it is important to use the same PET-CT scanner as well as identical imaging and reconstruction protocols for each of the examinations ${ }^{40-42}$.

The day before the planned FDG-PET-CT scan, the radiation technologist contacts the patient to explain the procedure of the FDG-PET-CT scan and to answer possible questions. The patient is asked for his or her body weight prior to each FDG-PET-CT 
scan. However, for an accurate quantification of (sequential) FDG-PET-data, it would be more accurate to measure the patient weight prior to the FDG-PET-CT scan than to rely on the information provided by the patient.

As FDG is a glucose analog which competes with normal glucose, this results in an inverse proportional correlation between the BGL of the patient at the time of FDG injection and the FDG uptake within tissues. However, none of the published studies on PET-based response predictions, except one, normalized the PET-data for the BGL of the patient at the time of the PET-scan ${ }^{43}$. For the patients included in the sequential FDG-PET-studies of this thesis, large inter- and intra-patient fluctuations of the BGL were found. The SUVs normalized for the BGL were found to be significantly different from the non-normalized SUVs, this in contrast to earlier findings ${ }^{43}$. For the patients included in the PET-based model for the prediction of the pathological treatment response, a significant increase of the area-under-the-curve (AUC) of ROC-curve analysis was found after normalization of the PET-data for the BGL of the patient. Within this study, BGL measurements were performed using an automatic device, as venous blood-sampling was not included in the study protocol. Therefore, analytical BGL measurements were not possible. However, the device used for BGLmeasurement (LifeScan One Touch, LifeScan Inc., Milpitas, USA) was proven to be the most accurate device, resulting in $100 \%$ of the measurements in the clinically accurate range ${ }^{44}$.

Also, FDG uptake was found to continue within rectal tumors at high rates even after an uptake period of 60 minutes, resulting in substantial SUV variations if time differences between FDG injection and the start of PET acquisition occur. Most published protocols on the prediction of treatment response based on PET-imaging use FDG uptake periods ranging from 40 to 60 minutes, whereas a delayed PET-acquisition, with a FDG uptake period of 90 minutes would significantly reduce the SUV time dependency. However, longer FDG uptake periods for patients are not preferable as this will ultimately decrease patient throughput on the PET-CT scanner.

\subsection{Perfusion-CT imaging during short course hypofractionated radio- therapy}

Perfusion changes during hypofractionated radiotherapy

During pre-operative treatment with short course hypofractionated RT, stable FDG uptake levels were found for patients diagnosed with NLARC, assessed from FDG-PETimaging performed prior to the start of treatment and at the day of the last RT fraction. However, from perfusion-CT (pCT) scans performed directly after the FDG-PET-CT scans, a significant increase of tumor perfusion was found early during pre-operative treatment with hypofractionated RT. The significant increase of tumor perfusion during the rather short time interval might be explained by first endothelial cell death, resulting in endothelial cell leakage which in turn results in increased perfusion values within the tumor ${ }^{45}$. An increase in tumor perfusion early during pre-operative treatment might improve the transport of cytotoxic agents of chemotherapy to the tumor, often administered during or after radiotherapy treatment. 
One of the major limitations of this study was the relatively small dimension of the field of view (FOV) of the PCT-scan in craniocaudal direction, resulting for some patients in an incomplete coverage of the tumor. For these patients, PCT measurements were obtained for the tumor region showing the highest FDG-uptake, as assessed from the static PET-CT scan. Due to incomplete tumor coverage, the calculated values of the three investigated pharmacokinetic parameters only represent the perfusion of the tumor region covered by the FOV. However, an earlier published study proved that an increased FOV in craniocaudal direction did not improve the reproducibility of perfusion measurements ${ }^{46}$. New generation CT-scanners have a FOV for perfusion-CT measurements of up to $48 \mathrm{~cm}$, ensuring complete tumor coverage.

Another limitation was that day-to-day differences in bladder and rectum filling hampered a voxel-wise comparison of the pharmacokinetic parameter maps of the pre- and post-treatment PCT-scans. Therefore, analysis was only performed on tumor level.

\section{FDG uptake correlates with tumor perfusion}

Highly perfused rectal tumors presented with higher FDG uptake levels compared to relatively low perfused tumors. The presented findings for rectal cancer patients are in line with the findings for lung and breast cancer patients ${ }^{47-49}$. For different types of malignancies, FDG uptake has been studied as a surrogate marker for the detection and imaging of hypoxia, although with conflicting findings ${ }^{50-52}$. The results of this study show that a strong positive correlation between FDG uptake and tumor perfusion is in conflict with increased FDG uptake as a surrogate marker of tumor hypoxia, since tumors are thought to develop hypoxia due to a low perfusion. Also, patients diagnosed with a relatively high perfused tumor tend to better respond to chemo-(radio)therapy when compared to relatively low perfused tumors, whereas hypoxia would result in an increased resistance to anti-cancer treatment ${ }^{53-57}$. Based on the presented results it could be suggested that for highly perfused tumors a better distribution of the administered FDG and possibly also of a chemotherapeutic drug is possible, improving the effects of anti-cancer treatment. However, further research to confirm these suggestions is necessary.

\subsection{Validation of automatic PET-based tumor delineation}

With a growing interest in the use of modern RT techniques like sub-volume boosting of the tumor, intra-operative radiotherapy (IORT) or (high dose rate) brachy-therapy, an accurate delineation of the tumor becomes indispensable ${ }^{58-65}$. Manual delineation of the tumor by a radiation oncologist based on CT, MR or even fused PET-CT images was found to result in high levels of inter- and intra-observer variability ${ }^{66-68}$.

However, from PET-images, it is possible to create a tumor contour fully automatically by SUV thresholding, with the threshold depending for example on the tumor-tobackground signal ratio ${ }^{69}$. Automated PET-based tumor delineation by SUV thresholding was found to show a strong correlation with pathology when comparing the tumor dimensions calculated from the PET-images and from pathology for head and neck, lung and rectal cancer ${ }^{66,70-74}$. For the most commonly used PET-based tumor contour- 
ing method, SUV thresholding based on the tumor-to-background signal ratio, only very few studies on volumetric validation have yet been performed with pathology, presenting a non-significant overestimation of the pathological tumor volume by SUVcontouring of pharyngolaryngeal squamous cell carcinoma and non-small cell lung cancer ${ }^{71,73}$. Two more studies have been presented on volumetric validations of PETbased tumor contouring ${ }^{72,73}$. However, these studies, performed for lung and rectal cancer, used a tumor contouring method based on image gradients instead of SUV thresholding ${ }^{75}$. The study on rectal cancer was performed for patients pre-operatively treated with long course CRT, known to result in a significant downsizing of the tumor, resulting in low volumes of the residual disease and even complete regression of the tumor in 6 of the 15 patients ${ }^{72,73}$.

For the patients included in this study, no tumor downsizing was expected between the pre-treatment FDG-PET-CT scan and surgery, since an earlier study on sequential FDG-PET-CT imaging for NLARC presented stable PET-positive tumor volumes during pre-operative treatment with short course hypofractionated $\mathrm{RT}^{76}$.

Automatic tumor delineation on PET-images should be handled with care for tumors with a small volume or follow-up FDG-PET-scans during or after pre-operative treatment with CRT, since the PVE could result in an underestimation of the FDG uptake level, also influencing the contoured PET-positive tumor volume ${ }^{19}$. The PVE occurs for tumors smaller than three times the spatial resolution of the used PETscanner ${ }^{19}$. The patients included in this study were diagnosed with NLARC with relative small tumor volumes. However, the smallest tumor volume for the included patients was $6.4 \mathrm{~cm}^{3}$. We therefore did not expect any significant influences of the PVE for the imaged rectal tumors.

Before pathological examination, the resected specimen was fixated in a $4 \%$ formaldehyde solution. However, no significant shrinkage effect was expected for the rectal tumors, because of the compact composition of the tumor and the fibrosis component in the region around the tumor ${ }^{70,77}$.

For the patients included in this study, automatic PET-based tumor delineation, based on SUV-iso-thresholding, resulted in an average tumor volume of $16.2 \pm 11.3 \mathrm{~cm}^{3}$ (range: 7.7 to $38.7 \mathrm{~cm}^{3}$ ), whereas pathological processing of the specimens resulted in an average tumor volume of $16.0 \pm 11.5 \mathrm{~cm}^{3}$ (range: 7.8 to $36.5 \mathrm{~cm}^{3}$ ). Comparison of the tumor volumes resulting from PET-analysis and pathological examination of the resected specimen resulted in an average difference $-0.2 \pm 2.0 \mathrm{~cm}^{3}$ (range: -2.2 to 3.0 $\mathrm{cm}^{3}$ ). The results of this study present a strong correlation between the tumor volumes resulting from automatic SUV-thresholding of PET-images and the tumor volumes resulting from pathological examination of the surgical specimen, demonstrating the accuracy of automatic tumor contouring based on SUV thresholding.

\subsection{Future Perspectives}

The results presented in this thesis form a solid basis for future research on the prediction of pathological treatment response. However, an overlap was observed in the PET-based metabolic response between pathological responders (TRG 1-2) and nonresponders (TRG 3-5) and between pathological complete responders (TRG 1) and 
good responders (TRG 2). A prediction of the pathological treatment response based on multiple factors could possibly improve the accuracy of the predictive model, with less overlap between the different patient groups. Multi-variate analysis based on clinical and imaging criteria could be useful to distinguish patients with a pathological complete response $(p C R)$ from patients with a pathological partial response (pPR) ${ }^{78}$. By the development of nomograms based on clinical and imaging criteria, the radiation oncologist and the surgeon are able to determine the patient specific probability of for instance $p C R$, disease free survival, metastasis free survival etc. ${ }^{78}$. For the patients with a predicted $\mathrm{PCR}$, with no visible residual disease on pre-surgical PET-CT and MRimages, the total mesorectal excision (TME) could be cancelled, whereas for patients with a predicted strong, but non-complete, pathological response, a less invasive, sphincter preserving, surgical approach (transanal endoscopic microsurgery (TEM)) could be used. On the other hand, for the patients with a predicted poor response, the treatment could be intensified if possible. The poor or non-responding patients could be included in a boost study with an increased radiotherapy dose to improve the local control rate. For patients with a (PET-positive) residual tumor, boosting techniques like dose painting can be used to intensify RT treatment to those parts of the tumor which are more resistant to anti-cancer treatment ${ }^{60}$.

In the future, the information assessed from functional imaging could be integrated into RT planning, resulting in non-uniform dose distributions based on intratumor heterogeneity. However, for malignancies in the abdomen, day to day differences in bladder or rectal filling can cause large differences in tumor location and orientation. Therefore, an accurate verification of the position of the tumor, between imaging and treatment conditions, should be ensured before non-uniform dose distributions with RT are possible in clinical practice. The use of markers in the direct neighborhood of the tumor increases the accuracy of image registration between the medical images used for treatment planning and the conebeam-CT images acquired shortly before RT-treatment, ultimately increasing the accuracy of the planned dose delivery. However, also (high dose rate) brachytherapy could possibly be used for boosting of (residual) rectal tumors with a relatively high radiotherapy dose ${ }^{79,80}$. Brachytherapy enables an increase of the radiation dose to the tumor without dramatically increasing the radiation dose to surrounding healthy tissues. Also, tumor motion due to day to day differences in bladder and rectal filling no longer influences the radiotherapy dose distribution when using brachytherapy, making a brachy-boost approach favourable over an external beam radiotherapy approach.

\subsection{Conclusions}

The objective of this thesis was to study treatment related changes in the FDG uptake within and perfusion of rectal tumors during pre-operative treatment with hypofractionated RT alone or combined CRT and to investigate the correlation between changes in these functional processes within the tumor and the pathological treatment response of the tumor. For the patients treated with CRT, a significant reduction of the metabolic activity of the tumor was observed already during treatment, whereas for 
patients treated with hypofractionated RT, stable FDG uptake levels were found, despite the higher biological equivalent dose (BED) of RT.

The percent reduction of FDG uptake within rectal tumors, measured two weeks after the onset of CRT treatment, was found to result in an accurate prediction of the pathological treatment response. An accurate prediction of the pathological response early during pre-operative treatment would enable for more individualized treatment regimens with as goal further improvement of tumor response, a modified or delayed surgical approach or even cancellation of the surgery in case of a predicted pathological complete response.

Accurate timing of PET-acquisition with identical FDG uptake times and correction of sequential FDG-PET-data for the patients BGL were found to increase the accuracy of PET-based models for the prediction of the pathological treatment response.

Where stable FDG uptake levels were observed early after pre-operative treatment with hypofractionated RT, a significant increase of tumor perfusion was assessed with perfusion-CT (pCT) imaging already at the day of the last fraction of hypofractionated RT. Also, a positive correlation between the metabolic activity (FDG uptake) and tumor perfusion was found.

An accurate delineation of the tumor is required to ensure a reliable study of treatment related responses of the tumor as well as for modern RT-techniques like sub-volume boosting, functional imaging based non-uniform dose distributions, intraoperative radiotherapy (IORT) or (high dose rate) brachytherapy. The strong correlation between the tumor volumes resulting from automatic tumor delineation on PETimages and pathological examination of the resected specimen demonstrates the accuracy of automatic tumor delineation using SUV thresholding for rectal cancer. 


\subsection{References}

[1] Direcks, W.G., et al., [18F]FDG and [18F]FLT uptake in human breast cancer cells in relation to the effects of chemotherapy: an in vitro study. Br J Cancer 2008;99:481-7.

[2] Khalil, A.A., S.M. Bentzen, and J. Overgaard, Steepness of the dose-response curve as a function of volume in an experimental tumor irradiated under ambient or hypoxic conditions. Int J Radiat Oncol Biol Phys 1997;39:797-802.

[3] Rofstad, E.K., et al., 31P NMR spectroscopy and $\mathrm{HbO2}$ cryospectrophotometry in prediction of tumor radioresistance caused by hypoxia. Int J Radiat Oncol Biol Phys 1989;16:919-23.

[4] Capirci, C., et al., Sequential FDG-PET/CT reliably predicts response of locally advanced rectal cancer to neo-adjuvant chemo-radiation therapy. Eur J Nucl Med Mol Imaging 2007;34:1583-93.

[5] Capirci, C., et al., Long-term prognostic value of 18F-FDG PET in patients with locally advanced rectal cancer previously treated with neoadjuvant radiochemotherapy. AJR Am J Roentgenol 2006;187:W202-8.

[6] Vliegen, R.F., et al., Can an FDG-PET/CT predict tumor clearance of the mesorectal fascia after preoperative chemoradiation of locally advanced rectal cancer? Strahlenther Onkol 2008;184:457-64.

[7] Kalff, V., et al., Findings on 18F-FDG PET scans after neoadjuvant chemoradiation provides prognostic stratification in patients with locally advanced rectal carcinoma subsequently treated by radical surgery. J Nucl Med 2006;47:14-22.

[8] Amthauer, H., et al., Response prediction by FDG-PET after neoadjuvant radiochemotherapy and combined regional hyperthermia of rectal cancer: correlation with endorectal ultrasound and histopathology. Eur J Nucl Med Mol Imaging 2004;31:811-9.

[9] Denecke, T., et al., Comparison of CT, MRI and FDG-PET in response prediction of patients with locally advanced rectal cancer after multimodal preoperative therapy: is there a benefit in using functional imaging? Eur Radiol 2005;15:1658-66.

[10] Guillem, J.G., et al., Sequential preoperative fluorodeoxyglucose-positron emission tomography assessment of response to preoperative chemoradiation: a means for determining longterm outcomes of rectal cancer. J Am Coll Surg 2004;199:1-7.

[11] Melton, G.B., et al., Efficacy of preoperative combined 18-fluorodeoxyglucose positron emission tomography and computed tomography for assessing primary rectal cancer response to neoadjuvant therapy. J Gastrointest Surg 2007;11:961-9; discussion 969.

[12] Capirci, C., et al., The role of dual-time combined 18-fluorodeoxyglucose positron emission tomography and computed tomography in the staging and restaging workup of locally advanced rectal cancer, treated with preoperative chemoradiation therapy and radical surgery. Int J Radiat Oncol Biol Phys 2009;74:1461-9.

[13] Cascini, G.L., et al., 18F-FDG PET is an early predictor of pathologic tumor response to preoperative radiochemotherapy in locally advanced rectal cancer. J Nucl Med 2006;47:1241-8.

[14] Rosenberg, R., et al., The predictive value of metabolic response to preoperative radiochemotherapy in locally advanced rectal cancer measured by PET/CT. Int J Colorectal Dis 2009;24:191-200.

[15] Hindie, E., C. Hennequin, and J.L. Moretti, Predicting response to chemoradiotherapy in rectal and oesophageal cancer with 18F-FDG: prognostic value and possible role in patient management. Eur J Nucl Med Mol Imaging 2007;34:1576-82.

[16] de Geus-Oei, L.F., et al., Monitoring and predicting response to therapy with 18F-FDG PET in colorectal cancer: a systematic review. J Nucl Med 2009;50 Suppl 1:43S-54S.

[17] Lambrecht, M., et al., The use of FDG-PET/CT and diffusion-weighted magnetic resonance imaging for response prediction before, during and after preoperative chemoradiotherapy for rectal cancer. Acta Oncol 2010;49:956-63.

[18] Wieder, H.A., et al., Time course of tumor metabolic activity during chemoradiotherapy of esophageal squamous cell carcinoma and response to treatment. J Clin Oncol 2004;22:900-8.

[19] Soret, M., S.L. Bacharach, and I. Buvat, Partial-volume effect in PET tumor imaging. J Nucl Med 2007;48:932-45.

[20] Kao, P.F., Y.H. Chou, and C.W. Lai, Diffuse FDG uptake in acute prostatitis. Clin Nucl Med 2008;33:308-10. 
[21] Larson, S.M., Cancer or inflammation? A Holy Grail for nuclear medicine. J Nucl Med 1994;35:1653-5.

[22] Kumar, R., et al., Potential of dual-time-point imaging to improve breast cancer diagnosis with (18)F-FDG PET. J Nucl Med 2005;46:1819-24.

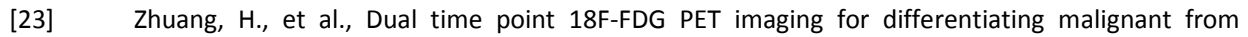
inflammatory processes. J Nucl Med 2001;42:1412-7.

[24] Cheebsumon, P., et al., Measuring response to therapy using FDG PET: semi-quantitative and full kinetic analysis. Eur J Nucl Med Mol Imaging 2011;38:832-42.

[25] Mandard, A.M., et al., Pathologic assessment of tumor regression after preoperative chemoradiotherapy of esophageal carcinoma. Clinicopathologic correlations. Cancer 1994;73:2680-6.

[26] Rodel, C., et al., Prognostic significance of tumor regression after preoperative chemoradiotherapy for rectal cancer. J Clin Oncol 2005;23:8688-96.

[27] Vecchio, F.M., et al., The relationship of pathologic tumor regression grade (TRG) and outcomes after preoperative therapy in rectal cancer. Int J Radiat Oncol Biol Phys 2005;62:752-60.

[28] Veenhof, A.A., et al., The relationship of histological tumor regression grade (TRG) and two different time intervals to surgery following radiation therapy for locally advanced rectal cancer. Int J Colorectal Dis 2009; 24:1091-6.

[29] Habr-Gama, A., et al., Interval between surgery and neoadjuvant chemoradiation therapy for distal rectal cancer: does delayed surgery have an impact on outcome? Int J Radiat Oncol Biol Phys 2008;71:1181-8.

[30] Dolinsky, C.M., et al., Effect of time interval between surgery and preoperative chemoradiotherapy with 5-fluorouracil or 5-fluorouracil and oxaliplatin on outcomes in rectal cancer. J Surg Oncol 2007;96:207-12.

[31] Beaulieu, S., et al., SUV varies with time after injection in (18)F-FDG PET of breast cancer: characterization and method to adjust for time differences. J Nucl Med 2003;44:1044-50.

[32] Keyes, J.W., Jr., SUV: standard uptake or silly useless value? J Nucl Med 1995; 36:1836-9.

[33] Visser, E.P., O.C. Boerman, and W.J. Oyen, SUV: from silly useless value to smart uptake value. J Nucl Med 2010;51:173-5.

[34] Vriens, D., et al., Methodological considerations in quantification of oncological FDG PET studies. Eur J Nucl Med Mol Imaging 2010;37:1408-25.

[35] Lindholm, P., et al., Influence of the blood glucose concentration on FDG uptake in cancer--a PET study. J Nucl Med 1993;34:1-6.

[36] Langen, K.J., et al., The influence of plasma glucose levels on fluorine-18-fluorodeoxyglucose uptake in bronchial carcinomas. J Nucl Med 1993; 34: 355-9.

[37] Adams, M.C., et al., A systematic review of the factors affecting accuracy of SUV measurements. AJR Am J Roentgenol 2010;195:310-20.

[38] Boellaard, R., Standards for PET image acquisition and quantitative data analysis. J Nucl Med 2009; 50 Suppl 1:11S-20S.

[39] Shankar, L.K., et al., Consensus recommendations for the use of 18F-FDG PET as an indicator of therapeutic response in patients in National Cancer Institute Trials. J Nucl Med 2006;47:1059-66.

[40] Boellaard, R., et al., The Netherlands protocol for standardisation and quantification of FDG whole body PET studies in multi-centre trials. Eur J Nucl Med Mol Imaging 2008;35:2320-33.

[41] Boellaard, R., et al., FDG PET and PET/CT: EANM procedure guidelines for tumour PET imaging: version 1.0. Eur J Nucl Med Mol Imaging 2010;37: 181-200.

[42] Ollers, M., et al., The integration of PET-CT scans from different hospitals into radiotherapy treatment planning. Radiother Oncol 2008;87:142-6.

[43] Stahl, A., et al., Comparison of different SUV-based methods for monitoring cytotoxic therapy with FDG PET. Eur J Nucl Med Mol Imaging 2004; 31:1471-8.

[44] Brunner, G.A., et al., Validation of home blood glucose meters with respect to clinical and analytical approaches. Diabetes Care 1998;21:585-90.

[45]. de Lussanet, Q.G., et al., Dynamic contrast-enhanced magnetic resonance imaging of radiation therapy-induced microcirculation changes in rectal cancer. Int J Radiat Oncol Biol Phys 2005;63:1309-15. 
[46] Goh, V., et al., Quantitative colorectal cancer perfusion measurement by multidetector-row CT: does greater tumour coverage improve measurement reproducibility? Br J Radiol 2006;79:57883.

[47] Groves, A.M., et al., Metabolic-flow relationships in primary breast cancer: feasibility of combined PET/dynamic contrast-enhanced CT. Eur J Nucl Med Mol Imaging 2009;36:416-21.

[48] Miles, K.A., M.R. Griffiths, and M.A. Fuentes, Standardized perfusion value: universal CT contrast enhancement scale that correlates with FDG PET in lung nodules. Radiology 2001;220:548-53.

[49] Miles, K.A., M.R. Griffiths, and C.J. Keith, Blood flow-metabolic relationships are dependent on tumour size in non-small cell lung cancer: a study using quantitative contrast-enhanced computer tomography and positron emission tomography. Eur J Nucl Med Mol Imaging 2006;33:22-8.

[50] Burgman, P., et al., Hypoxia-Induced increase in FDG uptake in MCF7 cells. J Nucl Med 2001;42:170-5.

[51] Dierckx, R.A. and C. Van de Wiele, FDG uptake, a surrogate of tumour hypoxia? Eur J Nucl Med Mol Imaging 2008;35:1544-9.

[52] Busk, M., et al., Cellular uptake of PET tracers of glucose metabolism and hypoxia and their linkage. Eur J Nucl Med Mol Imaging 2008;35:2294-303.

[53] Sahani, D.V., et al., Assessing tumor perfusion and treatment response in rectal cancer with multisection CT: initial observations. Radiology 2005; 234:785-92.

[54] Bellomi, M., et al., CT perfusion for the monitoring of neoadjuvant chemotherapy and radiation therapy in rectal carcinoma: initial experience. Radiology 2007;244:486-93.

[55] Park, M.S., et al., Perfusion CT: noninvasive surrogate marker for stratification of pancreatic cancer response to concurrent chemo- and radiation therapy. Radiology 2009;250:110-7.

[56] Wang, J., et al., Tumor response in patients with advanced non-small cell lung cancer: perfusion CT evaluation of chemotherapy and radiation therapy. AJR Am J Roentgenol 2009;193:1090-6.

[57] Zima, A., et al., Can pretreatment CT perfusion predict response of advanced squamous cell carcinoma of the upper aerodigestive tract treated with induction chemotherapy? AJNR Am J Neuroradiol 2007;28:328-34.

[58] Seierstad, T., et al., MR-guided simultaneous integrated boost in preoperative radiotherapy of locally advanced rectal cancer following neoadjuvant chemotherapy. Radiother Oncol 2009;93:279-84.

[59] Krishnan, S., et al., Phase II study of capecitabine (Xeloda) and concomitant boost radiotherapy in patients with locally advanced rectal cancer. Int J Radiat Oncol Biol Phys 2006;66:762-71.

[60] Petit, S.F., et al., Metabolic control probability in tumour subvolumes or how to guide tumour dose redistribution in non-small cell lung cancer (NSCLC): an exploratory clinical study. Radiother Oncol 2009;91:393-8.

[61] Petit, S.F., et al., [(18)F]fluorodeoxyglucose Uptake Patterns in Lung Before Radiotherapy Identify Areas More Susceptible to Radiation-Induced Lung Toxicity in Non-Small-Cell Lung Cancer Patients. Int J Radiat Oncol Biol Phys, 2010.

[62] Bentzen, S.M., Theragnostic imaging for radiation oncology: dose-painting by numbers. Lancet Oncol 2005;6:112-7.

[63] Bentzen, S.M., Dose painting and theragnostic imaging: towards the prescription, planning and delivery of biologically targeted dose distributions in external beam radiation oncology. Cancer Treat Res 2008;139:41-62.

[64] Tanderup, K., D.R. Olsen, and C. Grau, Dose painting: art or science? Radiother Oncol 2006;79:245-8.

[65] Lambin, P., et al., The ESTRO Breur Lecture 2009. From population to voxel-based radiotherapy: exploiting intra-tumour and intra-organ heterogeneity for advanced treatment of non-small cell lung cancer. Radiother Oncol 2010;96:145-52.

[66] van Baardwijk, A., et al., PET-CT-based auto-contouring in non-small-cell lung cancer correlates with pathology and reduces interobserver variability in the delineation of the primary tumor and involved nodal volumes. Int J Radiat Oncol Biol Phys 2007;68:771-8.

[67] Leunens, G., et al., Quality assessment of medical decision making in radiation oncology: variability in target volume delineation for brain tumours. Radiother Oncol 1993;29:169-75.

[68] Patel, D.A., et al., Impact of integrated PET/CT on variability of target volume delineation in rectal cancer. Technol Cancer Res Treat 2007;6: 31-6. 
[69] Daisne, J.F., et al., Tri-dimensional automatic segmentation of PET volumes based on measured source-to-background ratios: influence of reconstruction algorithms. Radiother Oncol 2003;69:247-50.

[70] Buijsen, J., et al., FDG-PET provides the best correlation with the tumor specimen compared to MRI and CT in rectal cancer. Radiother Oncol 2011;98:270-6.

[71] Daisne, J.F., et al., Tumor volume in pharyngolaryngeal squamous cell carcinoma: comparison at CT, MR imaging, and FDG PET and validation with surgical specimen. Radiology 2004;233:93-100.

[72] Roels, S., et al., Biological image-guided radiotherapy in rectal cancer: challenges and pitfalls. Int J Radiat Oncol Biol Phys 2009;75:782-90.

[73] Wanet, M., et al., Gradient-based delineation of the primary GTV on FDG-PET in non-small cell lung cancer: A comparison with threshold-based approaches, CT and surgical specimens. Radiother Oncol 2011;98:117-25.

[74] Yu, H.M., et al., Evaluation of gross tumor size using CT, 18F-FDG PET, integrated 18F-FDG PET/CT and pathological analysis in non-small cell lung cancer. Eur J Radiol 2009;72:104-13.

[75] Geets, X., et al., A gradient-based method for segmenting FDG-PET images: methodology and validation. Eur J Nucl Med Mol Imaging 2007; 34:1427-38.

[76] Janssen, M.H., et al., Evaluation of early metabolic responses in rectal cancer during combined radiochemotherapy or radiotherapy alone: sequential FDG-PET-CT findings. Radiother Oncol 2010;94:151-5.

[77] Jonmarker, S., et al., Tissue shrinkage after fixation with formalin injection of prostatectomy specimens. Virchows Arch 2006;449:297-301.

[78] van Stiphout, R.G., et al., Development and external validation of a predictive model for pathological complete response of rectal cancer patients including sequential PET-CT imaging. Radiother Oncol 2011; 98: 126-33.

[79] Tunio, M.A., et al., High-dose-rate intraluminal brachytherapy during preoperative chemoradiation for locally advanced rectal cancers. World J Gastroenterol 2010;16:4436-42.

[80] Sun Myint, A., et al., Can increasing the dose of radiation by HDR brachytherapy boost following pre operative chemoradiotherapy for advanced rectal cancer improve surgical outcomes? Colorectal Dis 2010;12 Suppl 2:30-6. 



\section{Summary}


In Europe, rectal cancer is one of the most common malignancies. Rectal cancer can be divided into non-locally advanced rectal cancer (NLARC) and locally advanced rectal cancer (LARC), based on the margin between the tumor and the mesorectal fascia (MRF). For NLARC, short course hypofractionated radiotherapy (RT), followed by surgery with a total mesorectal excision (TME), is the treatment of choice, whereas LARC is most often treated with combined long course chemo-radiotherapy (CRT), also followed by a TME.

Prior to treatment, (multi-modality) medical imaging is often used to obtain information about the location, size and stage of the tumor. Nowadays, a combination of anatomical imaging with computed tomography (CT) and functional imaging with positron emission tomography (PET) and/or perfusion-CT ( characterization of malignancies. For PET-imaging, fluorodeoxyglucose (FDG) is most often chosen as PET-tracer. The concentration of the FDG represents the metabolic activity of the imaged tissue, often quantified in standardized uptake values (SUVS).

In this thesis, the potential of functional imaging for the evaluation of treatment related tumor responses was investigated. The correlation between changes in the different functional processes was studied, as well as the correlation between changes in the functional processes and the pathological treatment response. This, to study the possibility of medical imaging based modifications of the treatment protocol, which can ultimately result in the development of patient specific treatment approaches.

\section{Part I: FDG-PET-CT based treatment response evaluation and predic- tion}

In Chapter 2 we studied the metabolic treatment response of rectal tumors to preoperative treatment with respectively short course hypofractionated RT or combined long course CRT. For the patients treated with hypofractionated RT, two FDG-PET-CT scans were performed: prior to the start of RT and at the day of the fifth RT fraction, whereas for the patients treated with CRT, FDG-PET-CT imaging was performed prior to the start of treatment and one week after the onset of CRT. For the patients referred to pre-operative treatment with CRT, a significant reduction of the FDG uptake level was observed already one week after the onset of treatment, whereas preoperative treatment with RT alone did not result in significant changes of the metabolic activity of rectal tumors, despite the higher applied RT dose.

In Chapter 3 of this thesis, the metabolic treatment response of tumors preoperatively treated with CRT was correlated to the pathological treatment response evaluated from the resected specimen. To study the best PET-based predictor of the pathological treatment response, FDG-PET-CT imaging was performed at multiple time points during and after pre-operative treatment: once prior to the start of treatment, one and two weeks after the onset of treatment and prior to surgery. 
The percent decrease of the maximum FDG uptake $\left(S U V_{\max }\right)$ within rectal tumors after two weeks of pre-operative treatment with CRT was found to result in the most accurate prediction of the tumor regression grade (TRG). A cut-off threshold of $43 \% \mathrm{SUV}_{\max }$ decrease resulted in a sensitivity of $77 \%$ and a specificity of $93 \%$.

Since the quantification of PET-data is known to be dependent on many factors, the influence of changes in the FDG uptake period as well as fluctuations in the patient's blood glucose level (BGL) on PET-based predictions of the pathological treatment response are studied in Chapter 4. For the patient's BGL, measured shortly before FDG injection, strong (day-to-day) inter- and intra-patient fluctuations were found. When correlating the percent reduction of SUV $\mathrm{V}_{\max } 2$ weeks after the onset of CRT with the pathological treatment response, a significant increase in the area under the curve (AUC) of ROC-curve analysis was found when normalizing the PET-data for the measured BGLs, indicating an increase of the predictive strength of the response predictive model. Also, a SUV ${ }_{\max }$ time-dependency of $1.30 \pm 0.66$ every $10 \mathrm{~min}$. (range: 0.39 to 2.58) was found during the first 60 minutes of PET-acquisition. These results strongly underline the necessity of BGL-normalization of (sequential) FDG-PET-data and a precise time-management between FDG-injection and the start of PET-acquisition when using sequential FDG-PET-CT imaging for PET-based predictions of the pathological treatment response.

For further development and clinical usefulness of PET-based response predictive models, a validation of the model, with patients not included in the patient group on which the model is based, should be performed. In Chapter 5, the PET-based model for the prediction of the pathological treatment response, as presented in Chapter 3 and 4 , was validated with a secondary patient group, treated and imaged identical to the patients on which the predictive model was based. A cut-off value of $48 \% \mathrm{SUV}_{\max }$ decrease two weeks after the onset of treatment resulted in a sensitivity and specificity of respectively 64 and $100 \%$ for the initial patient group, and a sensitivity and specificity of 83 and $93 \%$ for the second (validation) patient group, with one pathological non-responding patient being false positively predicted as a pathological responder.

\section{Part II: Perfusion-CT imaging during short course hypofractionated radiotherapy}

In Chapter 2, no significant metabolic treatment response was observed early after pre-operative treatment with short course hypofractionated RT. Therefore, in Chapter 6 of this thesis, the perfusion of non-locally advanced rectal tumors was studied during treatment with hypofractionated RT using PCT imaging. Twenty-three patients diagnosed with NLARC were included, which underwent PCT-imaging prior to and after pre-operative treatment with hypofractionated RT. Perfusion was quantified with three pharmacokinetic parameters using the extended Kety-model: $K^{\text {trans }}, v_{e}$ and $v_{p}$. Perfusion differences between tumor and muscle tissue and changes of perfusion over time were evaluated for each patient. Overall, the median $K^{\text {trans }}$ values were signifi- 
cantly higher for tumor than for muscle tissue on the pre-treatment scan as well as on the post-treatment scan. For tumor tissue, the median $K^{\text {trans }}$ values significantly increased from the pre-treatment to the post-treatment situation, whereas for muscle tissue stable perfusion levels were found.

In Chapter 7, the intratumoral FDG uptake and perfusion of rectal tumors were studied prior to and after hypofractionated RT to study possible correlations between the two imaging modalities. All patients underwent FDG-PET-CT and PCT-imaging prior to the start of hypofractionated RT and at the day of the last RT-fraction. The mean and maximum FDG-uptake (SUV), assessed from PET-imaging, and the transfer constant $\left(K^{\text {trans }}\right)$, assessed from pCT-imaging, were correlated. Also, the tumor was subdivided into 8 sub-regions. For each sub-region, the mean and maximum SUVS and $K^{\text {trans }}$ values were assessed and correlated. Furthermore, the mean FDG-uptake in voxels presenting with the lowest $25 \%$ of perfusion was compared to the FDG uptake in the voxels with the $25 \%$ highest perfusion. The mean and maximum $K^{\text {trans }}$ values positively correlated with the corresponding SUVs $(\rho=0.596, p=0.001$ and $\rho=0.779, p<0.001)$. Positive correlations were also found for the $K^{\text {trans }}$ values and SUVS within the sub-regions of the tumor (mean: $\rho=0.413, p<0.001$ and max: $\rho=0.540, p<0.001$ ). Also, the mean FDG uptake in the $25 \%$ highest perfused tumor regions was significantly higher compared to the $25 \%$ lowest perfused regions $(10.6 \pm 5.1 \%, p=0.017)$.

\section{Part III: Validation of automatic PET-based tumor delineation}

In Chapter $\mathbf{8}$ of this thesis, the automatically generated tumor contour resulting from SUV-thresholding of PET-images was validated with the tumor volume calculated from pathological examination of the specimen resected from the patient after preoperative treatment with hypofractionated RT. Automatic SUV-thresholding tumor delineation resulted in an average tumor volume of $16.2 \pm 11.3 \mathrm{~cm}^{3}$ (range: 7.7 to 38.7 $\mathrm{cm}^{3}$ ), whereas pathological analysis of the resected specimens resulted in an average tumor volume of $16.0 \pm 11.5 \mathrm{~cm}^{3}$ (range: 7.8 to $36.5 \mathrm{~cm}^{3}$ ), with a correlation coefficient of $0.964(p<0.001)$. Comparison of the tumor volumes resulted in an average difference of $-0.2 \pm 2.0 \mathrm{~cm}^{3}$ (range: -2.2 to $3.0 \mathrm{~cm}^{3}$ ). The strong correlation between the tumor volumes resulting from SUV-thresholding and pathological examination of the surgical specimen demonstrates the accuracy of automated PET-based tumor delineations for rectal cancer. 


\section{Samenvatting}


In Europa is endeldarmkanker één van de meest voorkomende vormen van kanker. Endeldarmkanker wordt onderverdeeld in het lokaal beperkt endeldarmcarcinoom (non-locally advanced rectal cancer (NLARC)) en het lokaal gevorderd endeldarmcarcinoom (locally advanced rectal cancer (LARC)) op basis van de marge tussen de tumor en de mesorectale fascia. NLARC wordt meestal behandeld met hypogefractioneerde radiotherapie (RT), gevolgd door chirurgie (totale mesorectale excisie (total mesorectal excision, TME)), terwijl LARC behandeld wordt met een combinatie van langdurige chemo-radiotherapie (CRT), ook gevolgd door een TME.

Voor aanvang van behandeling wordt medische beeldvorming gebruikt om informatie te verkrijgen over de locatie, afmeting en stagering van de tumor. Tegenwoordig wordt vaak een combinatie van anatomische beeldvorming (computed tomography (CT), magnetic resonance imaging (MRI)) en functionele beeldvorming (positron emission tomography (PET), perfusie-CT ( $\mathrm{pCT})$ ) gebruikt om de tumor te karakteriseren. Voor PET-beeldvorming wordt binnen de oncologie meestal gebruik gemaakt van een glucose-analoog (fluorodeoxyglucose (FDG)) als tracer. De concentratie van het FDG weerspiegelt de metabole activiteit van de weefsels, vaak weergegeven als een gestandaardiseerde opname waarde (standardized uptake value (SUV)).

In dit proefschrift worden de toepassingen en mogelijkheden van functionele beeldvorming voor de evaluatie van behandeling gerelateerde veranderingen binnen de tumor onderzocht. Veranderingen van diverse processen binnen de tumor werden onderling gecorreleerd, maar ook werd de correlatie gevonden met de pathologische respons. Dit is noodzakelijk, om de mogelijkheid en nauwkeurigheid van medische beeldvorming gebaseerde responsvoorspellingen te onderzoeken, welke uiteindelijk kunnen bijdragen in de ontwikkeling van patiënt specifieke behandelplannen.

\section{Deel I: FDG-PET-CT gebaseerde respons evaluatie en predictie}

In Hoofdstuk 2 hebben we de verandering in metabole activiteit van endeldarmtumoren bestudeerd gedurende pre-operatieve behandeling met hypogefractioneerde RT en gecombineerde CRT. Voor de patiënten behandeld met hypogefractioneerde RT werden twee FDG-PET-CT scans gemaakt: één keer voor de start van behandeling en één keer op de dag van de vijfde en laatste radiotherapie fractie, terwijl voor de patiënten behandelt met gecombineerde CRT, FDG-PET-CT beeldvorming verkregen werd voor aanvang van behandeling en na de eerste week van behandeling. Een statistisch significante afname van het FDG opname niveau binnen de tumor werd gevonden voor de patiënten die preoperatief behandeld werden met gecombineerde $\mathrm{CRT}$, terwijl pre-operatieve behandeling met hypogefractioneerde RT niet resulteerde in sigificante veranderingen in de metabole activiteit van de tumoren, ondanks de hogere radiotherapie dosis.

In Hoofdstuk 3 van dit proefschrift hebben we de metabole respons van de tumor gedurende pre-operatieve behandeling met langdurige CRT gecorreleerd aan de pathologische respons bepaald uit het pathologische preparaat verkregen na chirurgie. 
Ter bestudering van de meest nauwkeurige voorspelling van de pathologische respons zijn er voor iedere patiënt FDG-PET-CT beelden gemaakt op meerdere tijdstippen: voorafgaande, gedurende en na afloop van de pre-operatieve behandeling met CRT. De FDG-PET-CT beelden werden verkregen op de volgende tijdstippen: voor aanvang van de behandeling, na afloop van de eerste en tweede behandelweek en kort voor de geplande chirurgie. Het percentage afname van de maximale FDG opname binnen de tumor $\left(\mathrm{SUV}_{\max }\right)$ na twee weken van behandeling resulteerde in de meest nauwkeurige predictie van de pathologische respons. Een afkapwaarde van $43 \%$, om de pathologische responders te onderscheiden van de pathologische niet-responders, resulteerde in een sensitiviteit van $77 \%$ en een specificiteit van $93 \%$.

De quantificatie van FDG-PET-data is afhankelijk van een groot aantal factoren. De invloed van fluctuaties in zowel de opname tijd van het FDG als ook de bloedglucosespiegel van de patiënt op de nauwkeurigheid van PET-gebaseerde respons voorspellingen zijn bestudeerd in Hoofdstuk 4. Voor de patiënten binnen dit onderzoek werden sterke inter- en intra-patiënt fluctuaties waargenomen voor de bloedglucosespiegels gemeten kort voor injectie van het FDG. Normalisatie van de herhaalde PET-data voor de gemeten bloedglucosespiegel resulteerde in een significante toename in de oppervlakte onder de ROC-curve in vergelijking met de niet genormaliseerde PET-data. Een voorspelling van de pathologische respons, gebaseerd op PET-data verkregen na twee weken van behandeling, werd significant nauwkeuriger wanneer de PET-data genormaliseerd werd voor de gemeten bloedglucosespiegel. Ook werd er een gemiddelde tijdsafhankelijkheid van $1.30 \pm 0.66$ per 10 minuten ( 0.39 tot 2.58 ) gevonden voor de bepaling van de FDG opname in de tumor gedurende de eerste 60 minuten na FDG injectie. Deze resultaten benadrukken de noodzakelijkheid van gestandaardiseerde PET-protocollen wanneer herhaalde PET-data gebruikt wordt voor voorspellingen van de pathologische respons.

Het PET-gebaseerde model voor de voorspelling van de pathologische respons, zoals gepresenteerd in Hoofdstuk 3 en 4 van dit proefschrift, werd in Hoofdstuk 5 gevalideerd met behulp van een tweede patiëntgroep voor wie zowel beeldvorming als behandeling identiek heeft plaatsgevonden als de patiënten waarop het model gebaseerd is. Voor de verdere ontwikkeling en klinisch toepassing van dergelijke modellen is validatie noodzakelijk. Voor de patiënt groep waarop het model gebaseerd werd resulteerde een $48 \%$ afname van de maximale FDG opname $\left(S U V_{\max }\right)$ in de tumor in een sensitiviteit en specificiteit van respectievelijk 64 en $100 \%$. Voor de patiënt groep waarmee het model gevalideerd werd resulteerde de $48 \% \mathrm{SUV}_{\max }$ afname in een sensitiviteit van $83 \%$ en een specificiteit van 93\%, met één pathologische nietresponder die vals positief geclassificeerd werd als pathologische responder. 


\section{Deel II: Perfusie-CT beeldvorming gedurende hypogefractioneerde radiotherapie}

In Hoofdstuk 2 werd voor patiënten preoperatief behandeld met hypogefractioneerde RT geen significante verandering van de metabole activiteit van de tumor gevonden na analyse van de herhaalde PET-data. Om voor deze patiëntgroep toch een mogelijke vroege behandelrespons waar te nemen werd in Hoofdstuk 6 van dit proefschrift de perfusie van de tumor bestudeerd met behulp van herhaalde perfusie-CT beeldvorming. Voor dit onderzoek werden 23 NLARC patiënten ge-includeerd, welke perfusieCT onderzoeken ondergingen voorafgaande aan de behandeling en op de dag van de laatste radiotherapie fractie. Perfusie werd gequantificeerd op basis van drie farmacokinetische parameters verkregen met behulp van het Kety-model: $K^{\text {trans }}, v_{e}$ en $v_{p}$. Voor elke patiënt werden perfusie verschillen tussen tumor en spierweefsel en perfusie veranderingen gedurende pre-operatieve behandeling bestudeerd. Voor beide scans, pre- en post-therapie, werden in tumor weefsel hogere gemiddelde perfusie waardes waargenomen in vergelijking met spierweefsel. Gedurende pre-operatieve behandeling met hypogefractioneerde RT werd een significante stijging van de perfusie waargenomen binnen de tumor, terwijl voor spierweefsel constante perfusie-niveaus werden waargenomen.

Om de correlatie tussen tumor perfusie en de FDG opname in rectum tumoren te onderzoeken, werd in Hoofdstuk 7 van dit proefschrift de perfusie en FDG opname in rectum tumoren bestudeerd op intra-tumor niveau zowel voor aanvang van behandeling als op de laatste dag van de behandeling met hypogefractioneerde RT. Op tumor niveau werden de gemiddelde en maximale FDG opname in de tumor (SUV mean, SUVmax) en de perfusie waardes ( $K^{\text {trans }}$ ) gequantificeerd en gecorreleerd. Vervolgens werd de tumor in 8 sub-regio's verdeeld. Ook voor elk van de 8 subregio's werden de gemiddelde en maximale FDG opname en perfusie waardes bepaald en gecorreleerd. Verder werd de gemiddelde FDG opname in de voxels met de $25 \%$ laagste perfusie waardes vergeleken met de FDG opname in de voxels met de $25 \%$ hoogste perfusie waardes. Op tumor niveau werd er zowel voor de gemiddelde als maximale FDG opname en tumor perfusie een positieve correlatie gevonden $(\rho=0.596, p=0.001$ en $\rho=0.779, p<0.001)$. Ook op intra-tumor niveau werden deze correlaties gevonden $(\rho=0.413, p<0.001$ en $\rho=0.540, p<0.001)$. De gemiddelde FDG opname in de voxels met de $25 \%$ hoogste perfusie waardes was significant hoger in vergelijking met de gemiddelde FDG opname in de voxels met de $25 \%$ laagste perfusie waardes $(10.6 \pm 5.1 \%$, $\mathrm{p}=0.017)$.

\section{Deel III: Validatie van geautomatiseerde PET-gebaseerde tumor deli- neatie}

In Hoofdstuk 8 van dit proefschrift werd de automatisch gegenereerde tumor contour, gebaseerd op SUV-iso-contouring, gevalideerd met behulp van het tumor volume bepaald aan de hand van pathologisch onderzoek van het preparaat na pre-operatieve behandeling met hypogefractioneerde RT. 
Automatische PET gebaseerde tumor contouring resulteerde in een gemiddeld tumor volume van $16.2 \pm 11.3 \mathrm{~cm}^{3}\left(7.7\right.$ tot $\left.38.7 \mathrm{~cm}^{3}\right)$, terwijl pathologisch onderzoek van de preparaten een gemiddeld tumor volume van $16.0 \pm 11.5 \mathrm{~cm}^{3}\left(7.8\right.$ tot $\left.36.5 \mathrm{~cm}^{3}\right)$ opleverde, met een onderlinge correlatie coëfficiënt van $0.964(p<0.001)$. Een gemiddeld verschil van $-0.2 \pm 2.0 \mathrm{~cm}^{3}\left(-2.2\right.$ tot $\left.3.0 \mathrm{~cm}^{3}\right)$ werd gevonden wanneer de PET gebaseerde tumor volumes vergeleken werden met de pathologische tumor volumes. De sterke correlatie tussen de twee tumor volumes demonstreert dat automatische tumor contouring gebaseerd op PET-beeldvorming resulteert in een nauwkeurige delineatie van rectum tumoren. 



\section{Dankwoord}


Allereerst wil ik mijn promotor prof. Philippe Lambin en copromotoren dr. ir. Michel Öllers en dr. Guido Lammering bedanken voor hun hulp en bijdrage aan dit onderzoek.

Beste Philippe, geregeld bent $u$ in de KFG projectruimte aanwezig om door de promovendi en onderzoekers bijgepraat te worden over de vorderingen van de projecten waar we aan werken. Ook was $u$ vaak aanwezig bij het tweewekelijkse abdomenoverleg, waar u altijd interessante ideeën aandroeg om het onderzoek verder uit te bereiden en de analyse methodes te perfectioneren.

Guido, ondanks je drukke agenda, kon ik altijd bij je terecht om over de onderzoeksresultaten te discussiëren. De manuscripten die in dit proefschrift zijn opgenomen zijn mede tot stand gekomen door jouw nauwgezette correctie en vaak zelfs herformulering van gehele alinea's. Zeker tijdens het schrijven van mijn eerste manuscripten heb ik veel van je geleerd, waar ik nu, bij het schrijven van artikelen of abstracts voor congressen veel profijt van heb.

Beste Michel, de deur van je kantoor stond altijd voor me open als ik een vraag had of even behoefte had aan een overpeinzing van nieuwe resultaten. Ik heb tijdens mijn promotieonderzoek veel geleerd over de PET-techniek. Buiten je hulp tijdens mijn promotieonderzoek wil ik je ook graag bedanken voor de mountainbike vakanties en weekends. We hebben de afgelopen jaren samen een aantal marathon-wedstrijden gereden in binnen en buitenland. De trainingsritten en de voorbereidingen voor de wedstrijden waren uitstekend geschikt als afleiding van mijn promotie onderzoek.

De promotiecommissie wil ik bedanken voor het lezen en beoordelen van dit proefschrift.

Beste Frank, in 2009 bent u bij MAASTRO Clinic begonnen als hoofd Research binnen de klinische fysica groep. $U$ maakt het mede mogelijk dat er veel onderzoek gedaan kan worden binnen MAASTRO Clinic, waar ik u erg dankbaar voor ben.

Wie ik in dit dankwoord niet mag vergeten zijn de medewerkers van de PET-CT groep. Zonder de inzet van de PET-CT-laboranten was de uitvoering van de onderzoeken onmogelijk geweest. De lange dynamische PET-CT scans, vaak ook nog op 4 tijdstippen, voor, tijdens en na behandeling, vergde veel van jullie tijd en aanpassingsvermogen. Bedankt voor de tips hoe we de protocollen konden aanpassen om de onderzoeken voor de patiënten minder belastend te maken. Ook de ondersteuning, verantwoordelijk voor het inplannen van de PET-CT scans, wil ik bedanken voor de vaak onzichtbare, maar niet minder belangrijke hulp achter de schermen.

Graag zou ik in dit dankwoord mijn dank willen laten uitgaan naar de patiënten die deelgenomen hebben aan de verschillende studies. Zonder hun deelname waren de onderzoeken die in dit proefschrift gepresenteerd worden niet mogelijk geweest. 
Verder wil ik de pathologie afdelingen van het Laurentius Ziekenhuis te Roermond, het Orbis Medisch Centrum Sittard, het Sint Jansgasthuis te Weert, het Atrium Medisch Centrum Heerlen en het Maastricht Universitair Medisch Centrum bedanken voor het opzoeken en opsturen van de pathologieverslagen en de HE-coupes van de patiënten. Meer specifiek wil ik Dr. Robert Riedl bedanken voor het bepalen van de TRG's van de patiënten, waardoor we de PET-gebaseerde metabole respons konden correleren met de pathologische respons. Ook Dr. Marius Nap wil ik graag bedanken voor zijn hulp bij het bepalen van de tumor volumes op basis van de pathologische preparaten.

Beste Jörgen en Jeroen, ook jullie wil ik bedanken voor jullie hulp bij dit proefschrift. Voor de patiënten waarvan ik niet precies wist waar ik op "TrueD" de overgang tussen tumor en blaas moest zien, kon ik bij jullie terecht. Ook voor het intekenen van tumoren, het registreren van verschillende PET-CT datasets of het opvragen van pathologie verslagen kon ik op jullie hulp rekenen.

Hugo en Andre, jullie hebben me begeleid tijdens mijn afstudeerproject voorafgaand aan dit promotieonderzoek en mogen daarom zeker niet ontbreken in dit dankwoord. Mede door jullie begeleiding heb ik tijdens mijn afstudeerproject een fundering kunnen bouwen, waar ik tijdens mijn promotieonderzoek op verder heb kunnen bouwen.

Verder wil ik alle co-auteurs van de manuscripten bedanken voor jullie bijdrage en het publiceerbaar maken van de manuscripten die in dit proefschrift zijn opgenomen.

Ook de rest van de KFG groep, bedankt voor de mooie momenten tijdens de KFGetentjes en -activiteiten en natuurlijk de lunchpauzes. Als het om de KFG lunchpauzes gaat, verdient Sonia zeker een extra woord van dank. Sonia, bedankt voor alle mooie verhalen die je altijd te vertellen had en de mooie discussies die we tijdens de lunch gevoerd hebben! Alle kamergenoten waarmee ik de afgelopen jaren een kamer gedeeld heb, bedankt voor de mooie discussies, al dan niet over werk gerelateerde dingen. Ruud, bedankt voor het ontwerpen van de kaft van mijn proefschrift.

Paranimfen, bedankt voor jullie steun tijdens het afronden van mijn promotie en jullie hulp bij het organiseren van deze dag.

Als laatste wil ik graag mijn familie en vrienden bedanken. Bij m'n vrienden kon ik altijd terecht om na een werkweek te genieten van een (3D) film of een goed gesprek. Mijn ouders wil ik graag bedanken voor de mogelijkheden en vrijheden die ze me altijd geboden hebben om te studeren. Bedankt! Dankzij jullie steun is dit mooie proefschrift ten stand gekomen, terwijl het voor jullie niet altijd duidelijk was wat m'n werk nu eigenlijk precies inhield. Ook de vakanties in de Alpen waren mooie gelegenheden om mijn hoofd weer "leeg te maken", waarna ik weer met frisse motivatie aan de slag kon gaan! 



\section{Curriculum Vitae}


Marco Janssen werd geboren op 24 mei 1984 te Roermond. In 2002 voltooide hij het Atheneum op scholengemeenschap Sint Ursula te Horn. Hij vervolgde zijn studie met de opleiding Biomedische Technologie (BMT) aan de Technische Universiteit te Eindhoven (TU/e). Na de driejarige bachelor-opleiding koos hij voor de tweejarige masteropleiding Medical Engineering (ME), gericht op het verrichten van onderzoek in een klinische omgeving. In het eerste jaar van de masteropleiding voltooide hij een stage bij de afdeling biofysica aan de universiteit van Maastricht (UM) in samenwerking met de afdeling cardiologie van het Maastricht Universitair Medisch Centrum (MUMC+), gericht op atriumfibrillatie bij patiënten met cardiologische problemen. In 2007 rondde hij zijn ingenieursopleiding af met een afstudeer-project bij MAASTRO Clinic te Maastricht, waar hij onderzoek deed naar dynamische PET-CT beeldvorming bij patiënten met endeldarmkanker. Tijdens het voltooien van dit project werd de interesse naar beeldvorming binnen de radiotherapie gewekt. In september 2007 startte hij dan ook promotieonderzoek naar PET-CT beeldvorming bij patiënten met endeldarmkanker binnen MAASTRO Clinic. 


\section{Publications}


Residual metabolic tumor activity after chemo-radiotherapy is mainly located in initially high FDG uptake areas in rectal cancer.

van den Bogaard J, Janssen MHM, Janssens G, Buijsen J, Reniers B, Lambin P, Lammering $\mathrm{G}$, Öllers $M C$

Radiother Oncol 2011; 99(2): 137-141

PET based treatment response evaluation in rectal cancer: prediction and validation. Janssen MHM, Öllers MC, van Stiphout RGPM, Rield RG, van den Bogaard J, Buijsen J, Lambin P, Lammering $\mathrm{G}$

Int J Radiat Oncol Biol Phys 2011; Accepted for publication

Repeated PET-CT and perfusion-CT imaging in rectal cancer: FDG uptake corresponds with tumor perfusion.

Janssen MHM, Aerts HJWL, Buijsen J, Lambin P, Lammering G, Öllers MC Int J Radiat Oncol Biol Phys 2011; Accepted for publication

FDG-PET provides the best correlation with the tumor specimen compared to MRI and $\mathrm{CT}$ in rectal cancer.

Buijsen J, van den Bogaard J, Janssen MHM, Bakers FC, Engelsman S, Öllers MC, BeetsTan RGH, Nap M, Beets GL, Lambin P, Lammering G

Radiother Oncol 2011; 98(2): 270-276

Development and external validation of a predictive model for pathological complete response of rectal cancer patients including sequential PET-CT imaging.

van Stiphout RGPM, Lammering G, Buijsen J, Janssen MHM, Gambacorta MA, Slagmolen $P$, Lambrecht $M$, Rubello D, Gava M, Giordano A, Postma EO, Haustermans K, Capirci C, Valentini V, Lambin P

Radiother Oncol 2011; 98(1): 126-133

PET imaging of hypoxia using [18F]HX4: a phase I trial.

van Loon J, Janssen MHM, Öllers MC, Aerts HJWL, Dubois L, Hochstenbag M, Dingemans AM, Lalisang R, Brans B, Windhorst B, van Dongen GA, Kolb $H$, Zhang J, de Ruysscher D, Lambin $\mathrm{P}$

Eur J Nucl Med Mol Imaging 2010; 37(9): 1663-1668

Blood glucose level normalization and accurate timing improves the accuracy of PETbased treatment response predictions in rectal cancer.

Janssen MHM, Öllers MC, van Stiphout RGPM, Riedl RG, van den Bogaard J, Buijsen J, Lambin P, Lammering $\mathrm{G}$

Radiother Oncol 2010; 95(2): 203-208 
Evaluation of early metabolic responses in rectal cancer during combined radiochemotherapy or radiotherapy alone: sequential FDG-PET-CT findings.

Janssen MHM, Ollers MC, van Stiphout RGPM, Buijsen J, van den Bogaard J, de Ruysscher D, Lambin P, Lammering $\mathrm{G}$

Radiother Oncol 2010; 94(2): 151-155

Tumor perfusion increases during hypofractionated short-course radiotherapy in rectal cancer: sequential perfusion-CT findings.

Janssen MHM, Aerts HJWL, Kierkels RGJ, Backes WH, Ollers MC, Buijsen J, Lambin P, Lammering $\mathrm{G}$

Radiother Oncol 2010; 94(2): 156-160

Comparison between perfusion computed tomography and dynamic contrastenhanced magnetic resonance imaging in rectal cancer.

Kierkels RGJ, Backes WH, Janssen MHM, Buijsen J, Beets-Tan RGH, Lambin P, Lammering $\mathrm{G}$, Ollers MC, Aerts HJWL

Int J Radiat Oncol Biol Phys 2010; 77(2): 400-408

Accurate prediction of pathological rectal tumor response after two weeks of preoperative radiochemotherapy using (18)F-fluorodeoxyglucose-positron emission tomography-computed tomography imaging.

Janssen MHM, Ollers MC, Riedl RG, van den Bogaard J, Buijsen J, van Stiphout RGPM, Aerts HJWL, Lammering $\mathrm{G}$

Int J Radiat Oncol Biol Phys 2010; 77(2): 392-399

Tumor delineation based on time-activity curve differences assessed with dynamic fluorodeoxyglucose positron emission tomography-computed tomography in rectal cancer.

Janssen MHM, Aerts HJWL, Ollers MC, Bosmans G, Lee JA, Buijsen J, de Ruysscher D, Lambin P, Lammering G, Dekker ALAJ

Int J Radiat Oncol Biol Phys 2009; 73(2): 456-465 



\section{List of abbreviations}




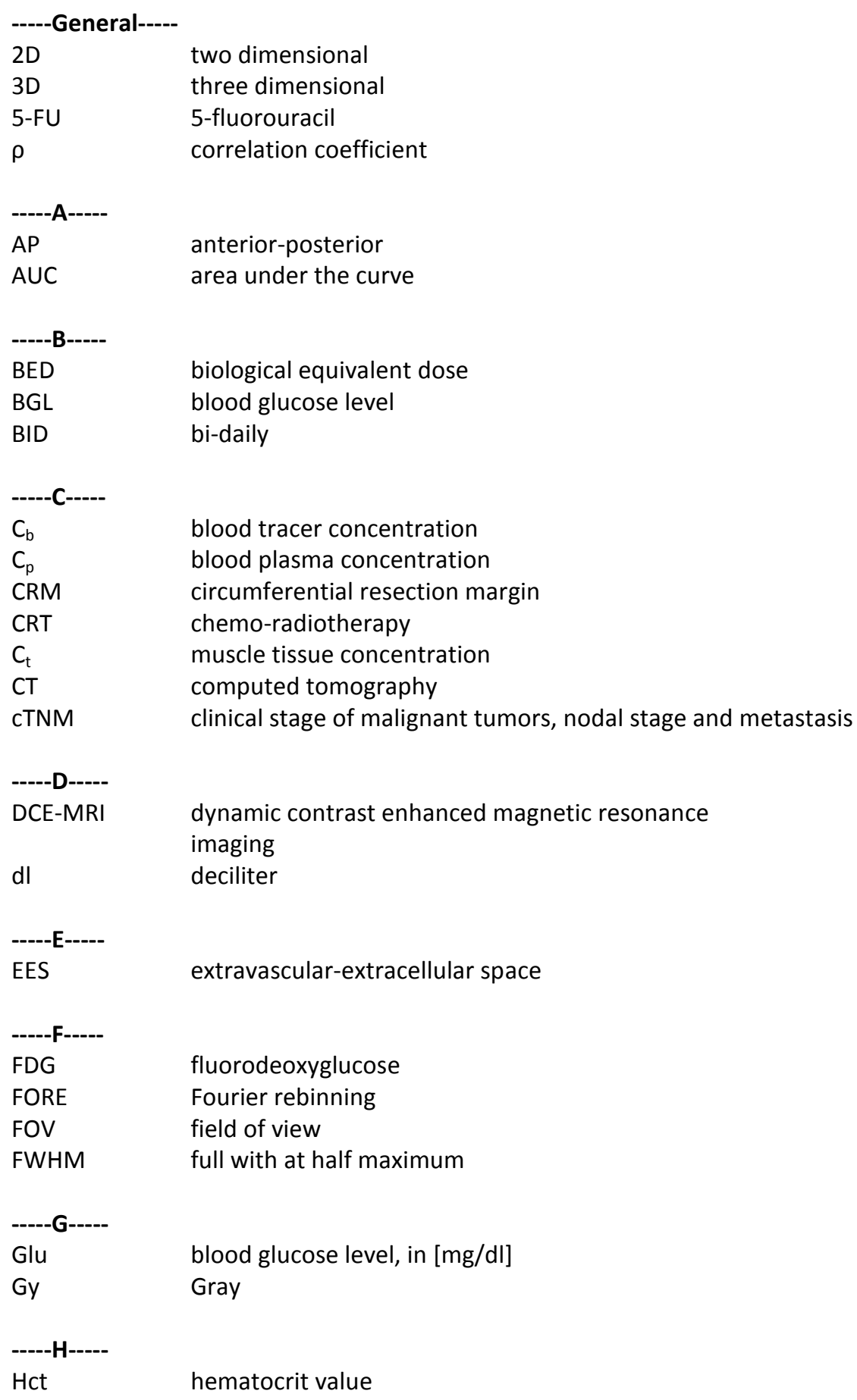


-----I-----

ICRU

-----K-----

$\mathrm{kg}$

$\mathrm{K}^{\text {trans }}$

$k V p$

-----L-----

LARC

LM

-----M-----

$\mathrm{MBq}$

$\mathrm{mCR}$

$\mathrm{mg}$

$\mathrm{mm}$

min.

$\mathrm{ml}$

MRF

$M R(I)$

MV

$\mathrm{m}^{2}$

-----N-----

NLARC

-----O-----

OSEM

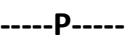

PA

PCR

PCT

PET

p.i.

pPR

PVE

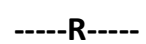

RI

ROC

ROI

RT
International Commission on Radiation Units \&

Measurements

kilogram

transfer rate of the contrast agent from the blood plasma into the EES

kilo-voltage peak (energy)

locally advanced rectal cancer

list-mode

mega Becquerel

metabolic complete responder

milligram

millimeter

minute

milliliter

mesorectal fascia

magnetic resonance (imaging)

megavolt

square meter

non-locally advanced rectal cancer

two dimensional ordered subsets expectation

maximization

posterior-anterior

pathological complete response / responder

perfusion-CT

positron emission tomography

post injection

pathological partial response / responder

partial volume effect

response index

receiver operating characteristics

region of interest

radiotherapy 
156 | List of abbreviations

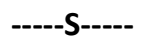

$\mathrm{S}$

SBR

SCRT

SD

SNR

SUV

-----T-----

TAC

TEM

TNM

TME

TRG

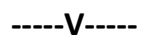

$\mathrm{v}_{\mathrm{e}}$

VOI

$v_{p}$

-----Y-----

урт second

signal to background ratio

(hypofractionated) short course radiotherapy

standard deviation

signal to noise ratio

standardized uptake value

time activity curve

transanal endoscopic micro-surgery

tumor-nodal-metastasis classification of malignancies

total mesorectal excision

tumor regression grade

fractional volume of the EES

volume of interest

fractional blood plasma volume

pathological stage of malignant tumors 